\title{
CORE-LOADING DIAGRAMS FOR EBR-II RUNS 39 THROUGH 50 \\ (December 1969 through mid-August 1971)
}

Compiled by

J. C. Case

\author{
EBR-II Project \\ Argonne National Laboratory \\ Argonne, Illinois - Idaho Falls, Idaho
}

NOTICE-

This report was prepared as an account of work sponsored by the United States Government. Neither the United States nor the United States Atomic Energy Commission, nor any of their employees, nor any of their contractors, subcontractors, or their employees, makes any warranty, express or implied, or assumes any legal liability or responsibility for the accuracy, completeness or usefulness of any information, apparatus, product or process disclosed, or represents that its use would not infringe privately owned rights.

Work performed under the ausipces of the U. S. Atomic Energy Commission 


\section{DISCLAIMER}

This report was prepared as an account of work sponsored by an agency of the United States Government. Neither the United States Government nor any agency Thereof, nor any of their employees, makes any warranty, express or implied, or assumes any legal liability or responsibility for the accuracy, completeness, or usefulness of any information, apparatus, product, or process disclosed, or represents that its use would not infringe privately owned rights. Reference herein to any specific commercial product, process, or service by trade name, trademark, manufacturer, or otherwise does not necessarily constitute or imply its endorsement, recommendation, or favoring by the United States Government or any agency thereof. The views and opinions of authors expressed herein do not necessarily state or reflect those of the United States Government or any agency thereof. 


\section{DISCLAIMER}

Portions of this document may be illegible in electronic image products. Images are produced from the best available original document. 
$-2-$

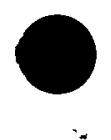

4

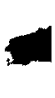

$r$ 
CORE-LOADING DIAGRAMS FOR EBR-II RINS 39 THROUGH 50

(December 1969 through mid-August 1971)

Compiled by

J. C. Case

\section{INTRODUCTION}

This report contains the core-loading diagrams for all runs (Nos. 39 through 50) made by EBR-II between December 1969 and mid-August 1971, a period of time during which the reactor accumulated 16,823 MWd (therma1) of operation. (The reactor had been operated for an additional 30,040 MWd between August 1964 and December 1969.) This report supplements a similar report, ANL/EBR-007, which showed the core-loading diagrams for this earlier period.

The loading diagrams appear in numerical, and thus chronological, order. Each diagram is on a righthand page and is faced with a tabulation of additional information pertaining to the run, e.g., core size, dates on which the run started and ended, power generated during the run, and the number and general types of subassemblies that were in the core during the run. At the bottom of each page facing a diagram is a key to the symbols used for the types of subassemblies shown in the diagram.

Locations of the experimental subassemblies are highlighted in each diagram through the use of a black background behind the identifying numbers of the subassemblies. No other subassemblies are identified by number. The center of the core is identified in each diagram by a circle, and the core sectors are identified by the letters $A$ through $F$ around the perimeter of the core diagram.

A table at the end of the report summarizes the pertinent information for every EBR-II run through run 50 . 
RUN 39A

(91-Subassembly Core Size)

Chronology

Date Started ............. 12/16/69

Date Ended ............... . . 1/10/70

Number of Days for Run . . . . . . . 25

Power Data

Cumulative MWd(t) at Start of Run . . . 30,040

Cumulative MWd(t) at End of Run . . . . 30,811

MWd ( $t$ ) Generated During Run . . . . . . 771

Types of Subassemblies in Core

Blanket . . . . . . . . . . . . 25

Core................ . 32

Control Rod ............... 10

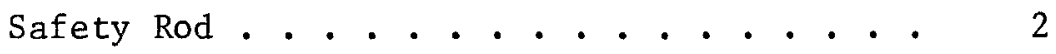

Experimentals (Rows 1-6) . . . . . . . 21

Experimental Subassemblies in Row 7 . . . . . 8

Key to Types of Subassemblies Shown in Loading Diagram

B -- Depleted Uranium

BETH -- Beryllium Thimble

C -- Control Rod

CF -- Controlled-flow Subassembly

D -- Driver Fuel

MK-II -- Mark-II Fuel

Ni -- Nicke1-corrosion Subassembly

P -- 1/2 Driver Fuel - 1/2 Stainless Stee1

S -- Safety Rod

SSCR -- Stainless Steel Control Rod

SSTH -- Stainless Steel Thimble

$\mathrm{X}$-- Experimental Subassembly 


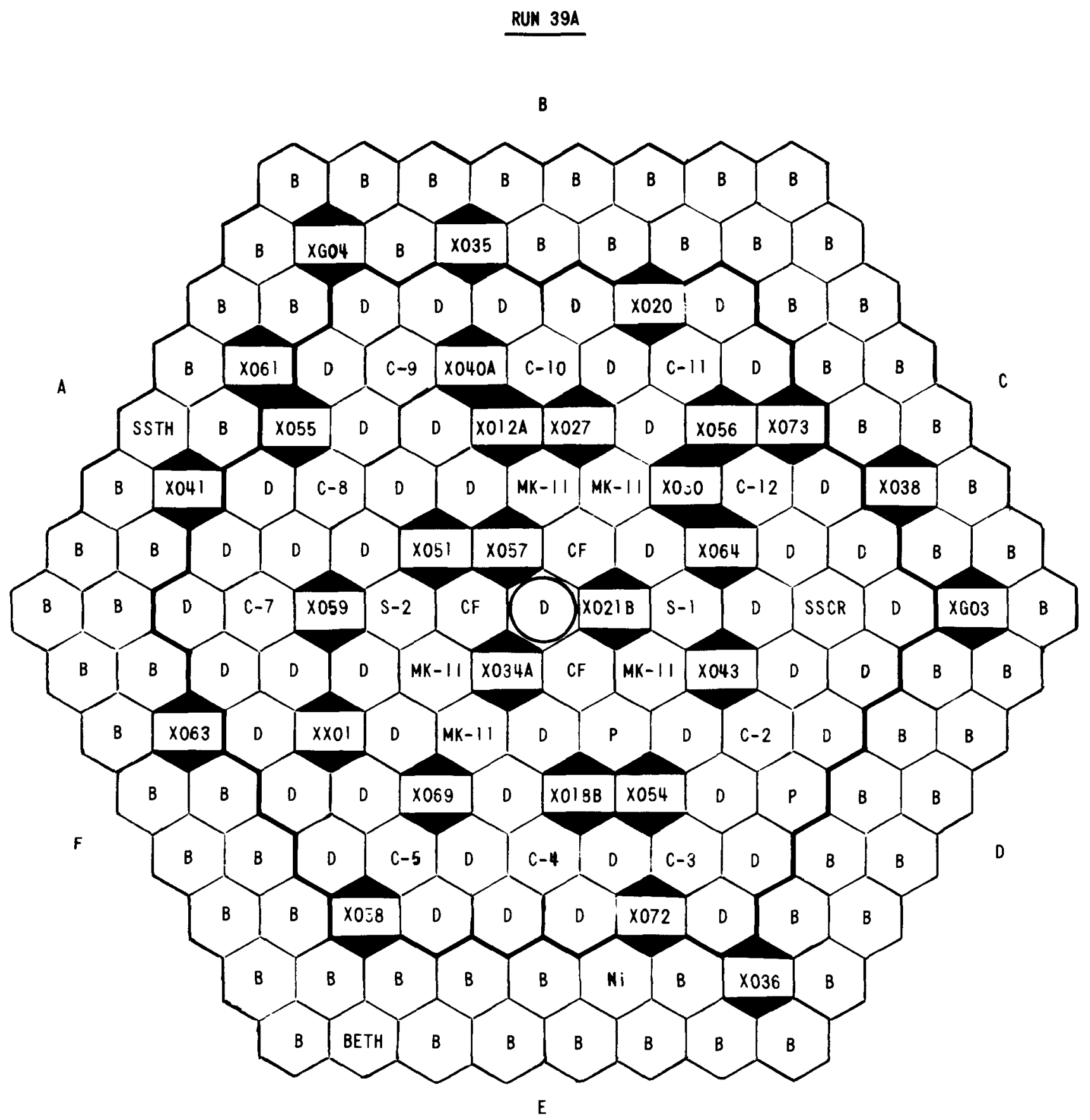


$\underline{\text { RUN } 39 B}$

(90-Subassembly Core Size)

Chronology

Date Started .............. 1/14/70

Date Ended .............. 1/19/70

Number of Days for Run . . . . . . . . 5

Power Data

Cumulative MWd(t) at Start of Run . . . . 30,811

Cumulative MWd(t) at End of Run . . . . 30,996

MWd $(t)$ Generated During Run . . . . . . 185

Types of Subassemblies in Core

Blanket ........... . . 23

Core ................ . 30

Control Rod .............. 10

Safety Rod . . . . . . . . . . . 2

Experimentals (Rows 1-6). . . . . . . 24

Experimenta1 Subassemblies in Row 7 . . . . . 8

Key to Types of Subassemblies Shown in Loading Diagram

$$
\begin{aligned}
& \text { B -- Depleted Uranium } \\
& \text { BETH -- Beryllium Thimble } \\
& \text { C -- Control Rod } \\
& \text { CF -- Controlled-flow } \\
& \text { D -- Driver Fue1 } \\
& \text { MK-II -- Mark-II Fue1 } \\
& \text { Ni -- Nickel-corrosion Subassembly } \\
& \text { S -- Safety Rod } \\
& 70 \% \text {-- Driver Fue1 Enriched to } 70 \%{ }^{235} \mathrm{U} \\
& \text { SSCR -- Stainless Steel Control Rod } \\
& \text { SSTH -- Stainless Steel Thimble } \\
& \mathrm{X} \text {-- Experimental Subassembly }
\end{aligned}
$$




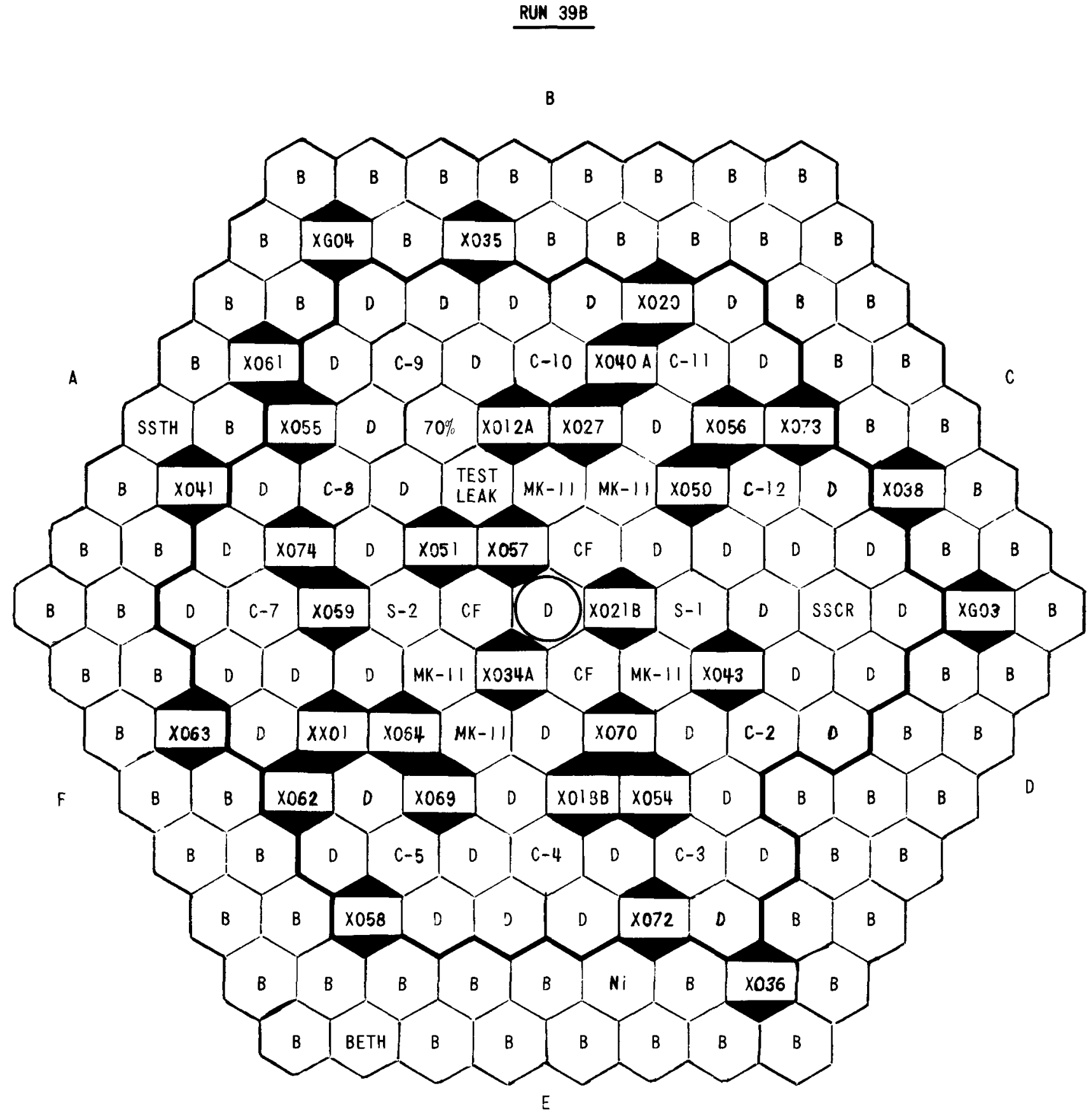


$\underline{\text { RUN 39C }}$

(90-Subassembly Core Size)

Chronology

Date Started ............. 1 1/19/70

Date Ended .............. . 1/28/70

Number of Days for Run . . . . . . . . 9

Power Data

Cumulative MWd(t) at Start of Run . . . 30,996

Cumulative MWd $(t)$ at End of Run . . . . . 31,411

MWd $(t)$ Generated During Run ....... 415

Types of Subassemblies in Core

Blanket . . . . . . . . . . 23

Core . . . . . . . . . . . 30

Control Rod .............. 10

Safety Rod .............. 2

Experimentals (Rows 1-6) . . . . . . 24

Experimental Subassemblies in Row 7 . . . . . 8

Key to Types of Subassemblies Shown in Loading Diagram

B -- Depleted Uranium

BETH -- Beryllium Thimble

C -- Control Rod

CF -- Controlled-flow Subassembly

D -- Driver Fuel

MK-II -- Mark-II Fue1

Ni -- Nickel-corrosion Subassembly

S -- Safety Rod

$70 \%$-- Driver Fuel Enriched to $70 \%{ }^{235_{U}}$

SSCR -- Stainless Steel Control Rod

SSTH -- Stainless Steel Thimble

$\mathrm{X}$-- Experimenta1 Subassembly 


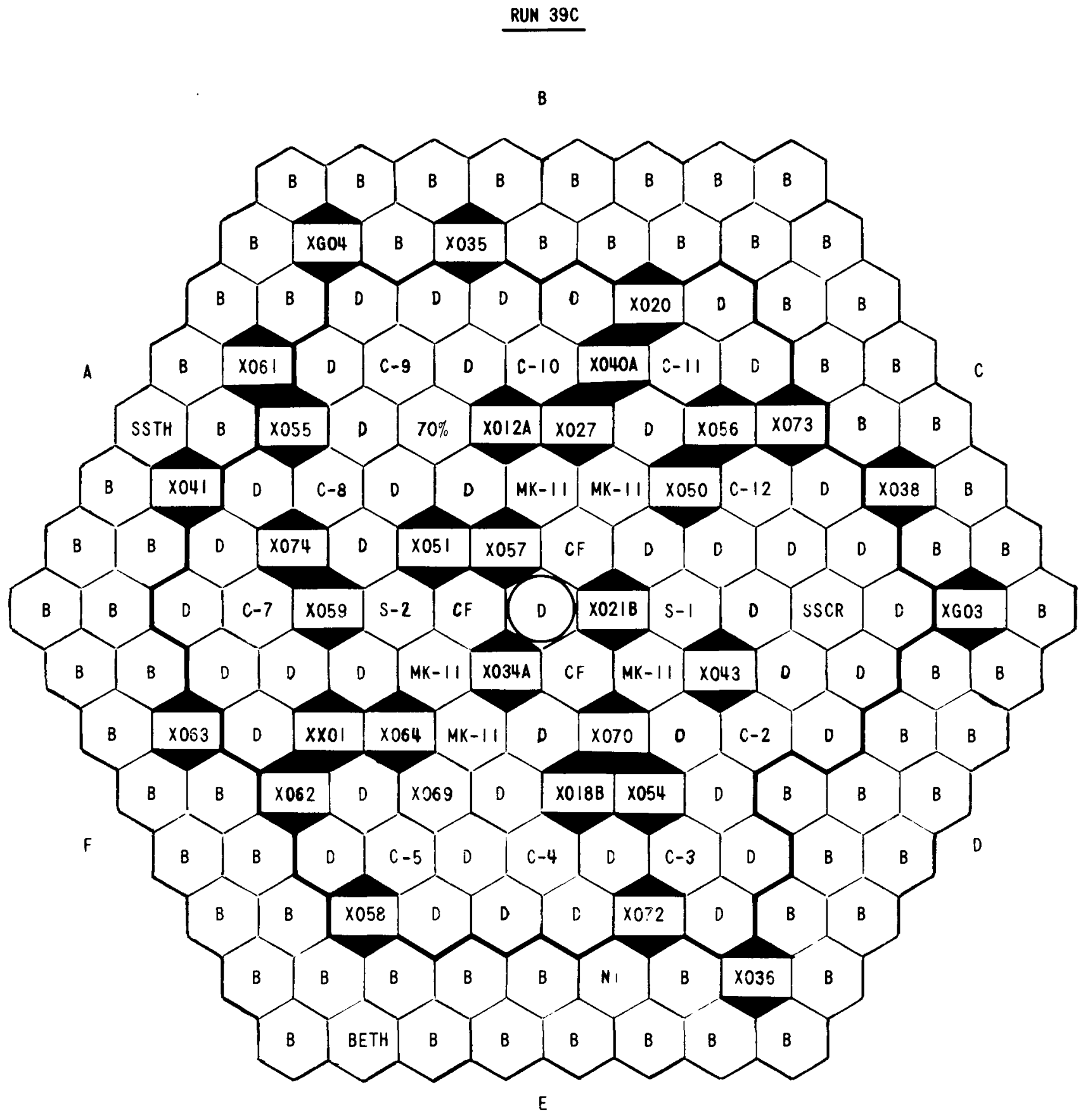


RUN 40A

(91-Subassemb1y Core Size)

Chronology

Date Started ............. $1 / 31 / 70$

Date Ended .............. $2 / 10 / 70$

Number of Days for Run . . . . . . . 10

\section{Power Data}

Cumulative MWd(t) at Start of Run . . . . 31,411

Cumulative MWd $(t)$ at End of Run . . . . 31,854

MWd ( $t$ ) Generated During Run . . . . . . 443

\section{Types of Subassemblies in Core}

Blanket ............ . 26

Core ................. . 27

Control Rod .............. 10

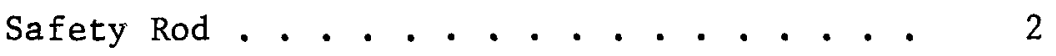

Experimentals (Rows 1-6)........ . 25

Experimental Subassemblies in Row 7 . . . . . 10

Key to Types of Subassemblies Shown in Loading Diagram

B -- Depleted Uranium

BETH -- Beryllium Thimble

C -- Control Rod

CF -- Controlled-flow Subassembly

D -- Driver Fuel

MK-II -- Mark-II Fue1

Ni -- Nickel-corrosion Subassembly

S -- Safety Rod

SSCR -- Stainless Steel Control Rod

SSTH -- Stainless Steel Thimble

$X$-- Experimental Subassembly 


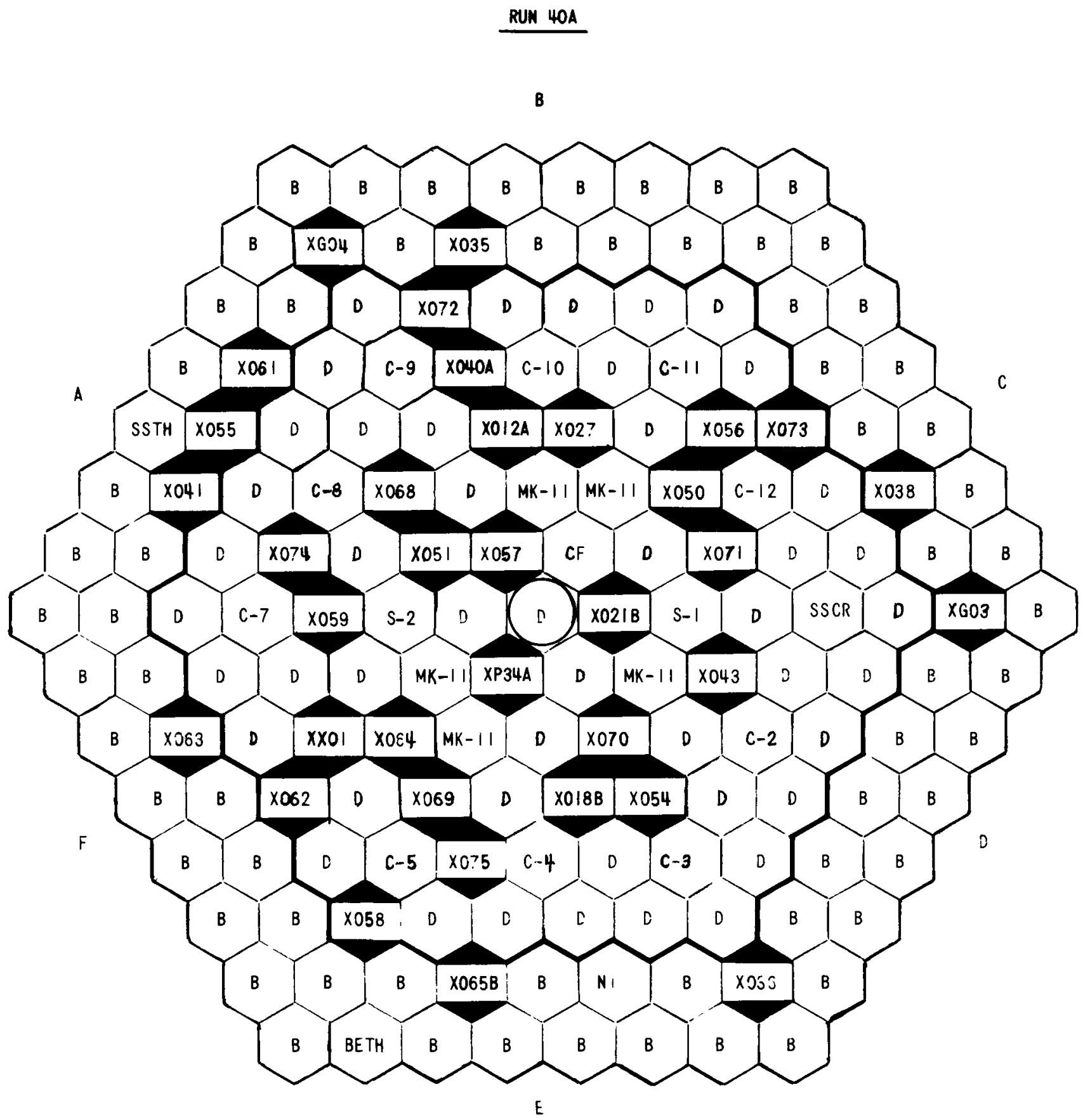


RUN 40B

(91-Subassembly Core Size)

Chronology

Date Started.............. $2 / 10 / 70$

Date Ended............ $3 / 1 / 70$

Number of Days for Run . . . . . . . 19

Power Data

Cumulative MWd(t) at Start of Run . . . . 31,854

Cumulative MWd( $t$ ) at End of Run . . . . 32,778

MWd $(t)$ Generated During Run . . . . . . 924

\section{Types of Subassemblies in Core}

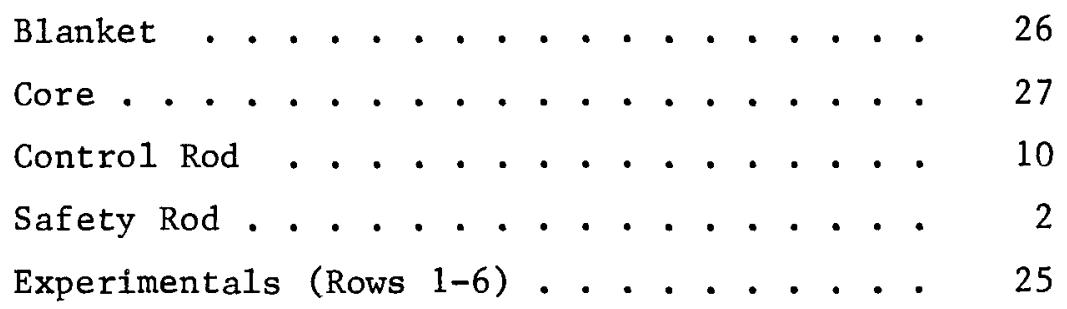

Experimental Subassemblies in Row 7 . . . . 10

Key to Types of Subassemblies Shown in Loading Diagram

$$
\begin{aligned}
& \text { B -- Depleted Uranium } \\
& \text { BETH -- Beryllium Thimble } \\
& \text { C -- Control Rod } \\
& \text { CF -- Controlled-flow Subassembly } \\
& \text { D -- Driver Fuel } \\
& \text { MK-II -- Mark-II Fuel } \\
& \mathrm{Ni} \text {-- Nickel-corrosion Subassembly } \\
& \text { S -- Safety Rod } \\
& \text { SSCR -- Stainless Steel Control Rod } \\
& \text { SSTH -- Stainless Steel Thimble } \\
& \text { X -- Experimental Subassembly }
\end{aligned}
$$




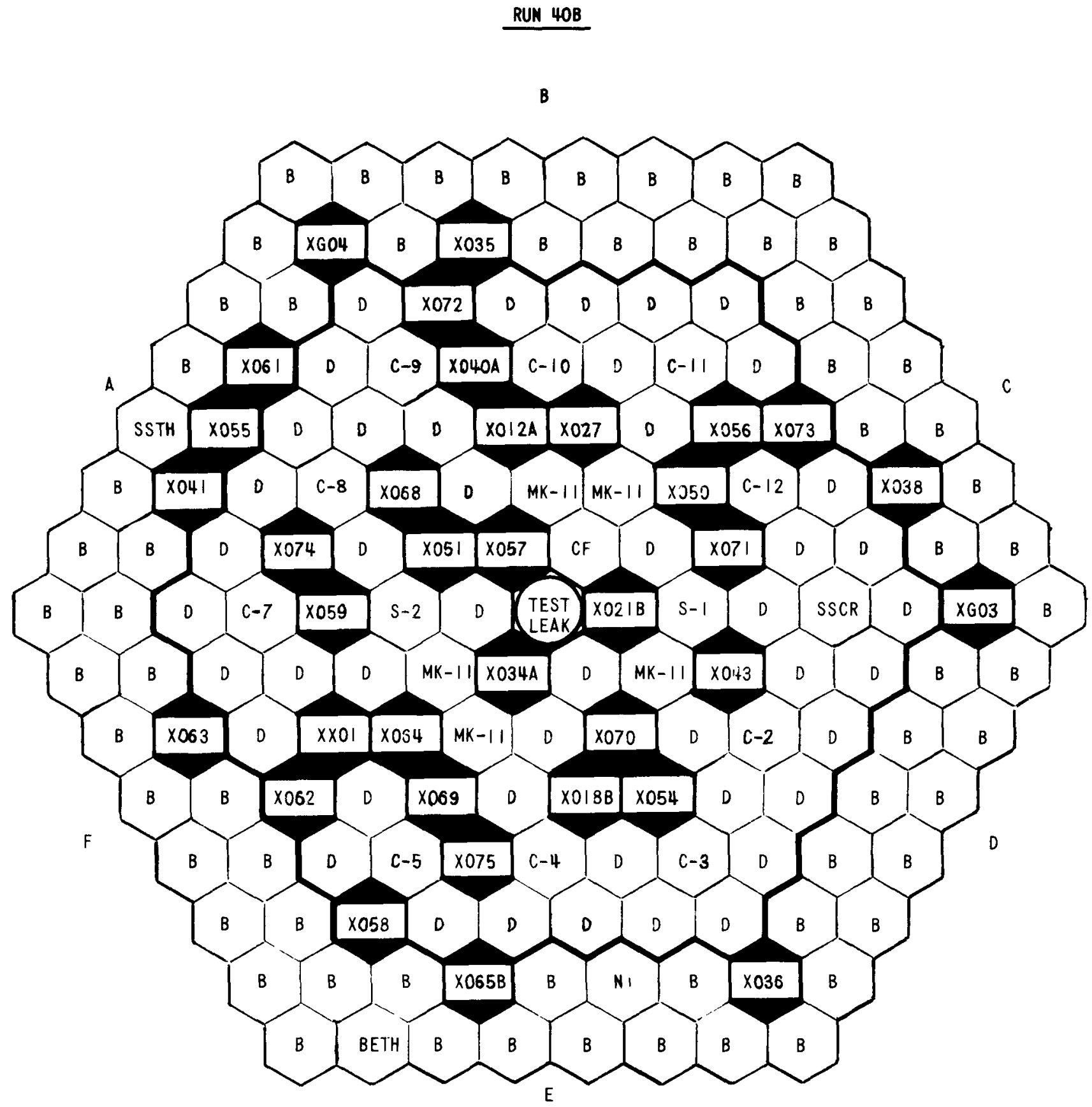


RUN 41A

(91-Subassembly Core Size)

Chronology

Date Started .............. 3/5/70

Date Ended .............. . . 3/25/70

Number of Days for Run ......... . 20

\section{Power Data}

Cumulative MWd(t) at Start of Run . . . . 32,778

Cumulative MWd( $t$ ) at End of Run . . . . 33,739

MWd ( $t$ ) Generated During Run . . . . . . 961

Types of Subassemblies in Core

Blanket . . . . . . . . . . 26

Core . . . . . . . . . . . . 28

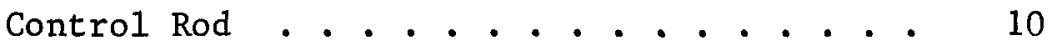

Safety Rod ............... 2

Experimentals (Rows 1-6).......... 23

Experimental Subassemblies in Row 7 . . . . 9

Key to Types of Subassemblies Shown in Loading Diagram

B -- Depleted Uranium

BETH -- Beryllium Thimble

C -- Contro1 Rod

CF -- Controlled-flow Subassembly

D -- Driver Fue1

MK-II -- Mark-II Fue1

$\mathrm{Ni}$-- Nicke1-corrosion Subassembly

P -- 1/2 Driver Fuel - 1/2 Stainless Steel

S -- Safety Rod

SSCR -- Stainless Steel Control Rod

SSTH -- Stainless Steel Thimble

$\mathrm{X}$-- Experimental Subassembly 


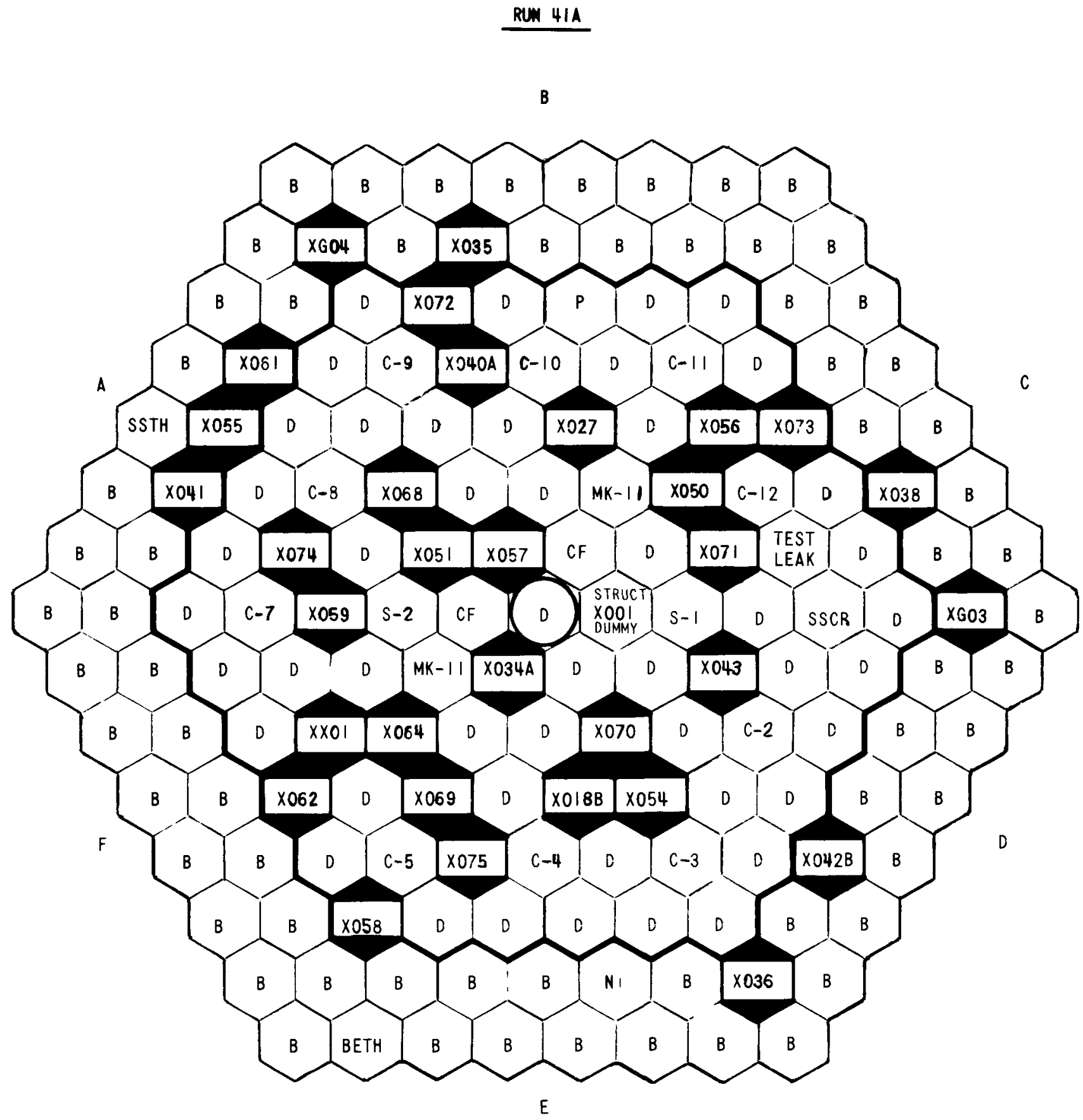


$\underline{\text { RUN } 41 B}$

(91-Subassembly Core Size)

Chronology

Date Started ............. 3/27/70

Date Ended . . . . . . . . . . . 4/1/70

Number of Days for Run . . . . . . . . 5

Power Data

Cumulative MWd(t) at Start of Run . . . . 33,739

Cumulative MWd(t) at End of Run .... . 33,896

MWd( $t$ ) Generated During Run . . . . . . 157

Types of Subassemblies in Core

Blanket . . . . . . . . . 26

Core . . . . . . . . . . . . 28

Control Rod ............. 10

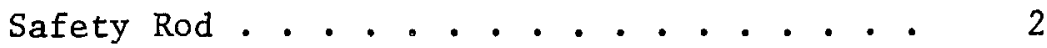

Experimentals (Rows 1-6)......... 23

Experimental Subassemblies in Row 7 . . . . . 10

Key to Types of Subassemblies Shown in Loading Diagram

B -- Depleted Uranium

BETH -- Beryllium Thimble

C -- Control Rod

CF -- Controlled-flow Subassembly

D -- Driver Fuel

MK-II -- Mark-II Fuel

$\mathrm{Ni}$-- Nickel-corrosion Subassembly

P -- 1/2 Driver Fuel - 1/2 Stainless Steel

S -- Safety Rod

SSCR -- Stainless Steel Control Rod

SSTH -- Stainless Steel Thimble

X -- Experimental Subassembly 


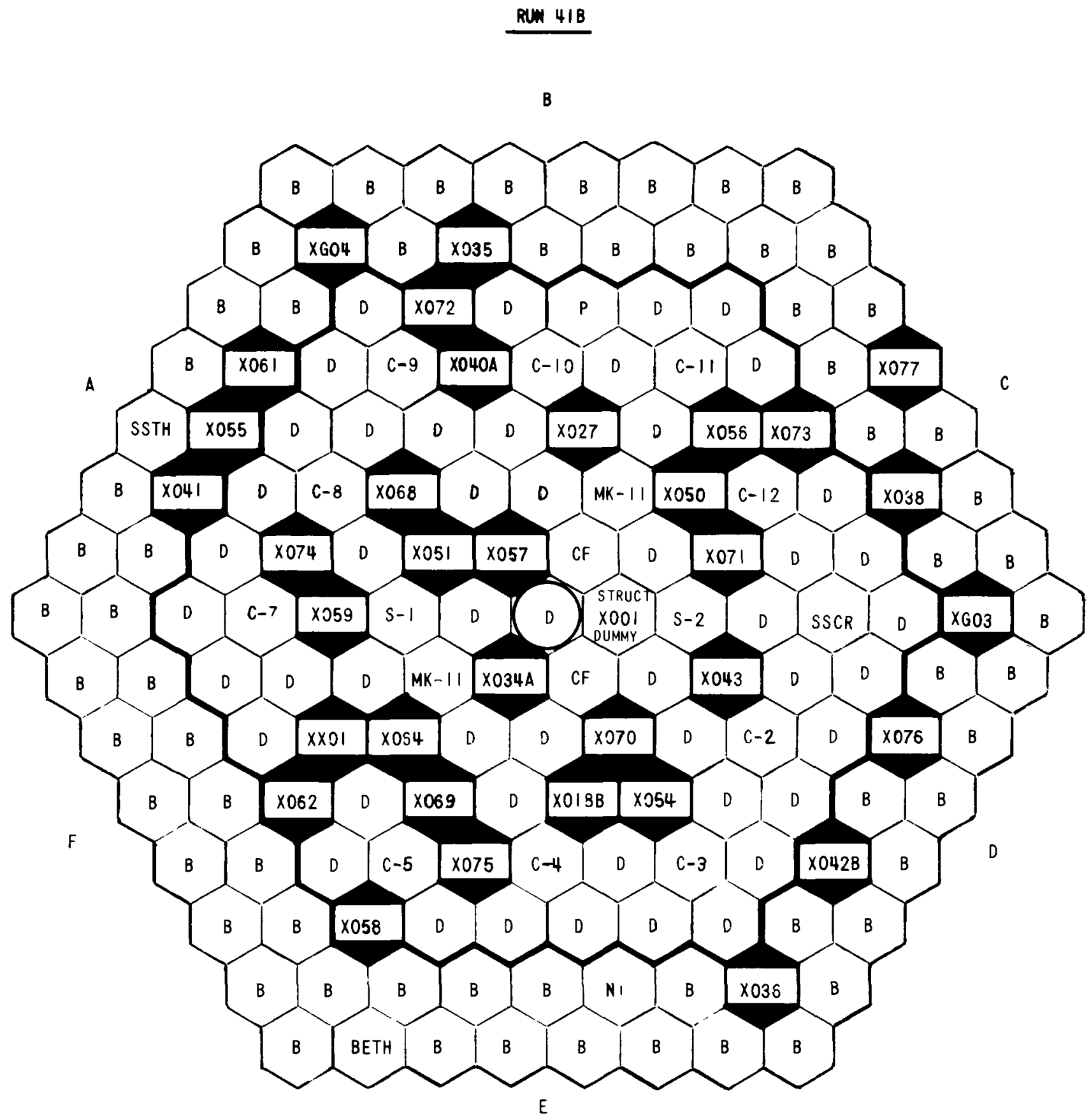


RUN 42

(91-Subassembly Core Size)

Chronology

Date Started ............. 4/18/70

Date Ended ............. 5/18/70

Number of Days for Run .. . . . . . 30

\section{Power Data}

Cumulative MWd(t) at Start of Run . . . 33,896

Cumulative MWd $(t)$ at End of Run . . . . 35,240

MWd $(t)$ Generated During Run . . . . . 1, 1,344

Types of Subassemblies in Core

Blanket . . . . . . . . . 26

Core . . . . . . . . . . . 26

Control Rod ............. . 10

Safety Rod . . . . . . . . . . . 2

Experimentals (Rows 1-6)........ 26

Experimental Subassemblies in Row 7..... 11

Key to Types of Subassemblies Shown in Loading Diagram

$$
\begin{aligned}
& \text { B }- \text { - Depleted Uranium } \\
& \text { BETH }- \text { - Beryllium Thimble } \\
& \text { C }- \text { - Control Rod } \\
& \text { CF }- \text { - Controlled-flow Subassembly } \\
& \text { D }- \text { Driver Fuel } \\
& \text { MK-II }- \text { Mark-II Fuel } \\
& \text { S }- \text { - Safety Rod } \\
& \text { SSCR }-- \text { Stainless Steel Control Rod } \\
& \text { SSTH }-- \text { Stainless Steel Thimble } \\
& \text { X -- Experimental Subassembly }
\end{aligned}
$$




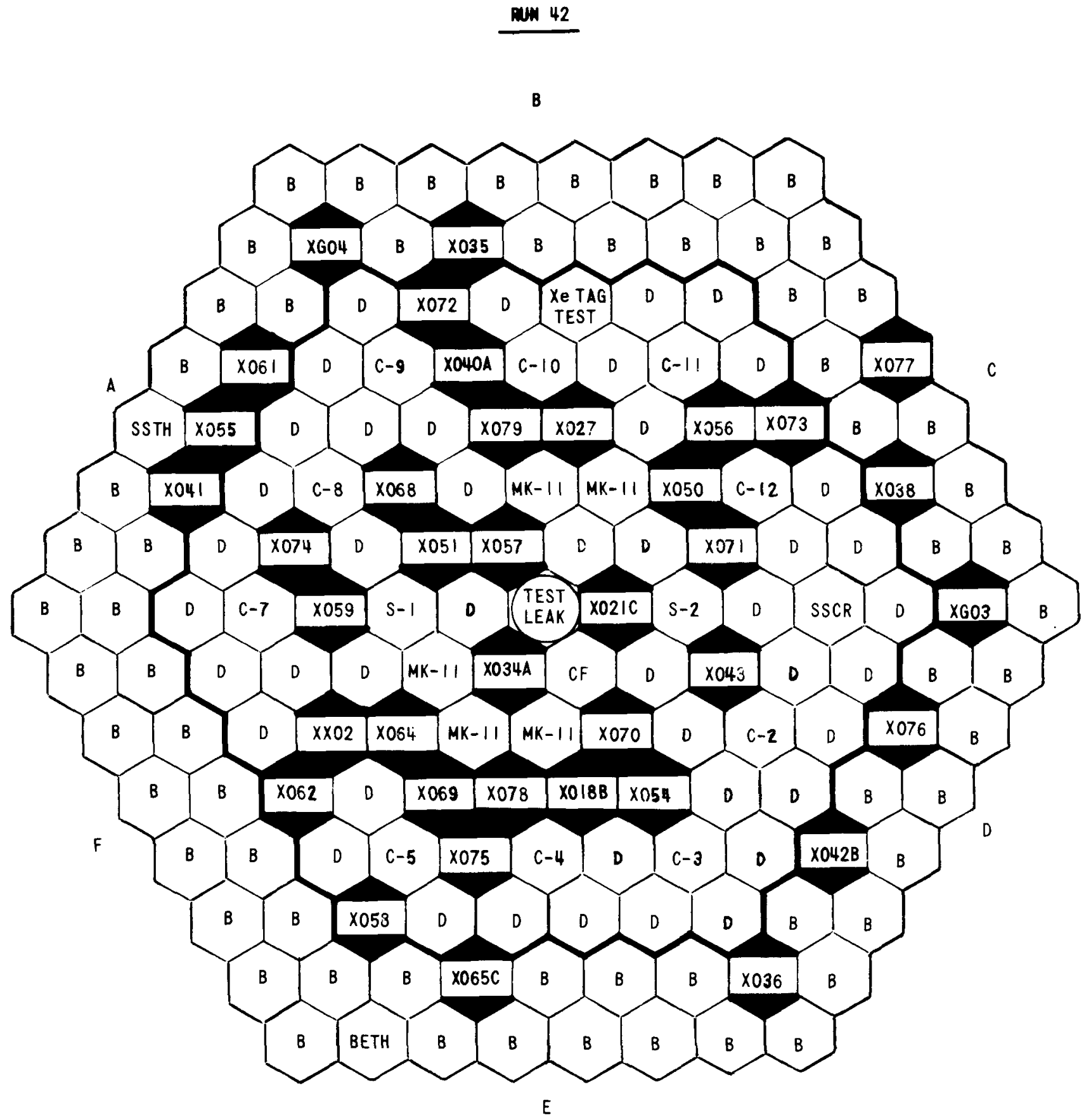


RUN 43

(91-Subassembly Core Size)

Chronology

Date Started ............. 5/26/70

Date Ended ............. 6/23/70

Number of Days for Run . . . . . . . 28

Power Data

Cumulative MWd(t) at Start of Run . . . . 35,240

Cumulative MWd(t) at End of Run . . . . 36,590

MWd $(t)$ Generated During Run . . . . . . 1,350

Types of Subassemblies in Core

Blanket . . . . . . . . . . 25

Core . . . . . . . . . . . . 29

Control Rod ............. 10

Safety $\operatorname{Rod}$. . . . . . . . . . . 2

Experimentals (Rows 1-6)........ 24

Experimental Subassemblies in Row 7 . . . . 10

Key to Types of Subassemblies Shown in Loading Diagram

B -- Depleted Uranium

BETH -- Beryllium Thimble

C -- Control Rod

D -- Driver Fue1

MK-II -- Mark-II Fuel

P -- 1/2 Driver Fue1 - 1/2 Stainless Stee1

$S$-- Safety Rod

SSCR -- Stainless Steel Control Rod

SSTH -- Stainless Steel Thimble

$\mathrm{X}$-- Experimental Subassembly 


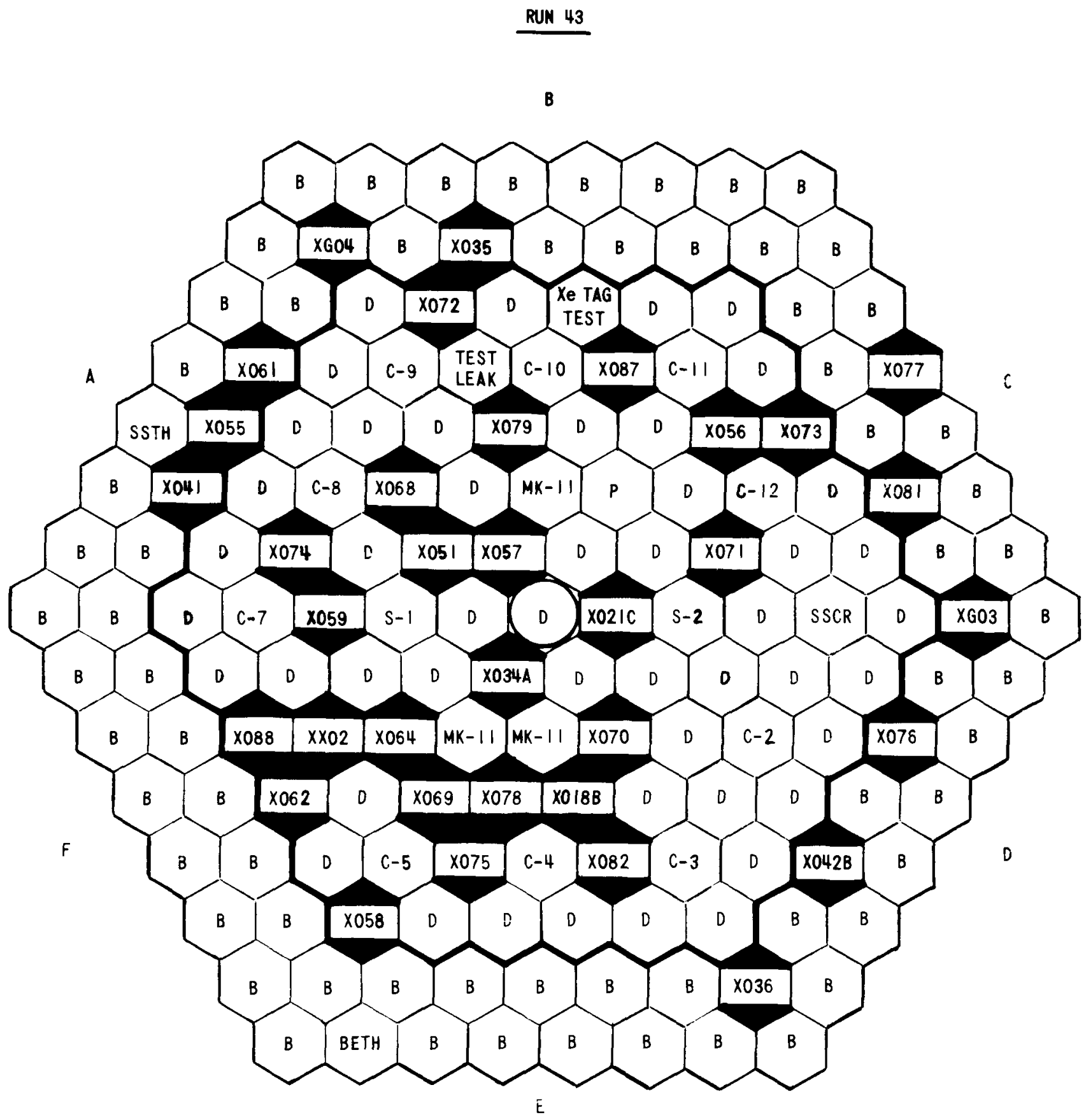


RUN 44A

(91-Subassembly Core Size)

Chronology

Date Started . . . . . . . . . .

$6 / 26 / 70$

Date Ended . . . . . . . . . . .

$7 / 15 / 70$

Number of Days for Run . . . . . . .

Power Data

Cumulative MWd(t) at Start of Run . . . 36,590

Cumulative MWd(t) at End of Run ... . 37,490

MWd $(t)$ Generated During Run ...... 900

Types of Subassemblies in Core

Blanket ............ . . 24

Core .............. . . 30

Contro1 Rod . . . . . . . . . 10

Safety Rod . . . . . . . . . . 2

Experimentals (Rows 1-6) . . . . . . 24

Experimental Subassemblies in Row 7 . . . . 9

Key to Types of Subassemblies Shown in Loading Diagram

B -- Depleted Uranium

BETH -- Beryllium Thimble

C -- Contro1 Rod

CF -- Controlled-flow Subassembly

D -- Driver Fuel

MK-II -- Mark-II Fue1

P - 1/2 Driver Fuel - 1/2 Stainless Steel

S -- Safety Rod

SSCR -- Stainless Steel Control Rod

SSTH -- Stainless Steel Thimble

$X$-- Experimental Subassembly 
RUN 44A

B

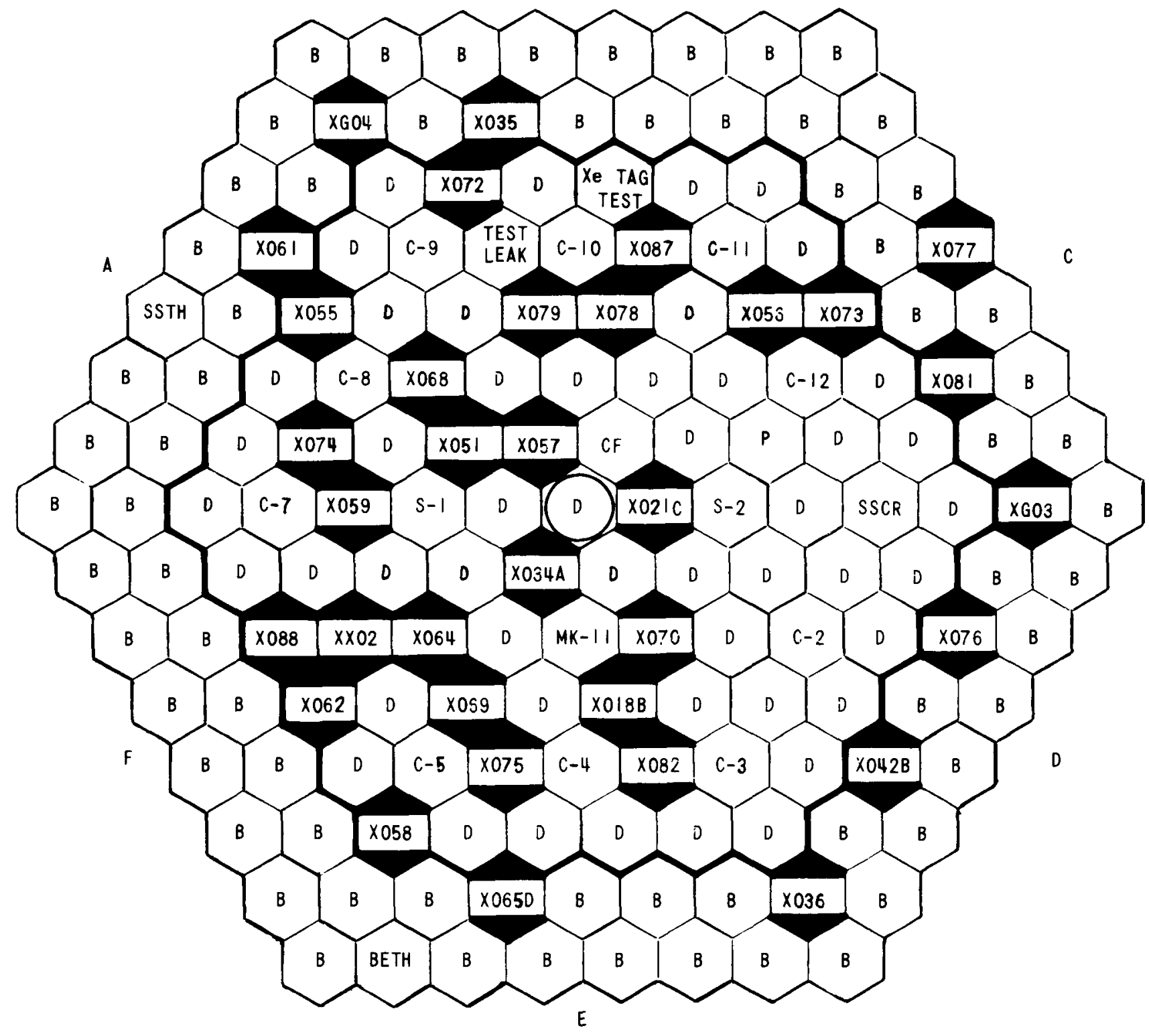


RUN 44B

(91-Subassemb1y Core Size)

Chronology

Date Started ............ $7 / 25 / 70$

Date Ended . . . . . . . . . . . 8/4/70

Number of Days for Run . . . . . . . . 10

Power Data

Cumulative MWd(t) at Start of Run . . . . 37,490

Cumulative MWd $(t)$ at End of Run . . . . 37,941

MWd $(t)$ Generated During Run . . . . . . 451

Types of Subassemblies in Core

Blanket . . . . . . . . . . 24

Core . . . . . . . . . . . 32

Control $\operatorname{Rod} . . . . . . . . . .1010$

Safety $\operatorname{Rod} . . . . . . . . . . . .22$

Experimentals (Rows 1-6) . . . . . . 22

Experimental Subassemblies in Row 7 . . . . . 9

Key to Types of Subassemblies Shown in Loading Diagram

B -- Depleted Uranium

BETH -- Beryllium Thimble

C -- Control Rod

D -- Driver Fuel

MK-II -- Mark-II Fuel

P -- 1/2 Driver Fue1 - 1/2 Stainless Steel

$S$-- Safety Rod

SSCR -- Stainless Steel Control Rod

SSTH -- Stainless Steel Thimble

$X$-- Experimental Subassembly 


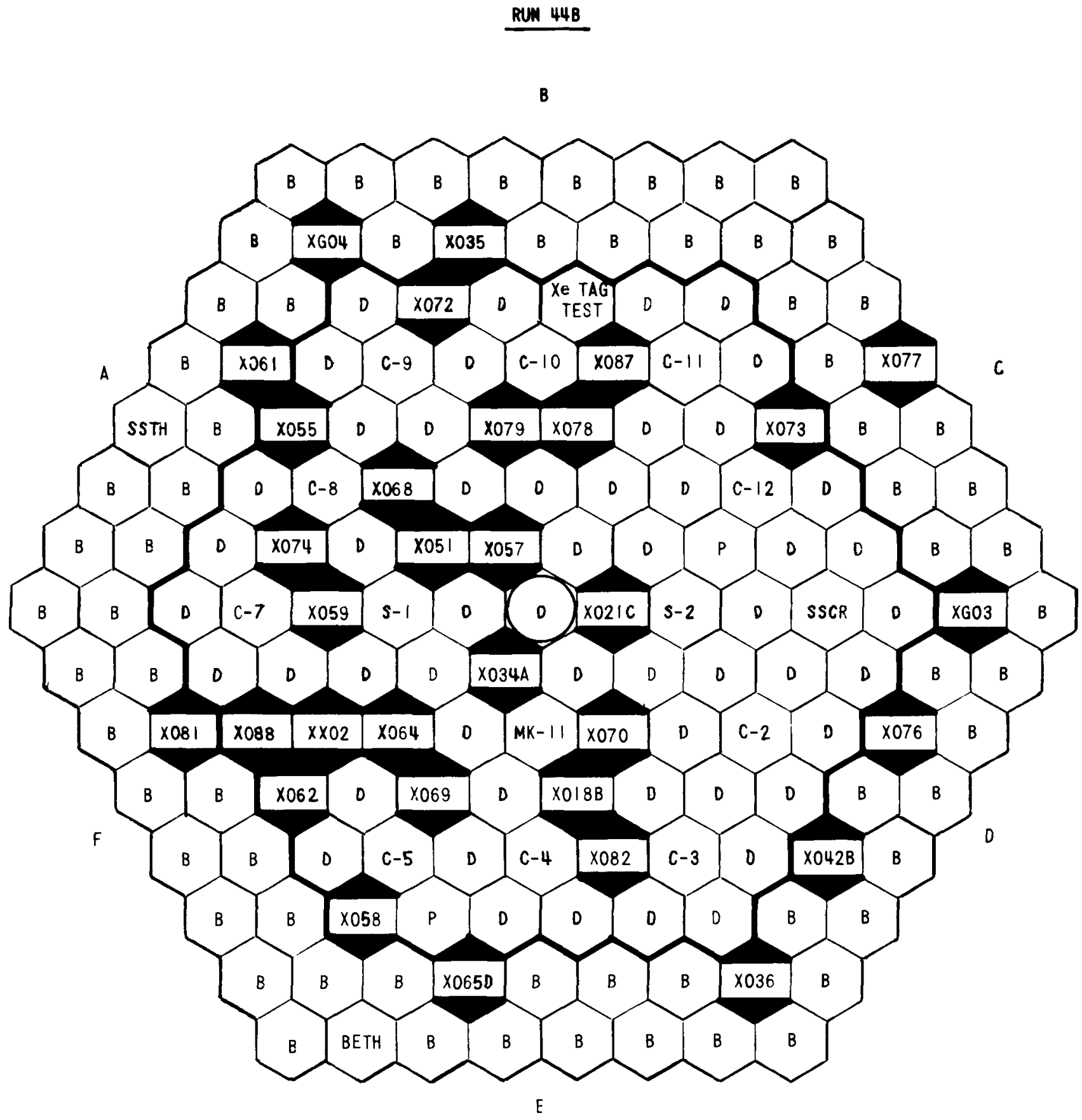


RUN 45A

(90-Subassemb1y Core Size)

Chronology

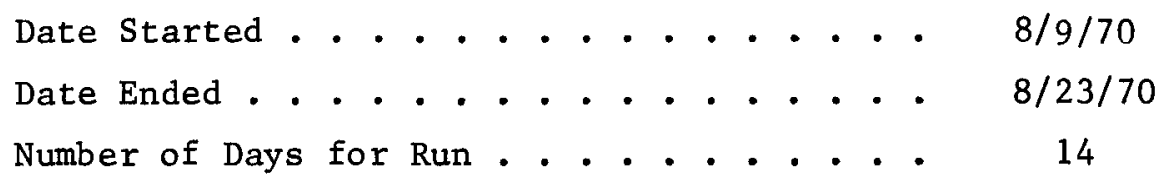

\section{Power Data}

Cumulative MWd(t) at Start of Run . . . 37,941

Cumulative MWd(t) at End of Run . . . 38,586

$\operatorname{MWd}(t)$ Generated During Run . . . . . . 645

Types of Subassemblies in Core

Blanket ............ . 23

Core ............... . 35

Control Rod ............ . 10

Safety $\operatorname{Rod} . . . . . . . . . . .22$

Experimentals (Rows 1-6)........ 17

Experimental Subassemblies in Row 7 . . . . . 6

Key to Types of Subassemblies Shown in Loading Diagram

B -- Depleted Uranium

BETH -- Beryllium Thimble

C -- Contro1 Rod

CF -- Controlled-flow Subassembly

D -- Driver Fue1

MK-II -- Mark-II Fuel

P - 1/2 Driver Fuel - 1/2 Stainless Steel

S - Safety Rod

SSCR -- Stainless Steel Control Rod

SSTH -- Stainless Steel Thimble

$\mathrm{X}$-- Experimental Subassembly 


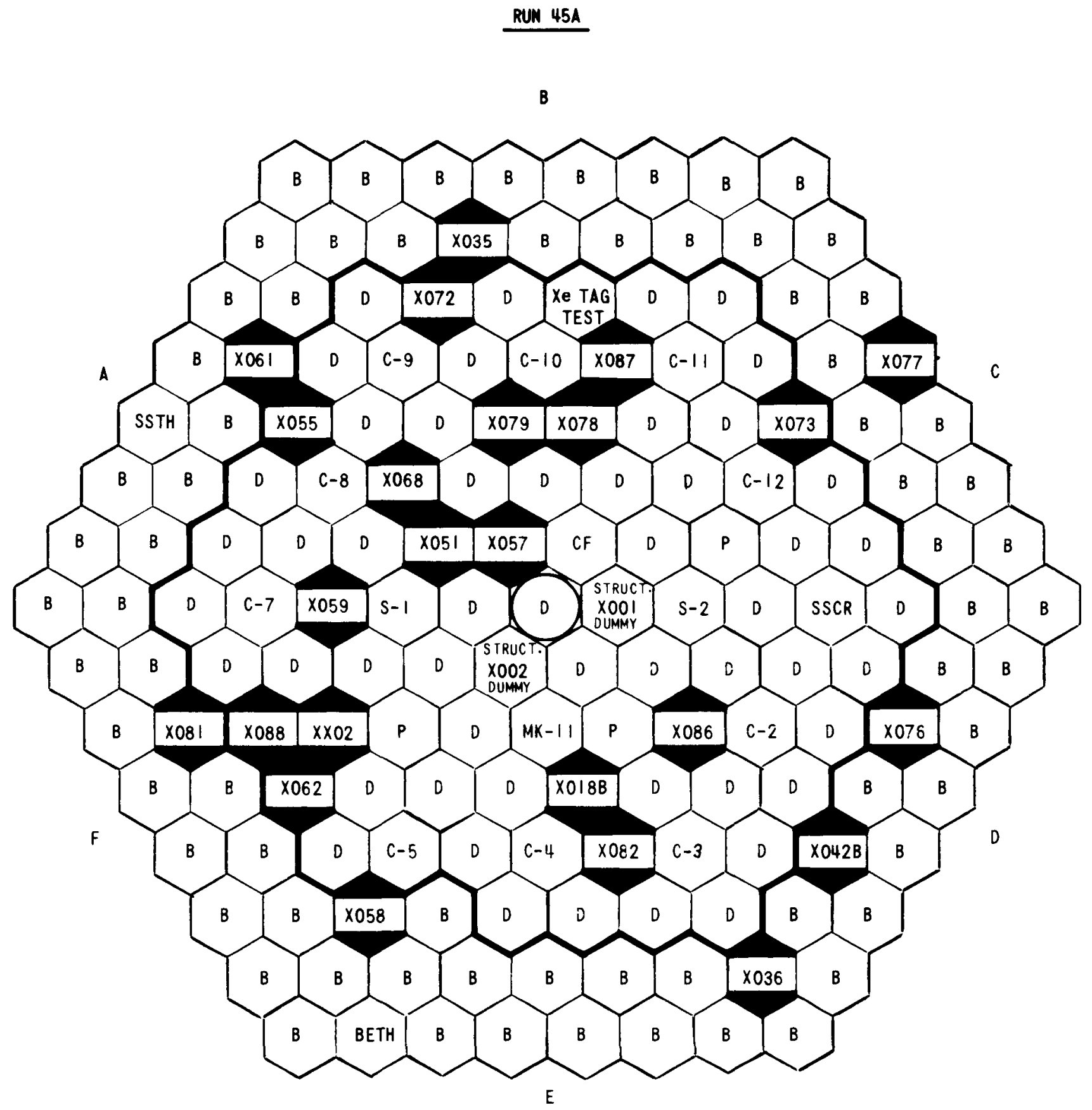


RUN 45B

(91-Subassembly Core Size)

Chronology

Date Started . . . . . . . . . 8/26/70

Date Ended ............... . $9 / 10 / 70$

Number of Days for Run . . . . . . . 15

$\underline{\text { Power Data }}$

Cumulative MWd(t) at Start of Run . . . . 38,586

Cumulative MWd $(t)$ at End of Run ..... 39,291

MWd $(t)$ Generated During Run . . . . . 705

Types of Subassemblies in Core

Blanket . . . . . . . . . . . 24

Core .............. . . 35

Control Rod .............. 10

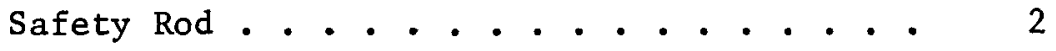

Experimentals (Rows 1-6) . . . . . . 17

Experimental Subassemblies in Row 7 . . . . . 5

Key to Types of Subassemblies Shown in Loading Diagram

B -- Depleted Uranium

BETH -- Beryllium Thimble

C -- Control Rod

D -- Driver Fuel

P - 1/2 Driver Fuel - 1/2 Stainless Steel

$S$-- Safety Rod

SSCR -- Stainless Steel Control Rod

SSTH -- Stainless Steel Thimble

$\mathrm{X}$-- Experimental Subassembly 


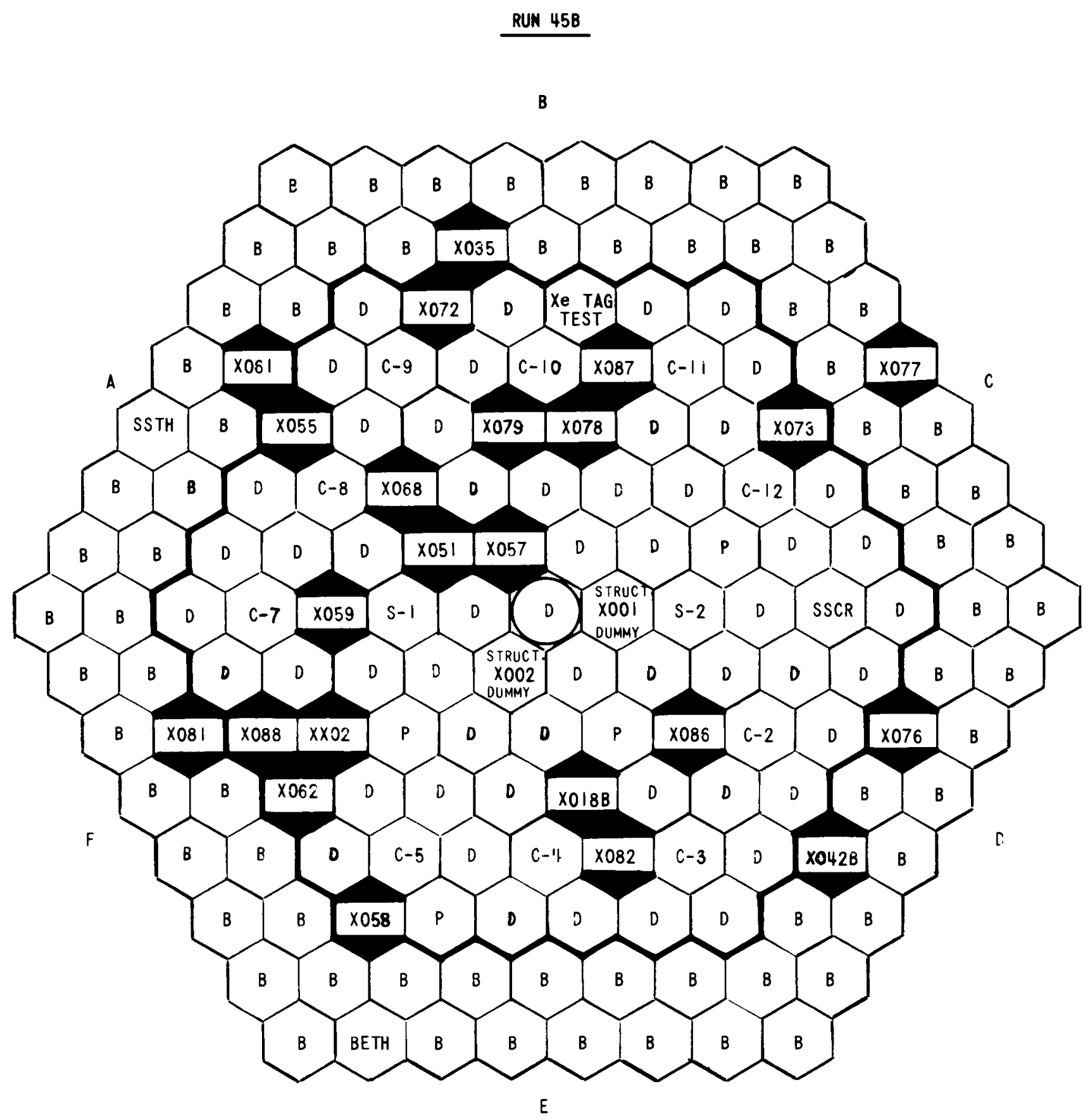


$\underline{\text { RUN 46A }}$

(88-Subassembly Core Size)

\section{Chronology}

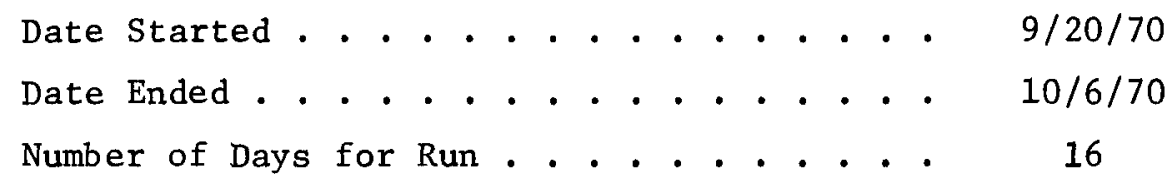

\section{Power Data}

Cumulative MWd(t) at Start of Run . . . . 39,291

Cumulative MWd( $t$ ) at End of Run .. . . . 40,042

$\operatorname{MWd}(t)$ Generated During Run . . . . . 751

\section{Types of Subassemblies in Core}

Blanket ............ . 26

Core . . . . . . . . . . . 39

Control Rod ............. 10

Safety $\operatorname{Rod} . . . . . . . . . .22$

Experimentals (Rows 1-6) . . . . . . . 7

Experimental Subassemblies in Row 7 . . . . . 11

Key to Types of Subassemblies Shown in Loading Diagram

B -- Depleted Uranium

BETH -- Beryllium Thimble

C -- Control Rod

D -- Driver Fue1

MK-II -- Mark-II Fuel

P -- 1/2 Driver Fuel - 1/2 Stainless Steel

R -- Stainless Steel Reflector

$S$-- Safety Rod

SSCR -- Stainless Steel Control Rod

SSTH -- Stainless Steel Thimble

$X$-- Experimental Subassembly 


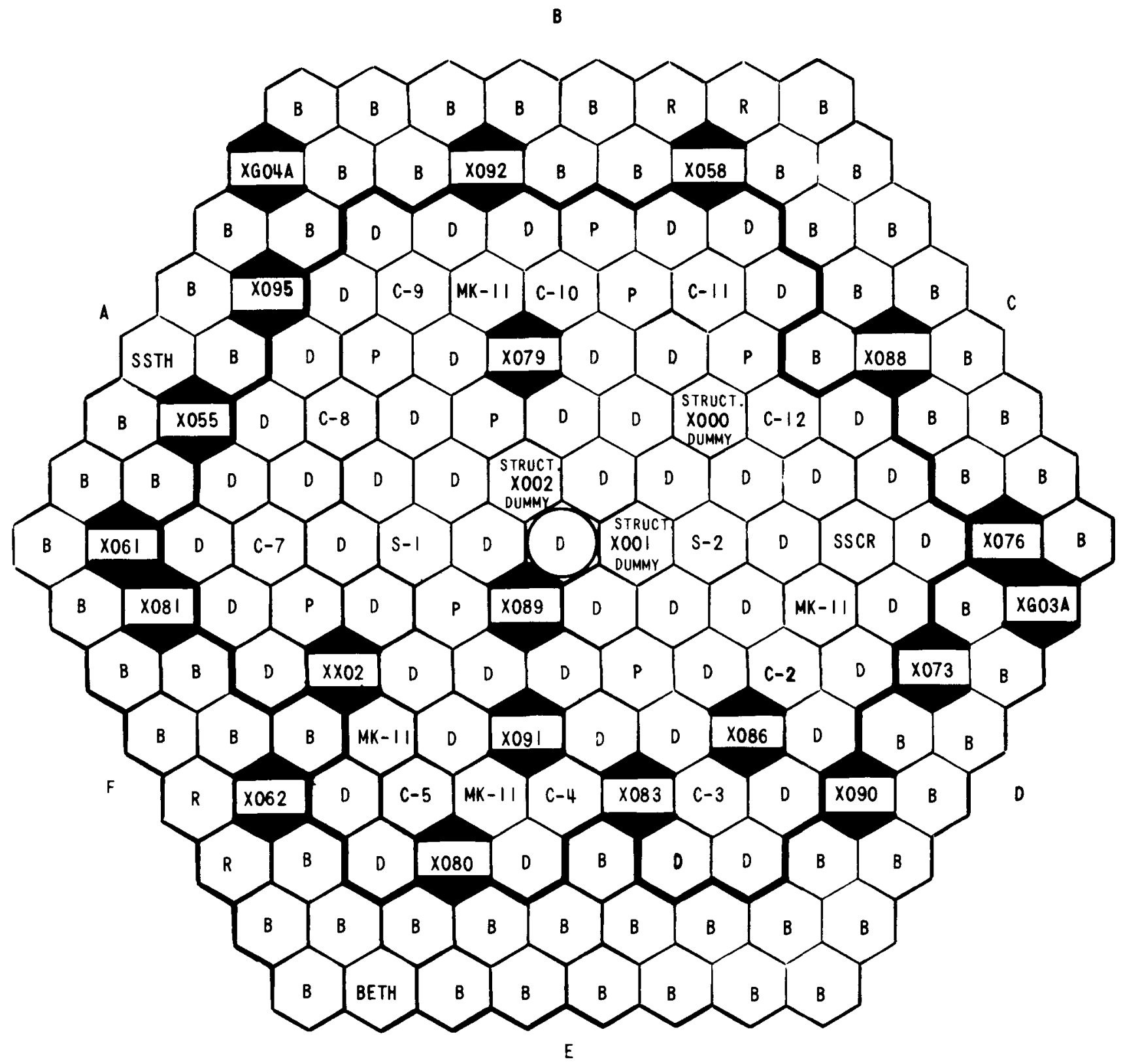


RUN 46B

(88-Subassembly Core Size)

Chronology

Date Started ............ 10/8/70

Date Ended . . . . . . . . . . 10/22/70

Number of Days for Run . . . . . . . 14

\section{Power Data}

Cumulative MWd(t) at Start of Run . . . . 40,042

Cumulative MWd(t) at End of Run ... . . 40,841

MWd $(t)$ Generated During Run . . . . . 799

Types of Subassemblies in Core

Blanket . . . . . . . . . 26

Core . . . . . . . . . . . 39

Control Rod ............. . 11

Safety $\operatorname{Rod} . . . . . . . . . .22$

Experimentals (Rows 1-6) . . . . . . . . 9

Experimental Subassemblies in Row 7 . . . . . 10

Key to Types of Subassemblies Shown in Loading Diagram

B -- Depleted Uranium

BETH - Beryllium Thimble

C -- Control Rod

D -- Driver Fuel

Mk-II -- Mark-II Fue1

P -- 1/2 Driver Fuel - 1/2 Stainless Steel

R -- Stainless Steel Reflector

S -- Safety Rod

SSTH -- Stainless Steel Thimble

$\mathrm{X}$-- Experimental Subassembly 


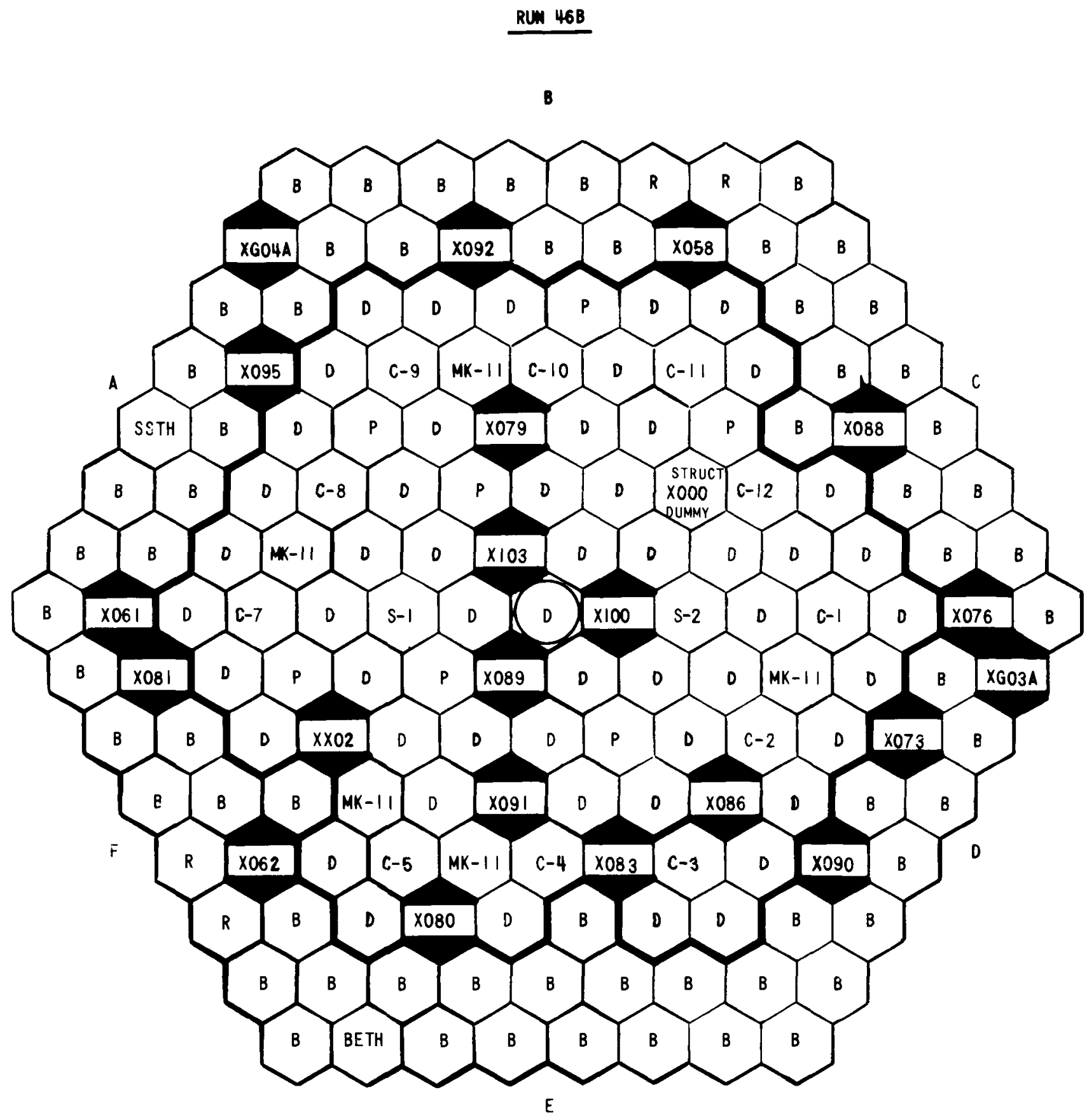




\section{RUN 47A}

(89-Subassemb1y Core Size)

Chronology

Date Started .............. 10/28/70

Date Ended .............. 11/15/70

Number of Days for Run . . . . . . . . 18

Power Data

Cumulative MWd(t) at Start of Run . . . . 40,841

Cumulative MWd(t) at End of Run . . . . 4 41,816

MWd $(t)$ Generated During Run . . . . . . 975

Types of Subassemblies in Core

Blanket . . . . . . . . . . . . 23

Core . . . . . . . . . . . 38

Control Rod . . . . . . . . . . . 10

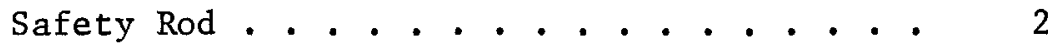

Experimentals (Rows 1-6) . . . . . . . . 13

Experimental Subassemblies in Row 7 . . . . . 11

Key to Types of Subassemblies Shown in Loading Diagram

B -- Depleted Uranium

BETH -- Beryllium Thimble

C -- Control Rod

D -- Driver Fuel

MK-II -- Mark-II Fue1

P -- 1/2 Driver Fuel - 1/2 Stainless Stee1

R -- Stainless Steel Reflector

S -- Safety Rod

SSCR -- Stainless Steel Control Rod

SSTH -- Stainless Steel Thimble

$\mathrm{X}$-- Experimental Subassemb1y 


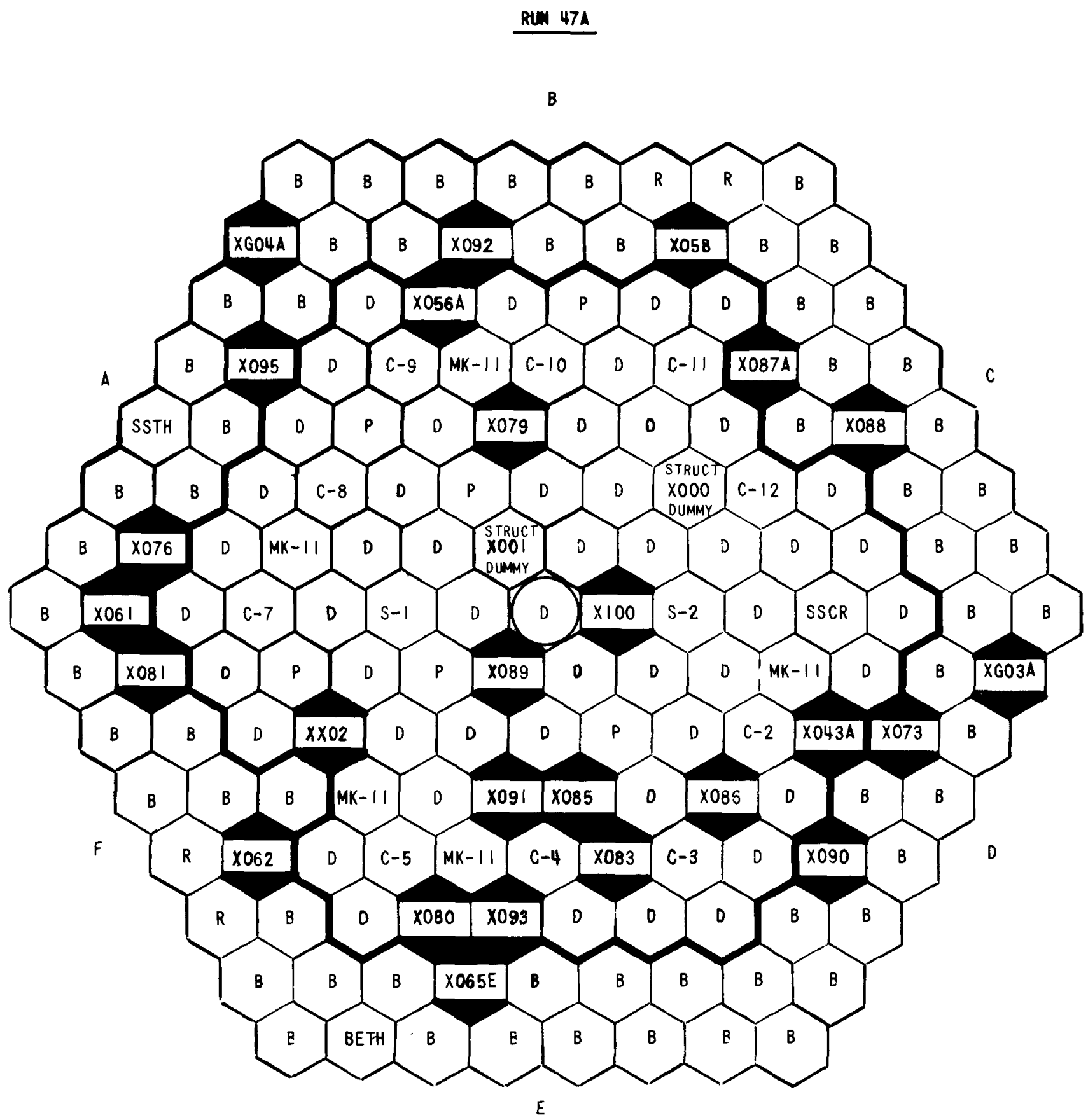


$\underline{\text { RUN 47B }}$

(91-Subassembly Core Size)

Chronology

Date Started . . . . . . . . . . 3/2/71

Date Ended . . . . . . . . . . 3 3/13/71

Number of Days for Run . . . . . . . . 11

\section{Power Data}

Cumulative MWd(t) at Start of Run . . . . 41,816

Cumulative MWd(t) at End of Run . . . . . 42,321

MWd( $t$ ) Generated During Run ....... 505

Types of Subassemblies in Core

Blanket . . . . . . . . . . 22

Core ................. 37

Control Rod ............. 10

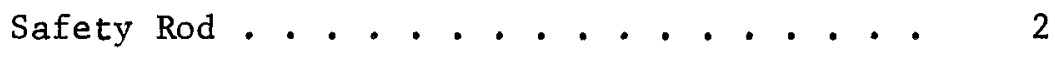

Experimentals (Rows 1-6)........ . 19

Experimental Subassemblies in Row 7 . . . . . 15

Key to Types of Subassemblies Shown in Loading Diagram

B -- Depleted Uranium

BETH -- Beryllium Thimble

C -- Control Rod

D -- Driver Fuel

MK-II -- Mark-II Fuel

P -- 1/2 Driver Fue1 - 1/2 Stainless Stee1

R -- Stainless Steel Reflector

$S$-- Safety Rod

SSCR -- Stainless Steel Control Rod

SSTH -- Stainless Steel Thimble

$\mathrm{X}$-- Experimental Subassembly 


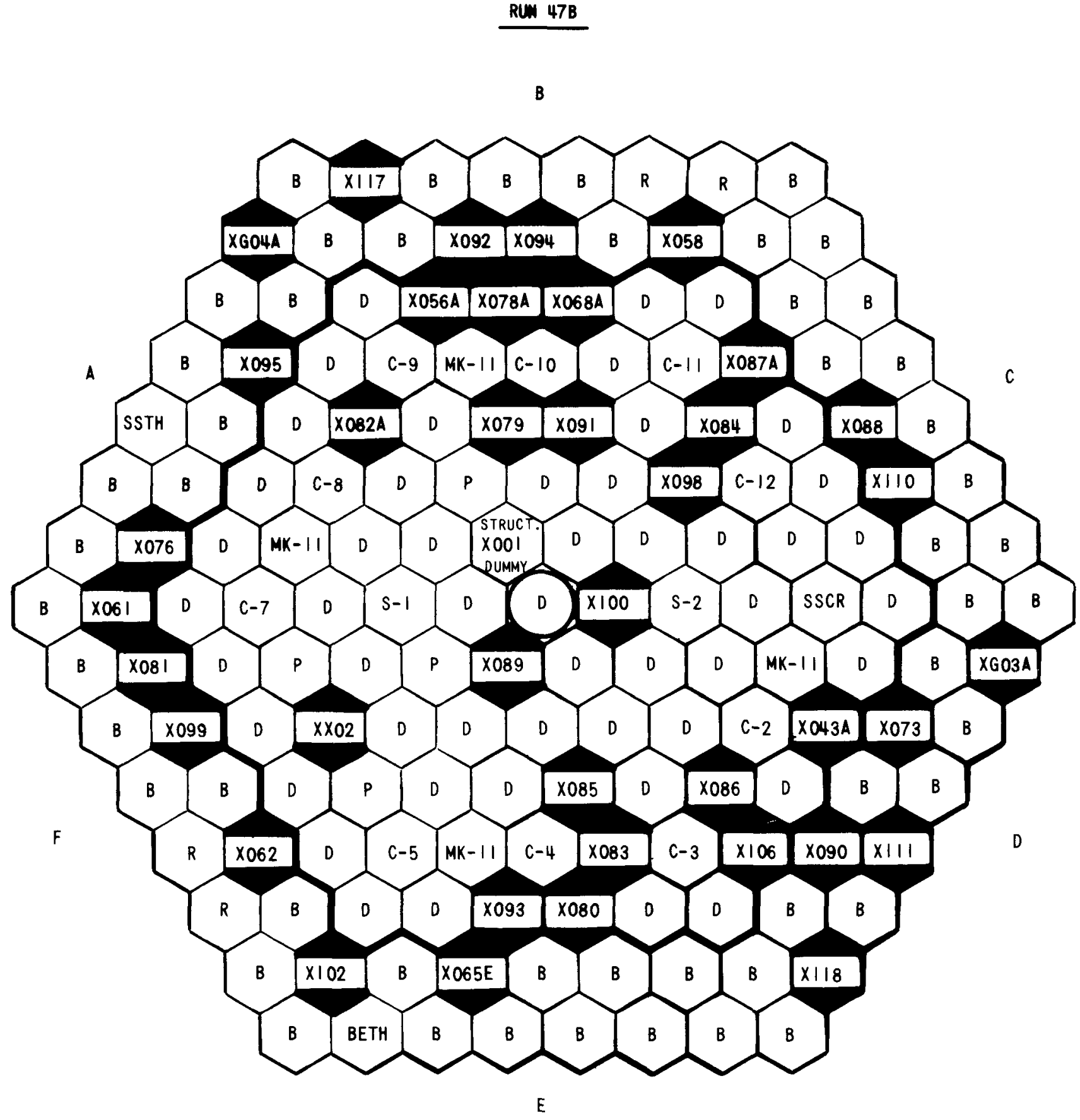


$\underline{\text { RUN } 48 \mathrm{~A}}$

(88-Subassembly Core Size)

Chronology

Date Started . . . . . . . . . 3/31/71

Date Ended . . . . . . . . . . 4/2/71

Number of Days for Run ......... . 3

\section{Power Data}

Cumulative MWd(t) at Start of Run . . . . 42,321

Cumulative $\operatorname{MWd}(t)$ at End of Run ..... 42,340

MWd (t) Generated During Run ........ 19

Types of Subassemblies in Core

Blanket . . . . . . . . . . 20

Core ............... . 38

Control Rod .............. . 9

Safety Rod . . . . . . . . . . . 2

Experimentals (Rows 1-6) . . . . . . . 16

Experimental Subassemblies in Row 7 . . . . . 13

Key to Types of Subassemblies Shown in Loading Diagram

B -- Depleted Uranium

BETH -- Beryllium Thimble

C -- Control Rod

D -- Driver Fuel

MK-II -- Mark-II Fue1

OSC -- Oscillator Rod

P - 1/2 Driver Fue1 - 1/2 Stainless Stee1

R -- Stainless Steel Reflector

S -- Safety Rod

SSTH -- Stainless Steel Thimble

$\mathrm{X}$-- Experimental Subassembly 


\section{RUN 48A}

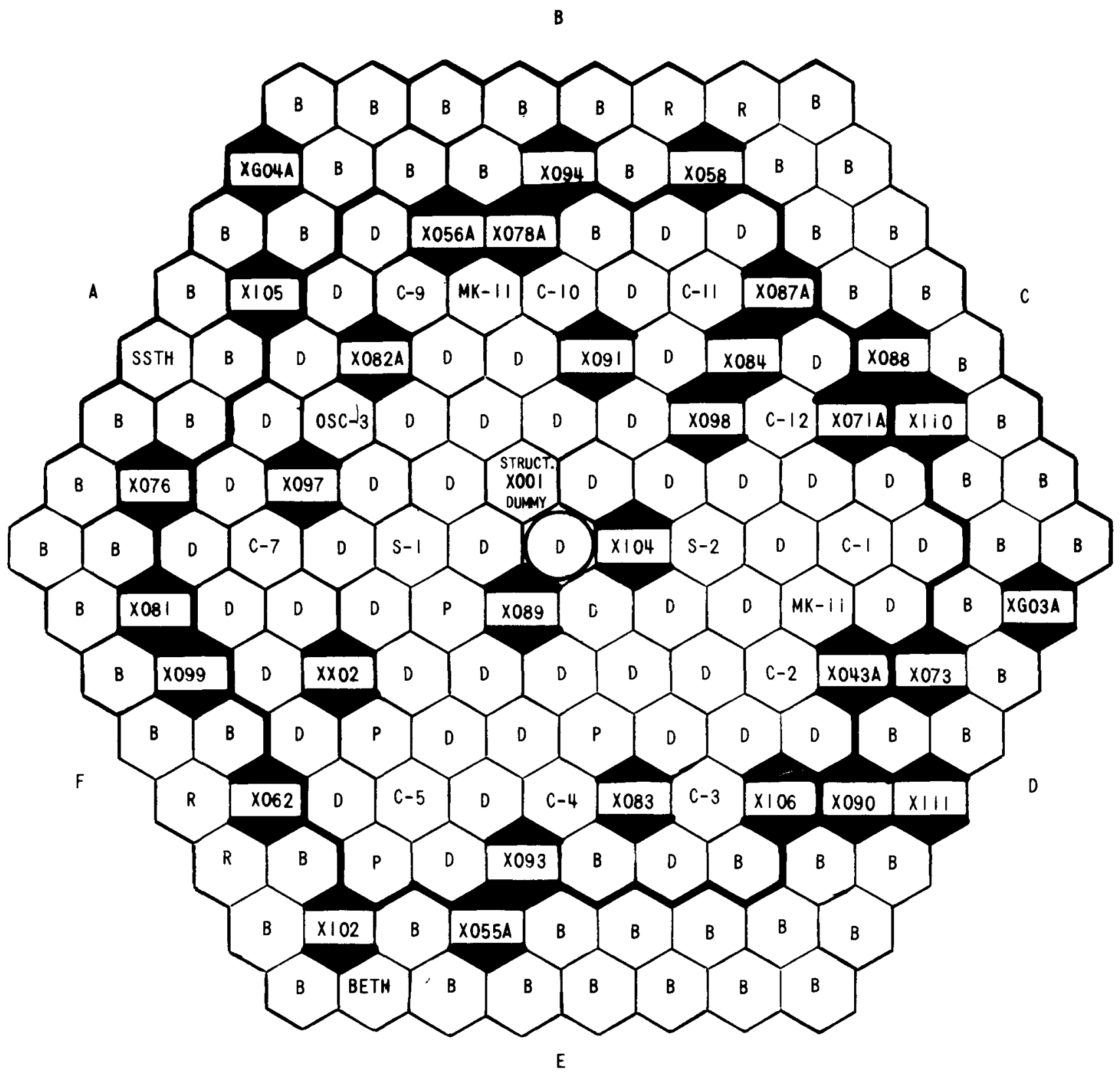


RUN 48B

(88-Subassembly Core Size)

Chronology

Date Started . . . . . . . . . 4/3/71

Date Ended . . . . . . . . . . 4/4/71

Number of Days for Run .......... . 1

Power Data

Cumulative MWd(t) at Start of Run . . . . 42,340

Cumulative MWd( $t$ ) at End of Run . . . . 42,400

MWd $(t)$ Generated During Run . . . . . . 60

\section{Types of Subassemblies in Core}

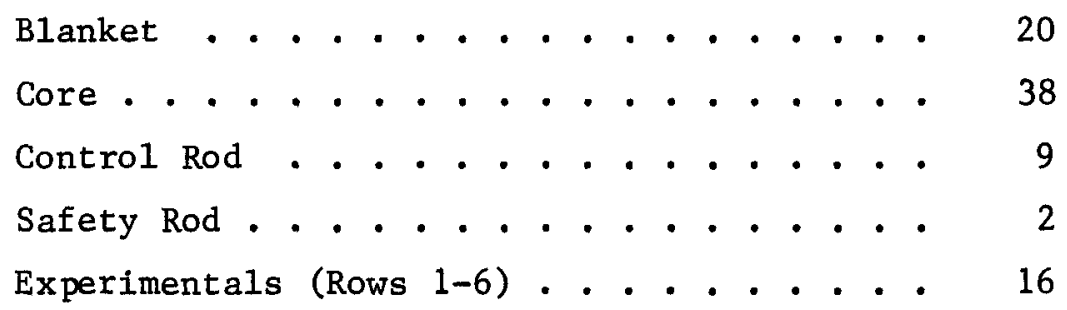

Experimental Subassemblies in Row 7...... 13

Key to Types of Subassemblies Shown in Loading Diagram

B -- Depleted Uranium

BETH -- Beryllium Thimble

C -- Control Rod

D -- Driver Fue1

MK-II -- Mark-II Fuel

OSC -- Oscillator Rod

P -- 1/2 Driver Fuel - 1/2 Stainless Steel

R -- Stainless Steel Reflector

S -- Safety Rod

SSCR -- Stainless Steel Control Rod

SSTH -- Stainless Steel Thimble

$X$-- Experimental Subassemb1y 


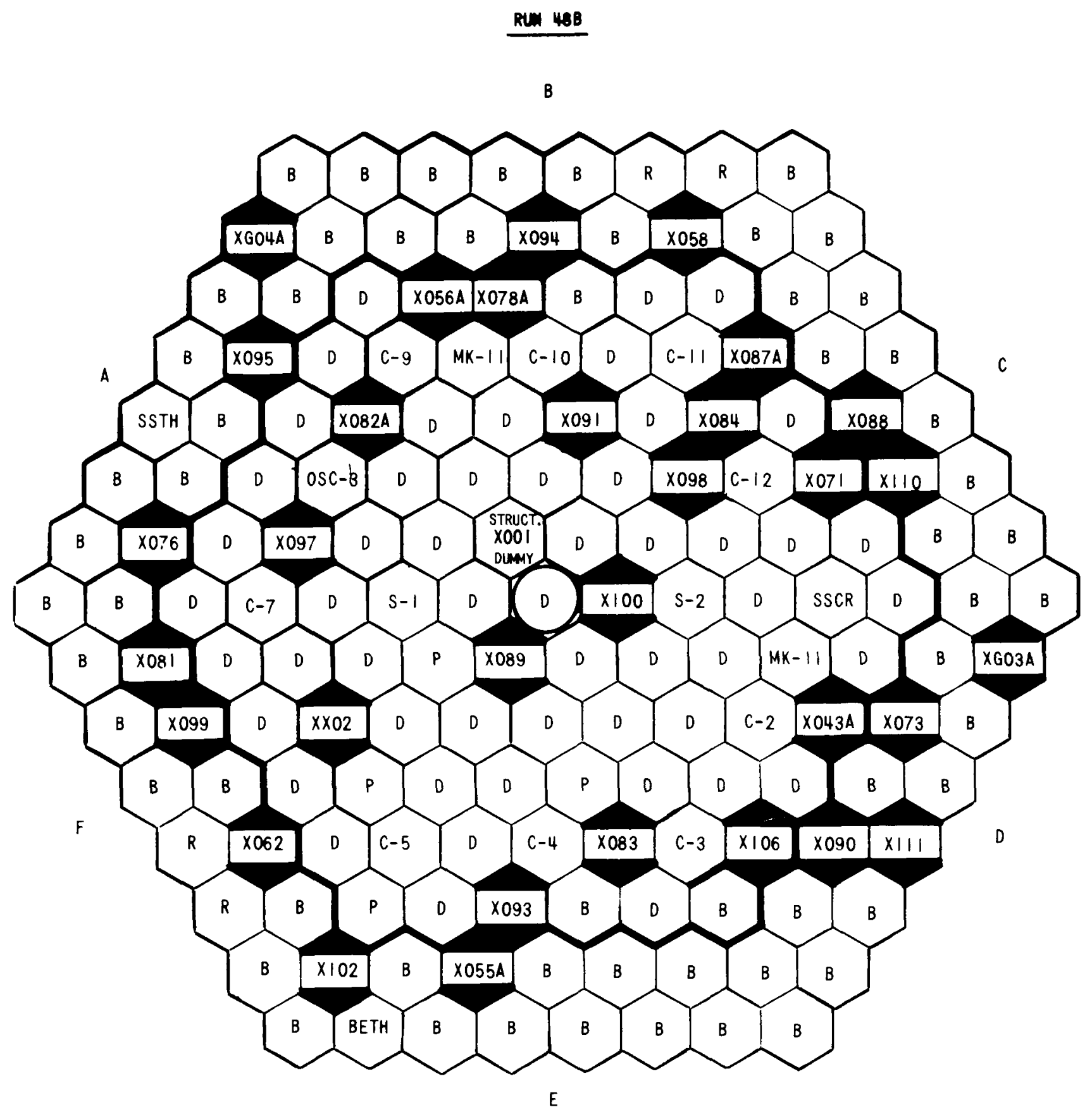


RUN 48C

(91-Subassembly Core Size)

\section{Chronology}

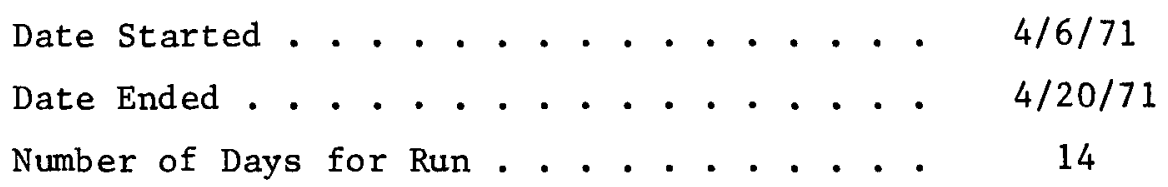

\section{Power Data}

Cumulative MWd(t) at Start of Run . . . . 42,400

Cumulative MWd( $t$ ) at End of Run . . . . 43,126

MWd $(t)$ Generated During Run . . . . . 726

Types of Subassemblies in Core

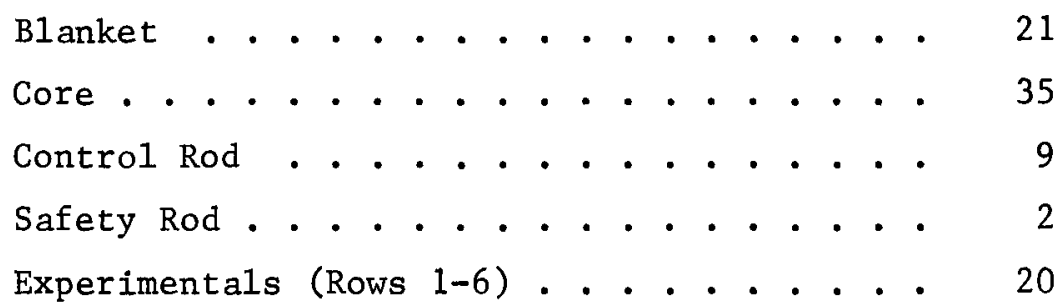

Experimental Subassemblies in Row 7 . . . . . 9

Key to Types of Subassemblies Shown in Loading Diagram

B -- Depleted Uranium

BETH -- Beryllium Thimble

C -- Control Rod

D -- Driver Fuel

MK-II -- Mark-II Fuel

OSC -- Oscillator Rod

P -- 1/2 Driver Fuel - 1/2 Stainless Steel

R -- Stainless Steel Reflector

S -- Safety Rod

SSCR -- Stainless Steel Control Rod

SSTH -- Stainless Steel Thimble

X -- Experimental Subassembly 


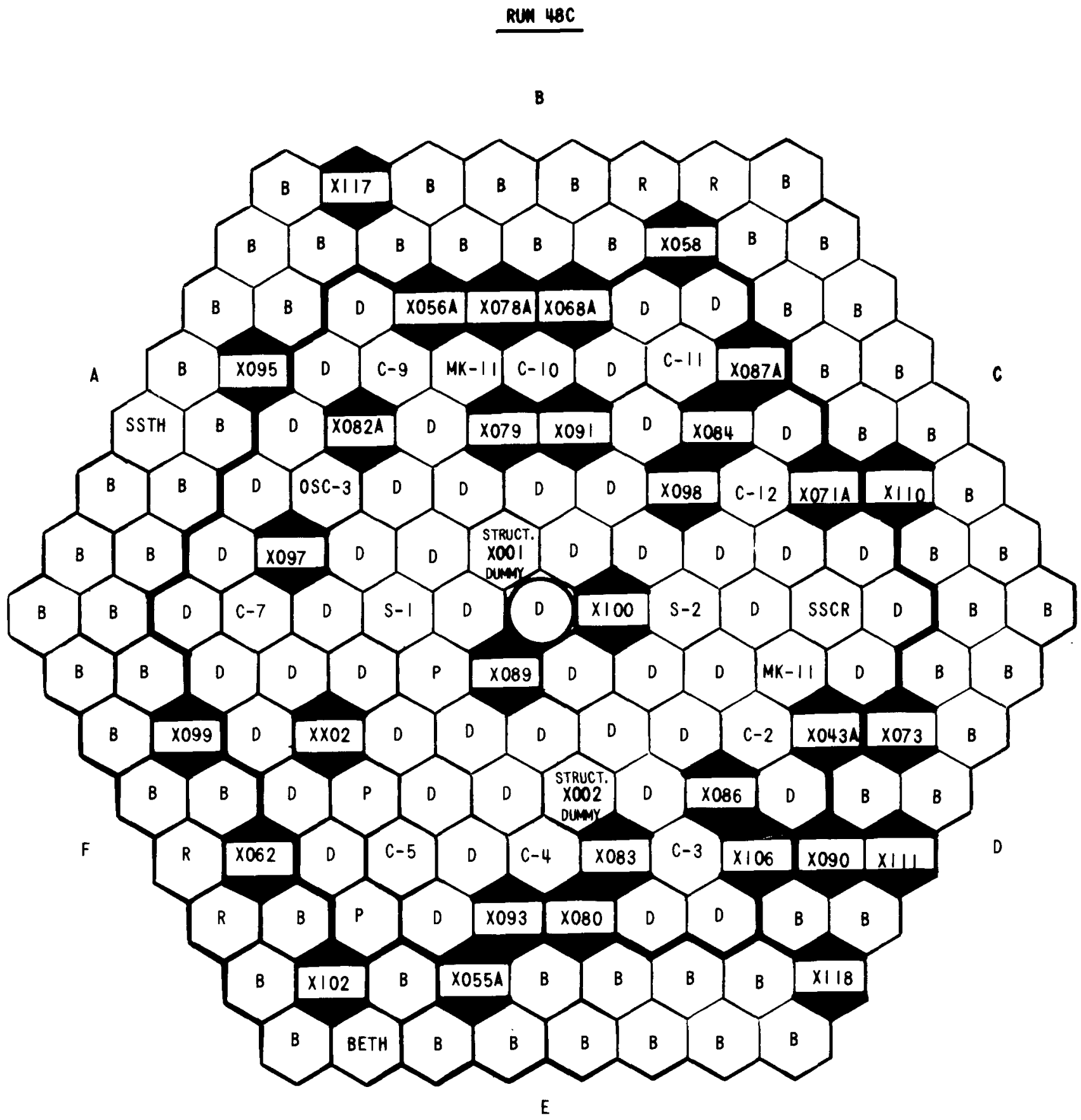


$\underline{\mathrm{RUN}}$ 48D

(91-Subassembly Core Size)

Chronology

Date Started .............. 4/22/71

Date Ended .............. 5/4/71

Number of Days for Run . . . . . . . 12

Power Data

Cumulative MWd(t) at Start of Run . . . 43,126

Cumulative MWd $(t)$ at End of Run . . . . 43,776

MWd $(t)$ Generated During Run . . . . . . 650

Types of Subassemblies in Core

Blanket ............. 16

Core . . . . . . . . . . . 36

Control Rod .............. 9

Safety $\operatorname{Rod} . . . . . . . . . . .22$

Experimentals (Rows 1-6) . . . . . . 25

Experimental Subassemblies in Row 7 . . . . . 9

Key to Types of Subassemblies Shown in Loading Diagram

B -- Depleted Uranium

BETH -- Beryllium Thimble

C -- Control Rod

D -- Driver Fue1

MK-II -- Mark-II Fue1

OSC -- Oscillator Rod

P -- 1/2 Driver Fuel - 1/2 Stainless Stee1

R -- Stainless Steel Reflector

$S$-- Safety Rod

SSCR -- Stainless Steel Contro1 Rod

SSTH -- Stainless Steel Thimble

X -- Experimental Subassembly 


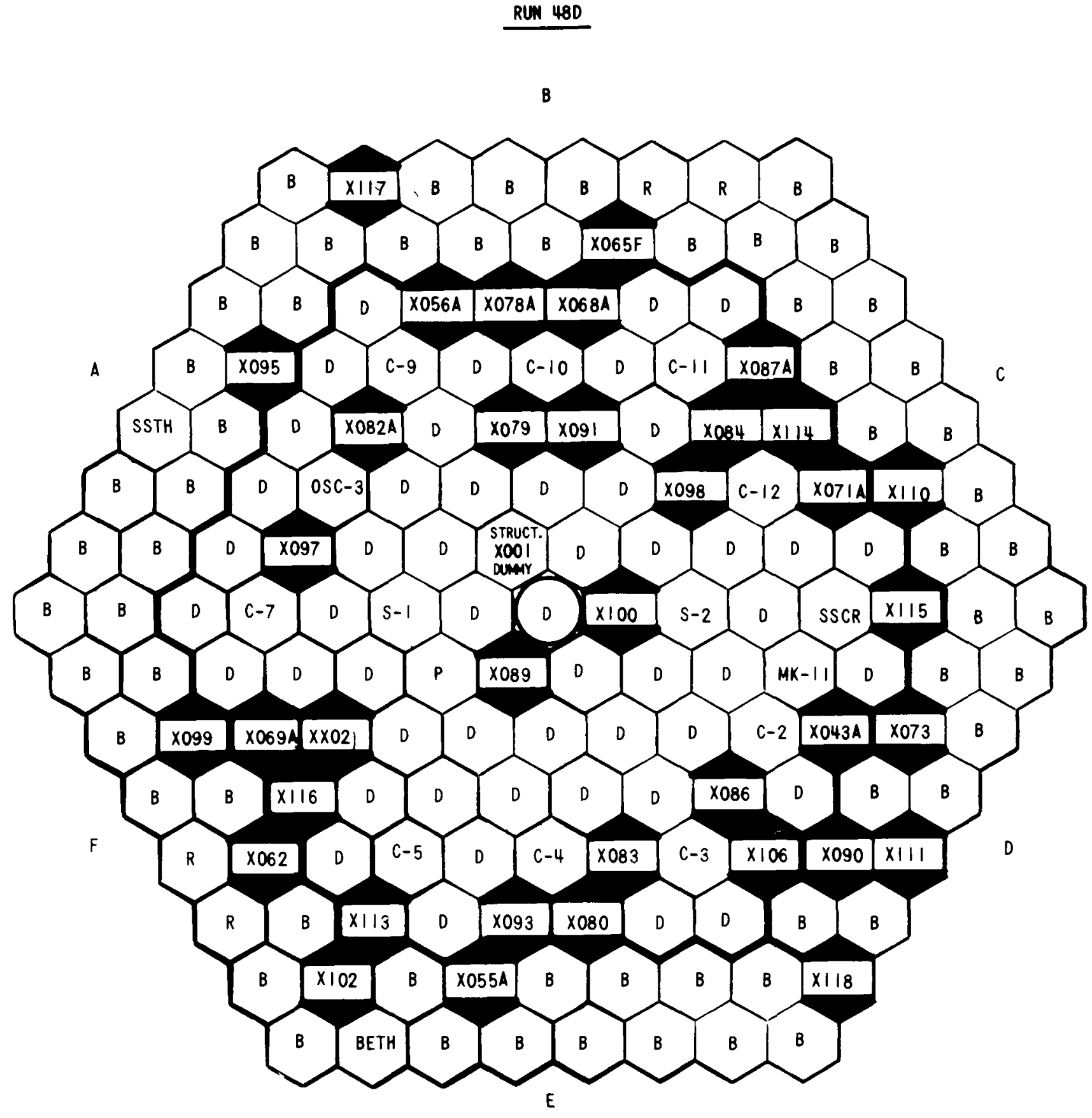


$\underline{\text { RUN } 48 \mathrm{E}}$

(91-Subassembly Core Size)

Chronology

Date Started .............. 5/6/71

Date Ended .............. 5/7/71

Number of Days for Run . . . . . . . $12.5 \mathrm{hr}$

\section{Power Data}

Cumulative MWd(t) at Start of Run . . . 4 43,776

Cumulative MWd $(t)$ at End of Run ..... 43,788

MWd $(t)$ Generated During Run . . . . . 12

Types of Subassemblies in Core

Blanket . . . . . . . . . . 16

Core . . . . . . . . . . 36

Control Rod . . . . . . . . . . 9

Safety Rod .............. 2

Experimentals (Rows 1-6) . . . . . . 26

Experimental Subassemblies in Row 7 . . . . . 9

Key to Types of Subassemblies Shown in Loading Diagram

B -- Depleted Uranium

BETH -- Beryllium Thimble

C -- Control Rod

D -- Driver Fuel

MK-II -- Mark-II Fuel

OSC -- Oscillator Rod

P -- 1/2 Driver Fue1 - 1/2 Stainless Steel

R -- Stainless Steel Reflector

$S$-- Safety Rod

SSCR -- Stainless Steel Control Rod

SSTH -- Stainless Steel Thimble

X -- Experimental Subassembly 


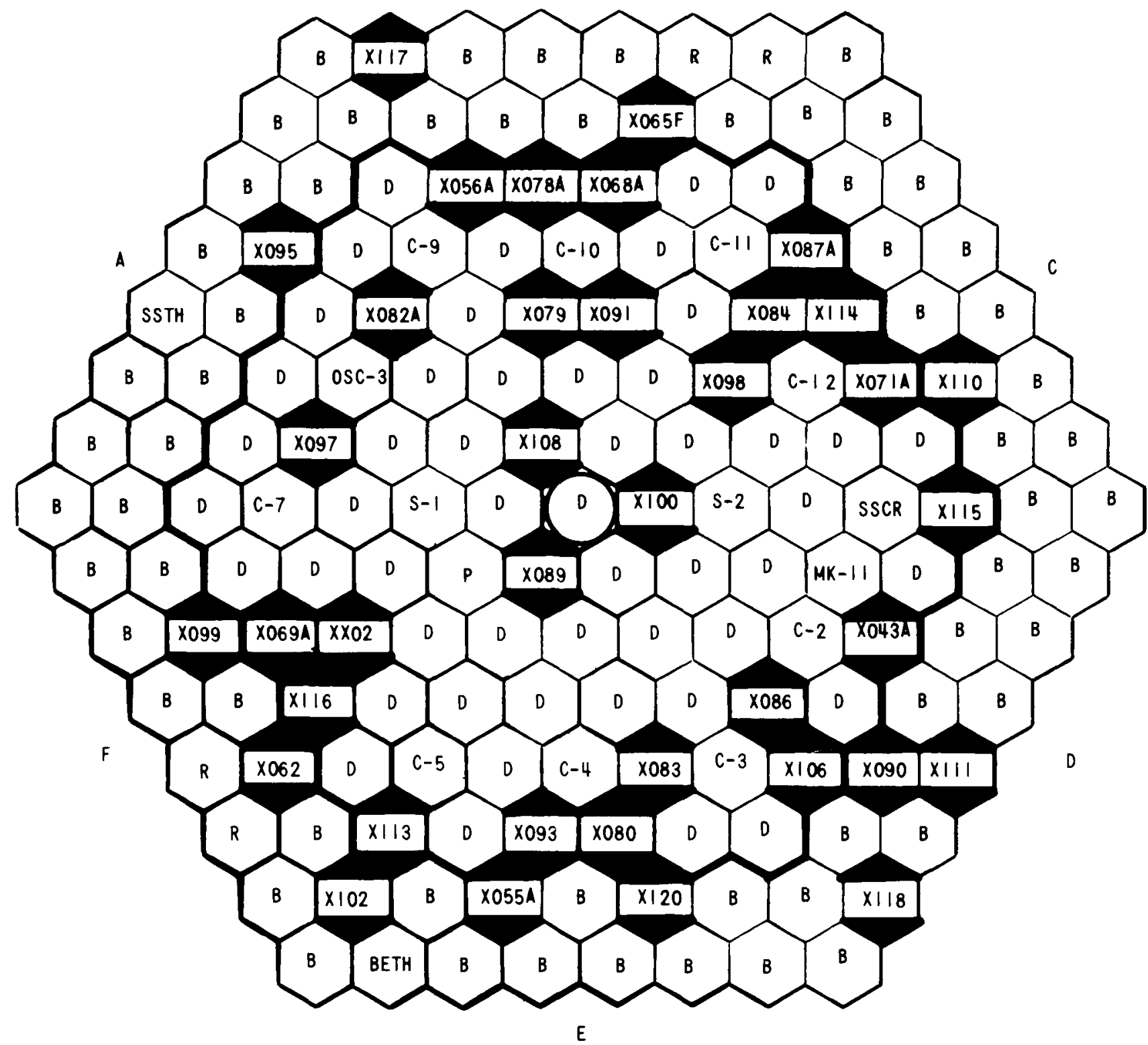


RUN 49A

$$
\text { (91-Subassemb1y Core Size) }
$$

\section{Chronology}

Date Started . . . . . . . . . . 5 5/18/71

Date Ended . . . . . . . . . . . 5/19/71

Number of Days for Run .......... 1

\section{Power Data}

Cumulative MWd(t) at Start of Run . . . . 43,788

Cumulative MWd(t) at End of Run . . . . 43,852

MWd $(t)$ Generated During Run . . . . . . 64

\section{Types of Subassemblies in Core}

Blanket . . . . . . . . . . 16

Core . . . . . . . . . . . . 35

Control Rod .............. . 9

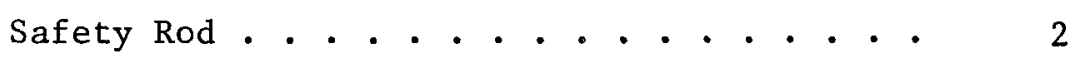

Experimentals (Rows 1-6) . . . . . . 27

Experimental Subassemblies in Row 7 . . . . . . 14

Key to Types of Subassemblies Shown in Loading Diagram

B -- Depleted Uranium

BETH -- Beryllium Thimble

C -- Control Rod

D -- Driver Fuel

MK-II -- Mark-II Fue1

OSC -- Oscillator Rod

R -- Stainless Steel Reflector

$S$-- Safety Rod

SSCR -- Stainless Steel Thimble

$X$-- Experimental Subassembly 


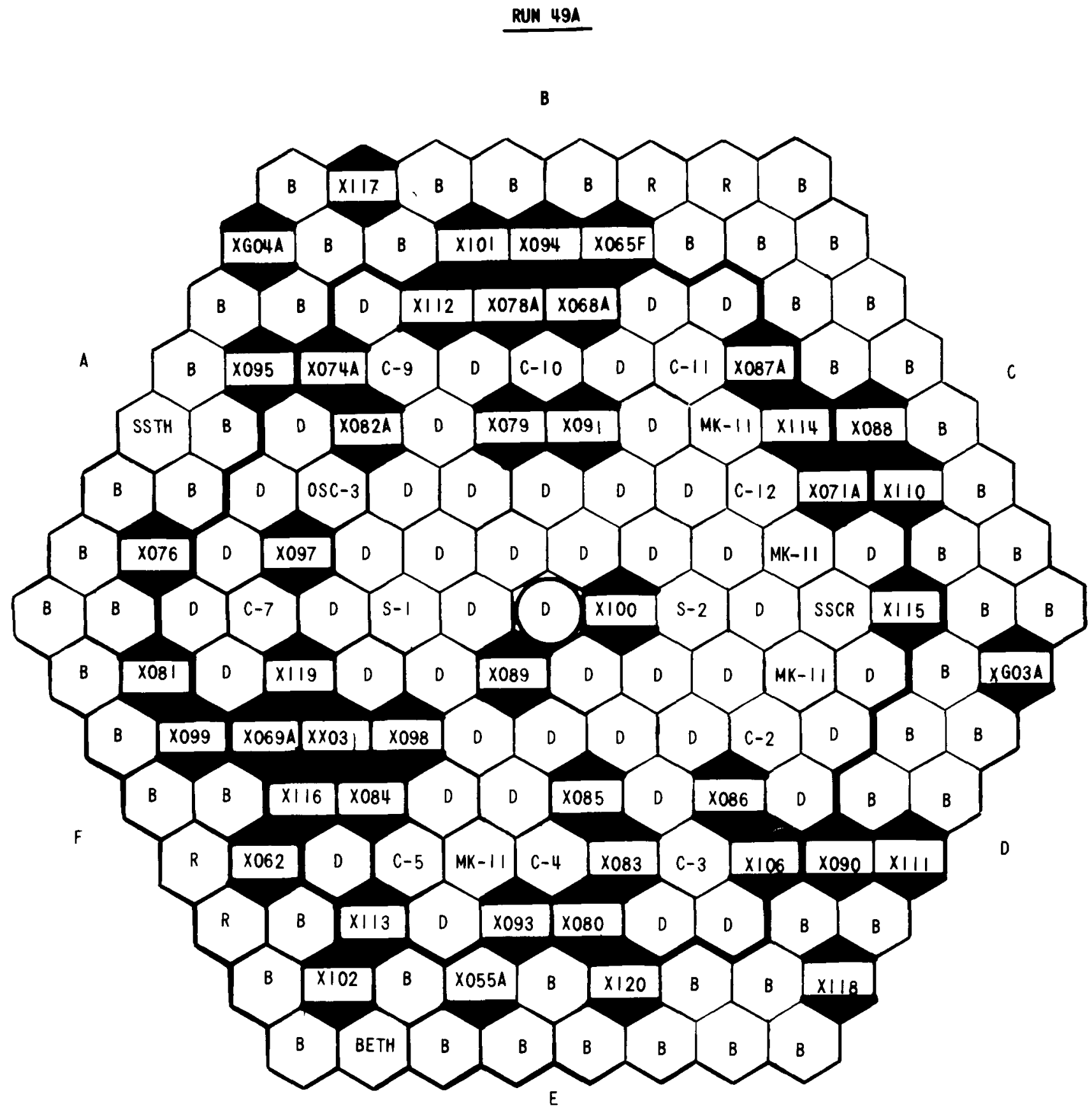


RUN 49B

(91-Subassembly Core Size)

Chronology

Date Started . . . . . . . . . . 5/20/71

Date Ended . . . . . . . . . . . 5 5/31/71

Number of Days for Run . . . . . . . . 11

Power Data

Cumulative MWd(t) at Start of Run . . . 43,852

Cumulative MWd( $t$ ) at End of Run ..... 44,442

MWd $(t)$ Generated During Run ....... 590

Types of Subassemb1ies in Core

Blanket ............. . . 16

Core . . . . . . . . . . . . 35

Control Rod . . . . . . . . . . . 9

Safety Rod . . . . . . . . . . . . 2

Experimentals (Rows 1-6) . . . . . . . 27

Experimental Subassemblies in Row 7 . . . . . . 14

Key to Types of Subassemblies Shown in Loading Diagram

B -- Depleted Uranium

BETH -- Beryllium Thimble

C -- Control Rod

D -- Driver Fuel

MK-II -- Mark-II Fuel

OSC -- Oscillator Rod

R -- Stainless Steel Reflector

S -- Safety Rod

SSCR -- Stainless Steel Control Rod

SSTH -- Stainless Steel Thimble

X -- Experimental Subassembly 


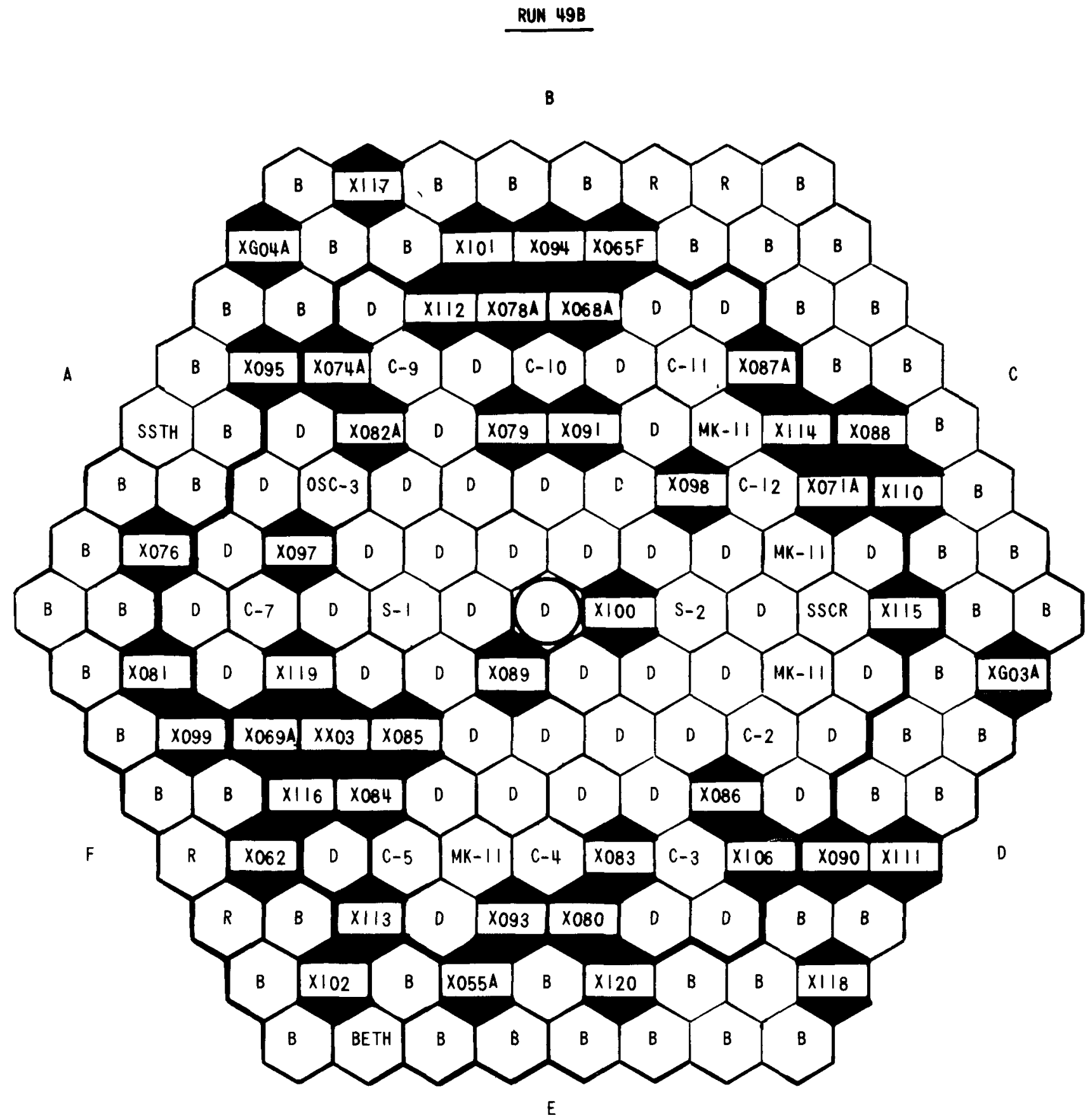


RUN 49C

(91-Subassemb1y Core Size)

\section{Chronology}

Date Started .............. 6/3/71

Date Ended .............. . . 6/8/71

Number of Days for Run ........... 5

\section{Power Data}

Cumulative MWd(t) at Start of Run . . . . 44,442

Cumulative MWd(t) at End of Run . . . . 44,716

MWd $(t)$ Generated During Run . . . . . . 274

\section{Types of Subassemblies in Core}

Blanket ............ . . 16

Core ............... . . 36

Contro1 Rod .............. . . 9

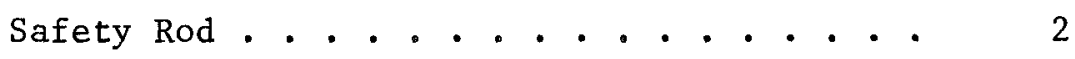

Experimentals (Rows 1-6) . . . . . . 26

Experimental Subassemblies in Row 7 . . . . . 14

Key to Types of Subassemblies Shown in Loading Diagram

B -- Depleted Uranium

BETH -- Beryllium Thimble

C -- Control Rod

D -- Driver Fuel

MK-II -- Mark-II Fuel

OSC -- Oscillator Rod

P -- 1/2 Driver Fue1 - 1/2 Stainless Stee1

R -- Stainless Stee1 Reflector

$S$-- Safety Rod

SSCR -- Stainless Steel Control Rod

SSTH -- Stainless Steel Thimble

$\mathrm{X}$-- Experimental Subassembly 


\section{RUN $49 C$}

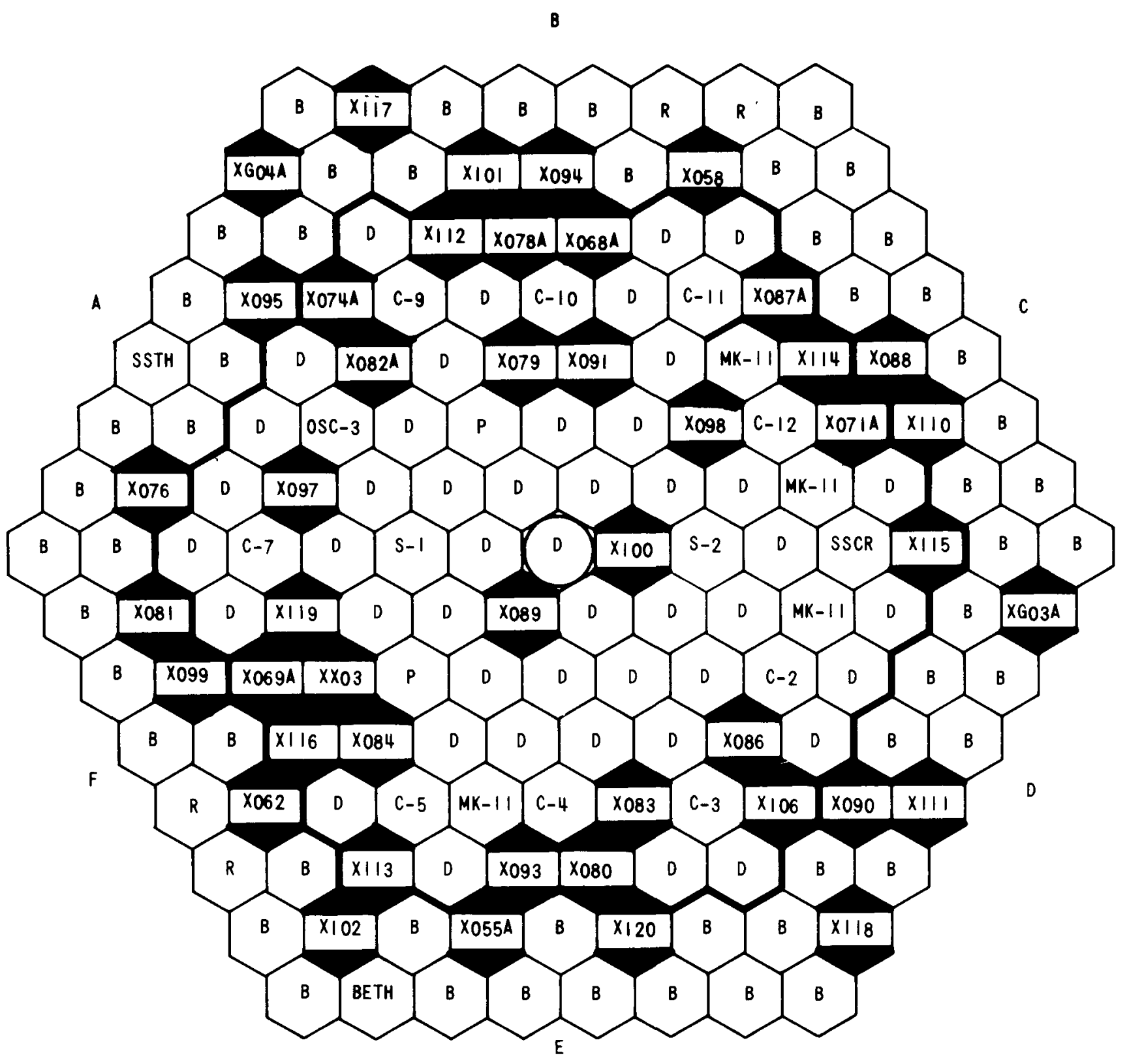


RUN 49D

(90-Subassemb1y Core Size)

\section{Chronology}

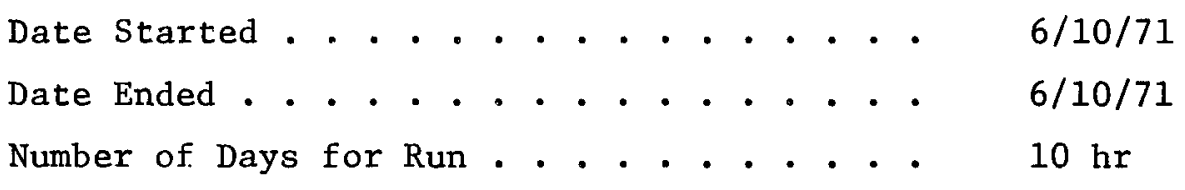

Power Data

Cumulative MWd(t) at Start of Run . . . . 44,716

Cumulative MWd( $t$ ) at End of Run . . . . . 44,721

MWd $(t)$ Generated During Run . . . . . . 5

\section{Types of Subassemblies in Core}

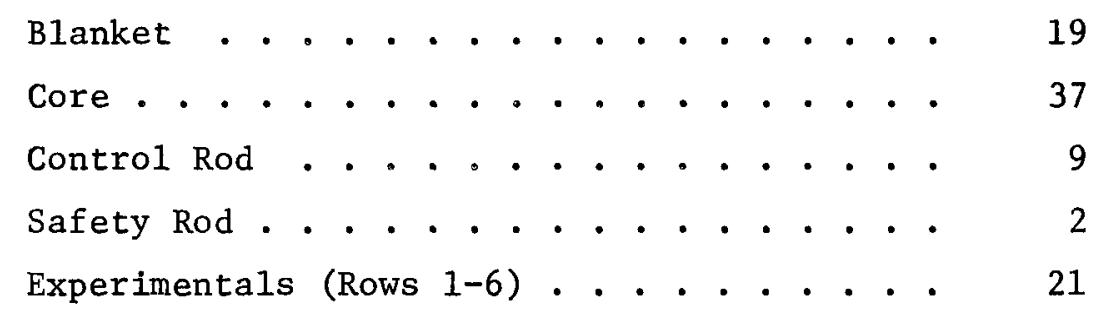

Experimental Subassemblies in Row 7 . . . . . 13

Key to Types of Subassemblies Shown in Loading Diagram

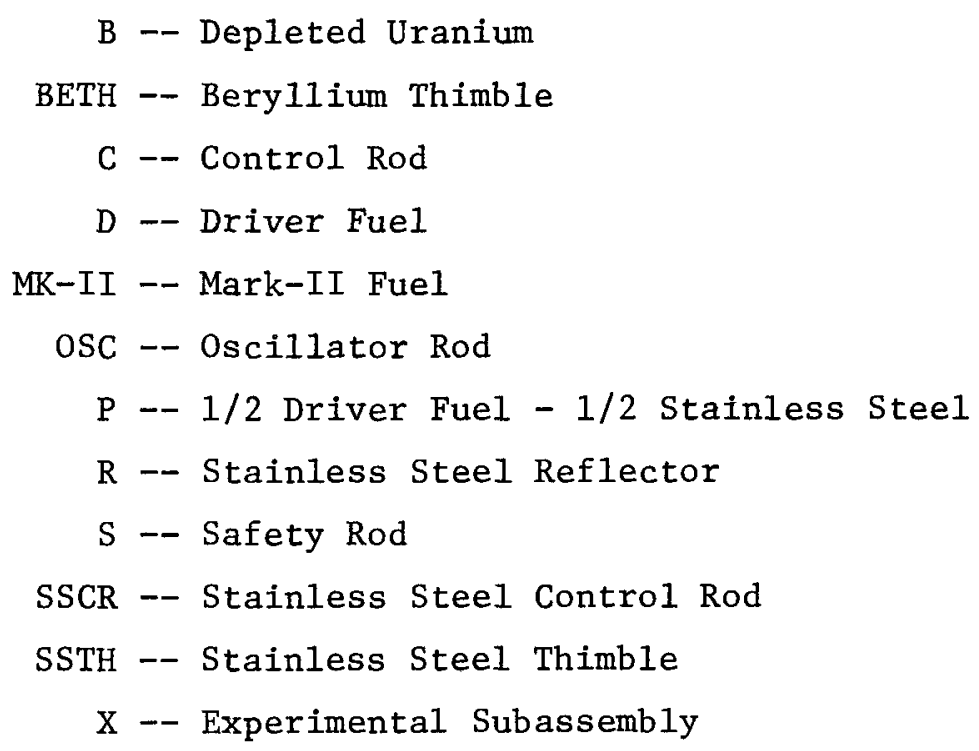




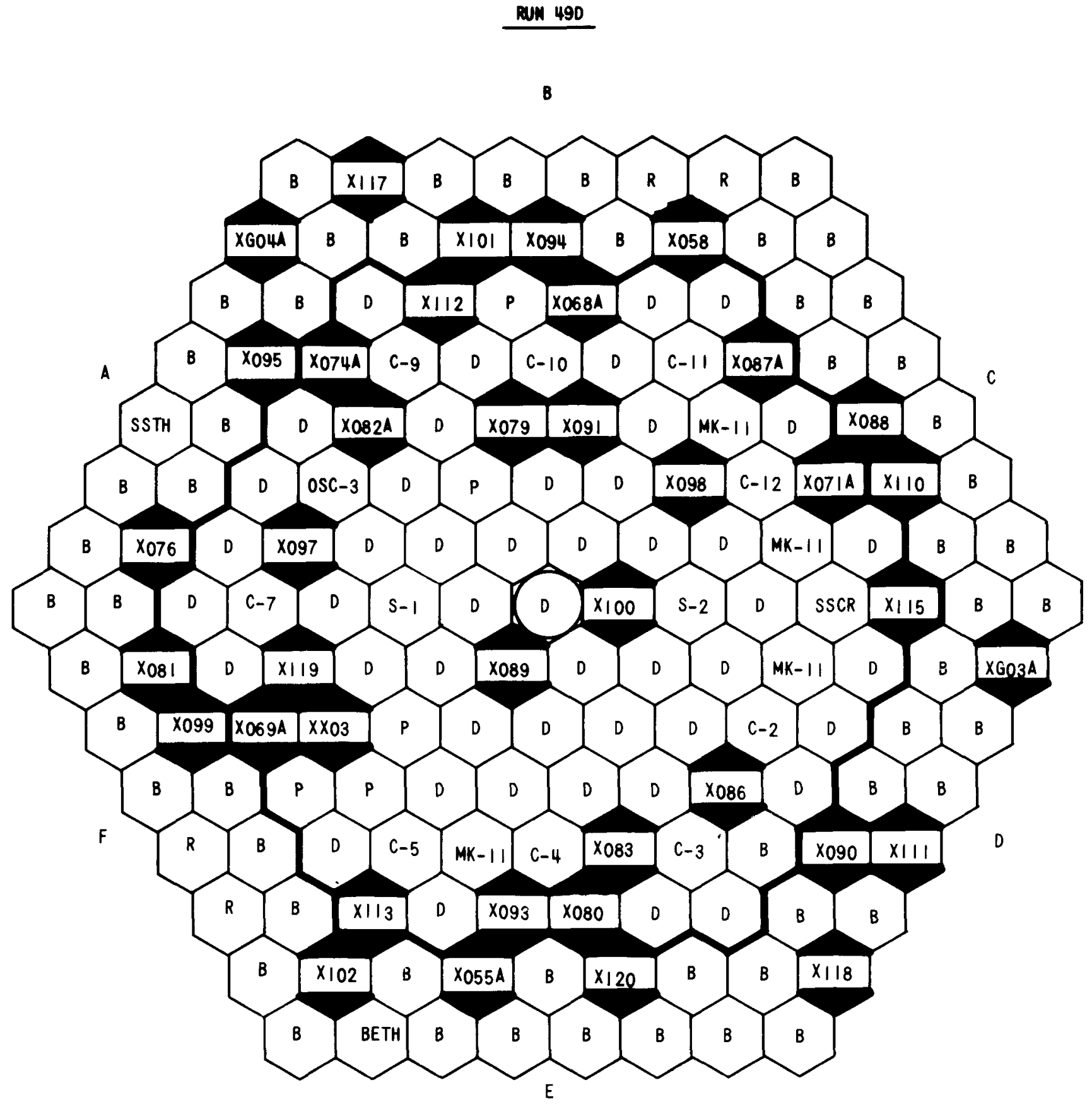


RUN 49E

(89-Subassembly Core Size)

Chronology

Date Started . . . . . . . . . . . . . . .
Date Ended . . . . . . . . . . . . . . . .
Number of Days for Run . . . . . . . . . . .

Power Data

Cumulative MWd(t) at Start of Run . . . . 44,721

Cumulative MWd( $t$ ) at End of Run . . . . 4 44,734

MWd $(t)$ Generated During Run . . . . . . 13

Types of Subassemblies in Core

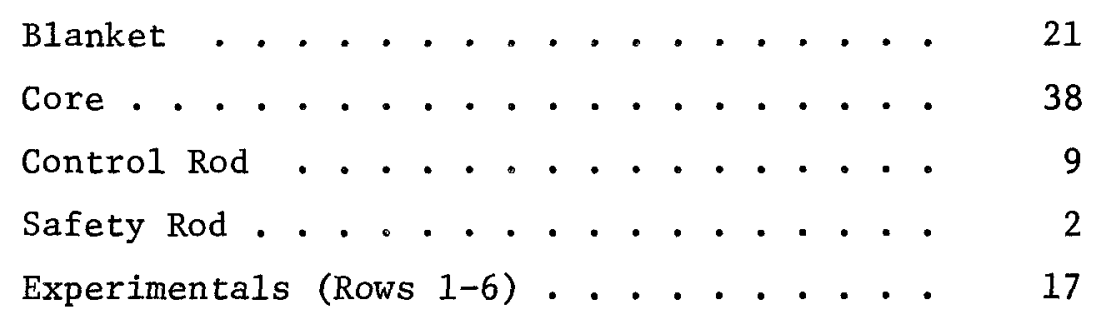

Experimental Subassemblies in Row 7 . . . . . 12

Key to Types of Subassemblies Shown in Loading Diagram

B -- Depleted Uranium

BETH -- Beryllium Thimble

C -- Control Rod

D -- Driver Fue1

MK-II -- Mark-II Fuel

OSC -- Oscillator Rod

P -- 1/2 Driver Fuel - 1/2 Stainless Steel

R -- Stainless Steel Reflector

S -- Safety Rod

SSCR -- Stainless Steel Control Rod

SSTH -- Stainless Steel Thimble

X -- Experimental Subassembly 


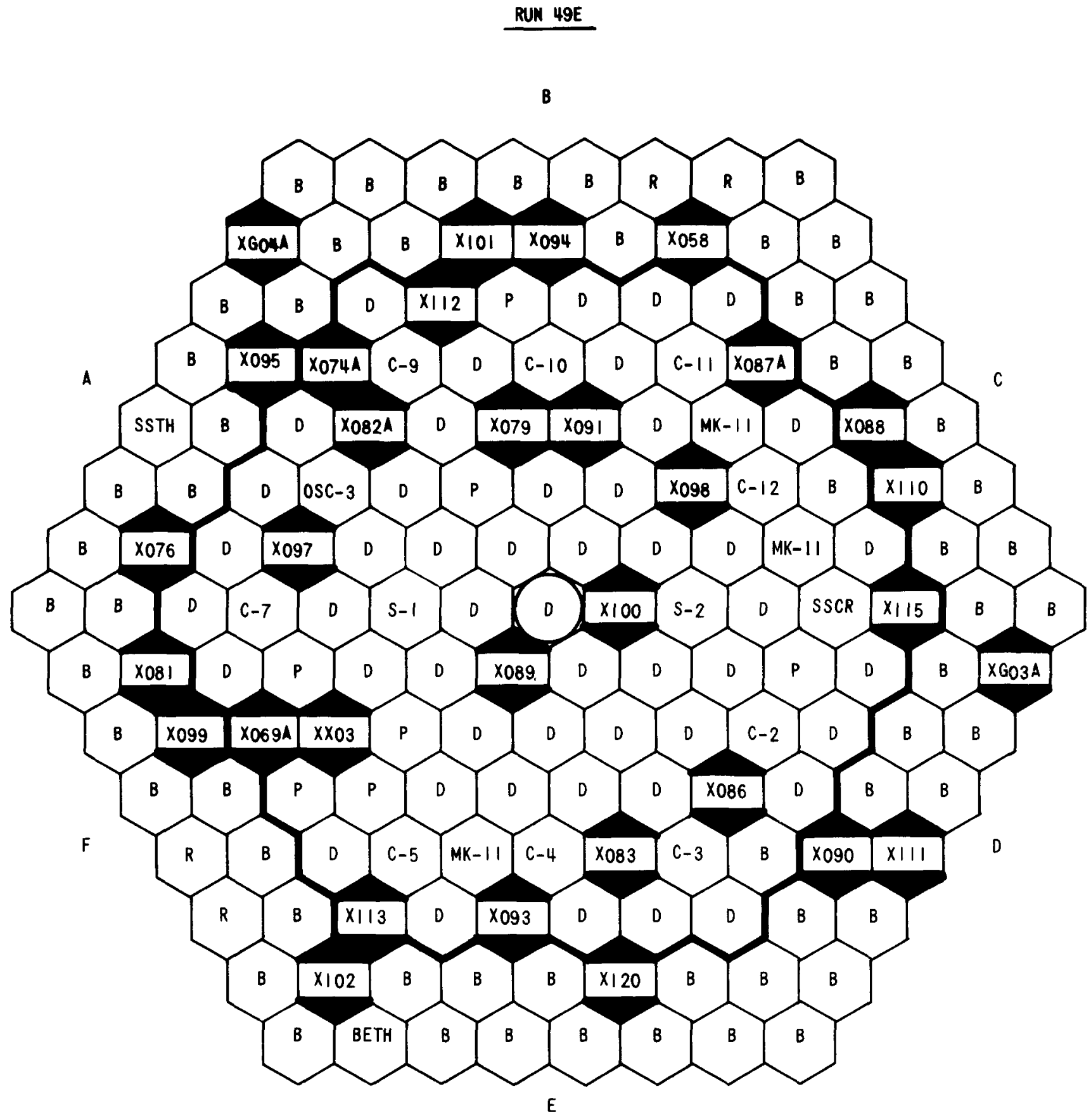


RUN 49F

(89-Subassemb1y Core Size)

Chronology

Date Started . . . . . . . . . . 6/20/71

Date Ended . . . . . . . . . . 6/30/71

Number of Days for Run . . . . . . . 10

Power Data

Cumulative MWd(t) at Start of Run . . . . 44,734

Cumulative MWd( $t$ ) at End of Run . . . . . 45,299

MWd $(t)$ Generated During Run . . . . . . 565

Types of Subassemblies in Core

Blanket . . . . . . . . . 20

Core ................. . . 4 42

Control Rod . . . . . . . . . . 9

Safety Rod ............... 2

Experimenta1s (Rows 1-6) . . . . . . . 14

Experimenta1 Subassemblies in Row 7 . . . . . 11

Key to Types of Subassemblies Shown in Loading Diagram

B -- Depleted Uranium

BETH -- Beryllium Thimble

C -- Contro1 Rod

D -- Driver Fuel

OSC -- Oscillator Rod

P -- 1/2 Driver Fuel - 1/2 Stainless Stee1

R -- Stainless Steel Reflector

S -- Safety Rod

SSCR -- Stainless Steel Control Rod

SSTH -- Stainless Steel Thimble

$\mathrm{X}$-- Experimental Subassembly 


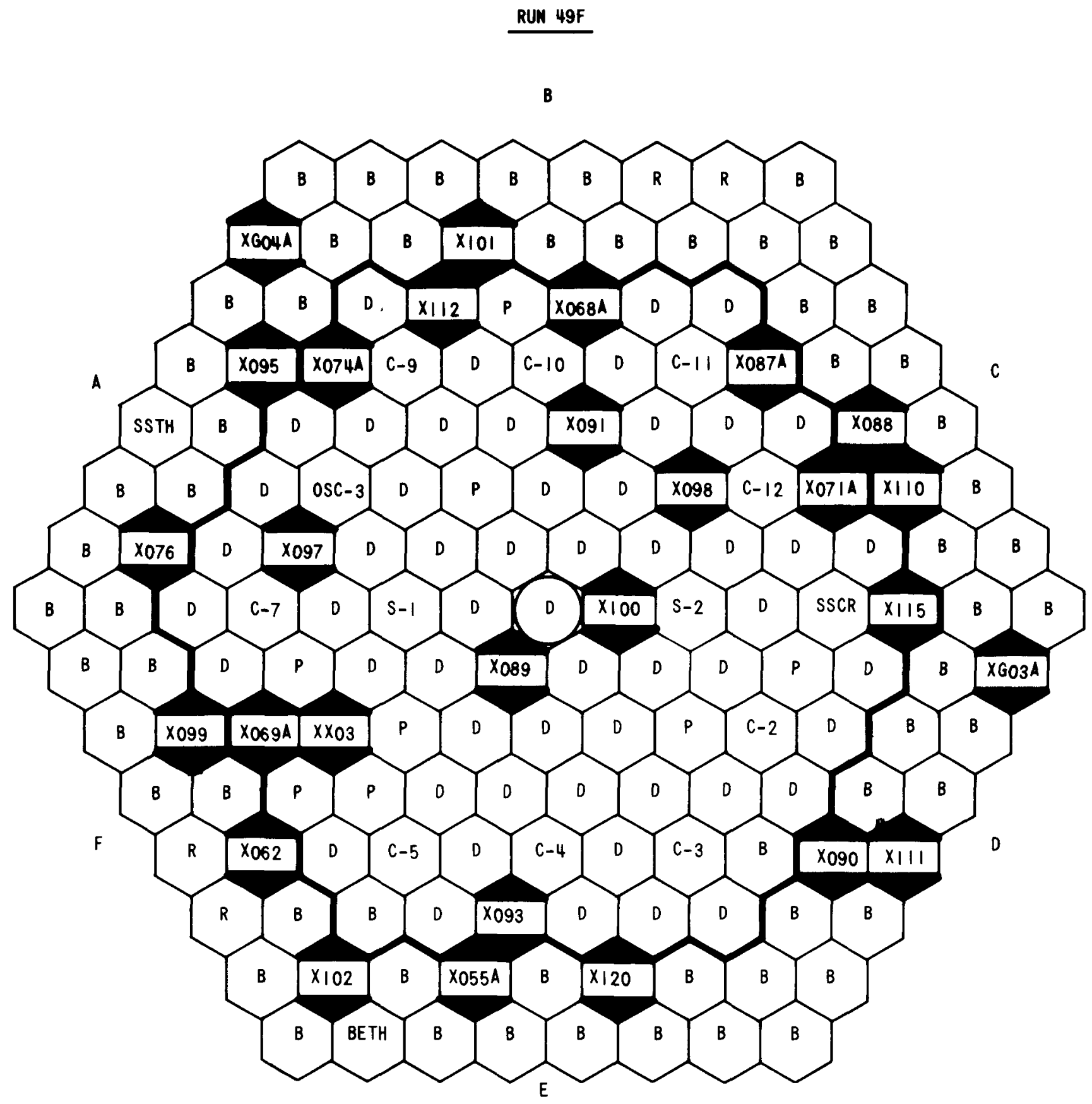




\section{RUN 50A}

(90-Subassemb1y Core Size)

\section{Chronology}

Date Started ............. 7/4/71

Date Ended .............. . . $7 / 5 / 71$

Number of Days for Run . . . . . . . . . . 1

Power Data

Cumulative MWd(t) at Start of Run . . . . 45,299

Cumulative MWd(t) at End of Run . . . . . 45,361

MWd $(t)$ Generated During Run ....... . 62

Types of Subassemblies in Core

Blanket ............. 20

Core ................ . . 39

Control Rod ............... 9

Safety Rod ............... 2

Experimentals (Rows 1-6) . . . . . . . 18

Experimental Subassemblies in Row 7 . . . . . 10

Key to Types of Subassemblies Shown in Loading Diagram

B -- Depleted Uranium

BETH -- Beryllium Thimble

C -- Contro1 Rod

D -- Driver Fuel

OSC -- Oscillator Rod

P -- 1/2 Driver Fue1 - 1/2 Stainless Steel

R -- Stainless Steel Reflector

$S$-- Safety Rod

SSCR -- Stainless Steel Control Rod

SSTH -- Stainless Steel Thimble

$\mathrm{X}$-- Experimental Subassembly 


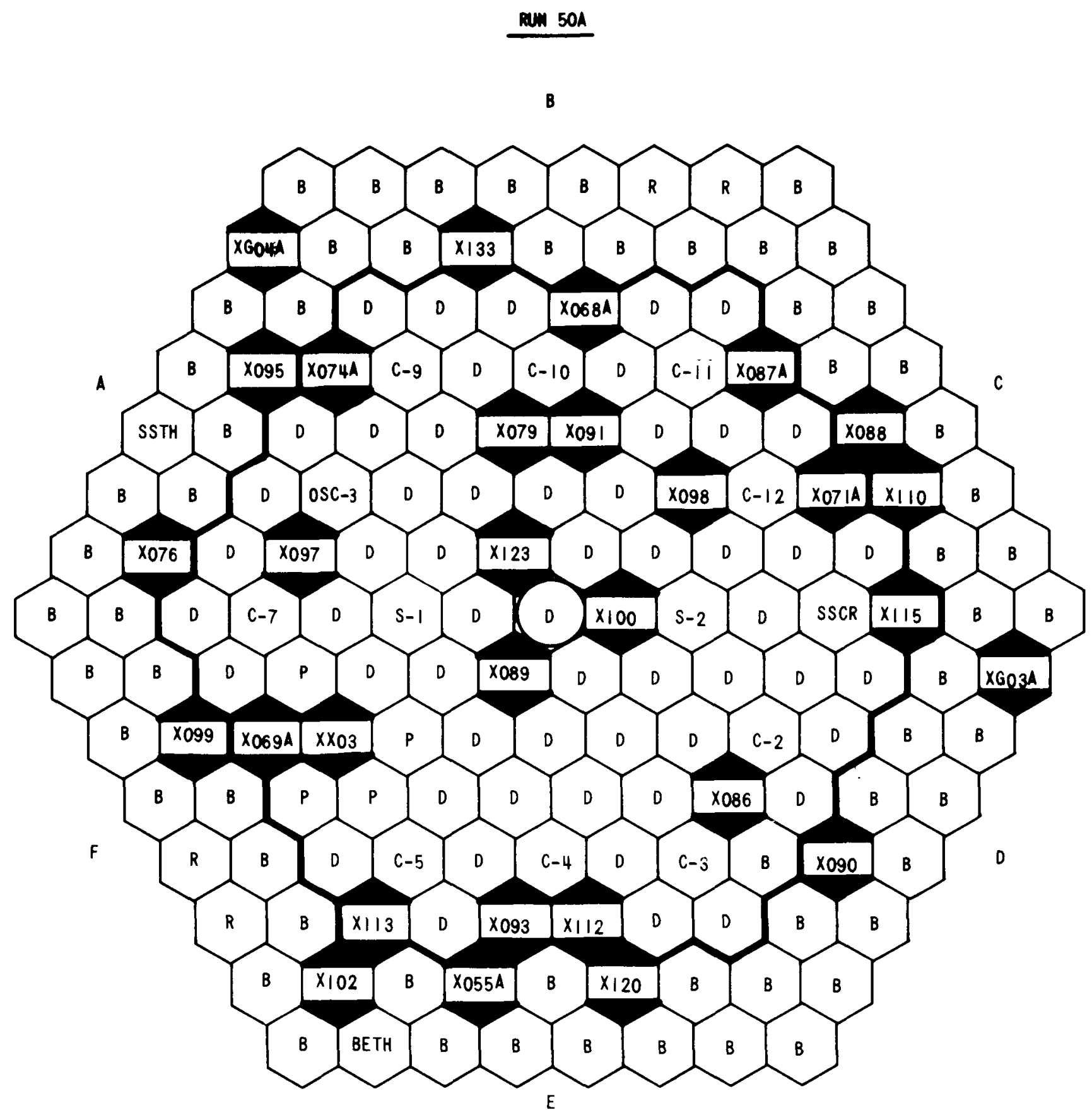


$\underline{\text { RUN 50B }}$

(89-Subassembly Core Size)

\section{Chronology}

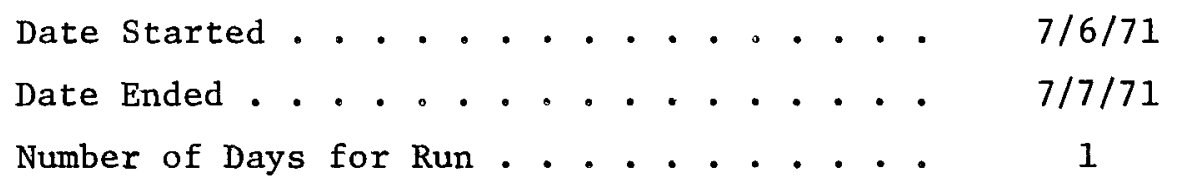

Power Data

Cumulative MWd(t) at Start of Run . . . . 45,361

Cumulative MWd(t) at End of Run . . . . 45,372

MWd $(t)$ Generated During Run . . . . . . 11

\section{Types of Subassemblies in Core}

Blanket ............ . . 20

Core ................. 40

Control Rod .............. . . 9

Safety Rod ............... 2

Experimentals (Rows 1-6) . . . . . . 16

Experimental Subassemblies in Row 7 . . . . . 13

Key to Types of Subassemblies Shown in Loading Diagram

B -- Depleted Uranium

BETH -- Beryllium Thimble

C -- Contro1 Rod

D -- Driver Fuel

OSC -- Oscillator Rod

P -- 1/2 Driver Fuel - 1/2 Stainless Steel

R -- Stainless Steel Reflector

S -- Safety Rod

SSCR -- Stainless Steel Control Rod

SSTH -- Stainless Steel Thimble

$X$-- Experimental Subassembly 


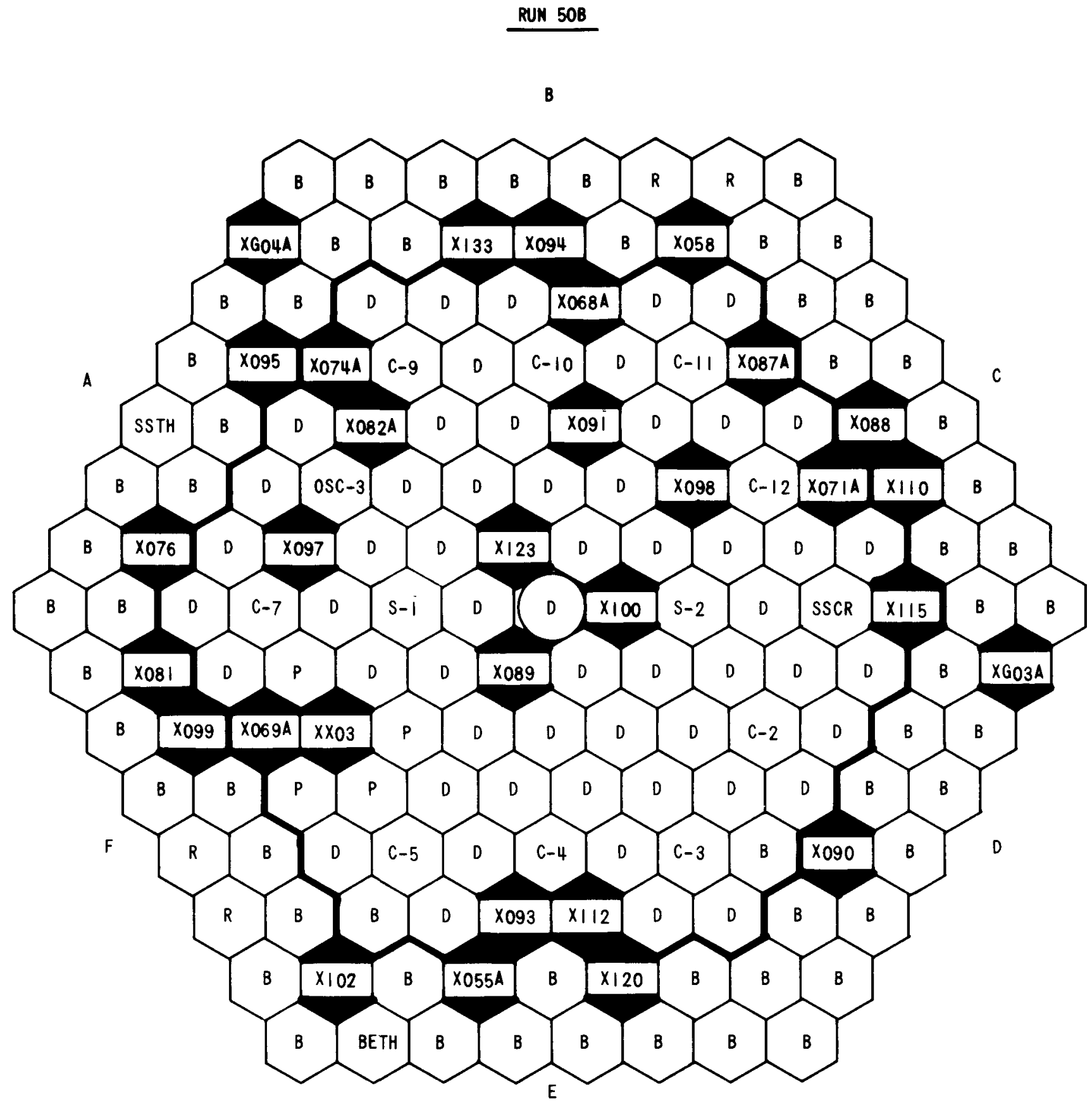


RUN 50C

(89-Subassembly Core Size)

Chronology

Date Started ............. . $7 / 8 / 71$

Date Ended ............. $7 / 11 / 71$

Number of Days for Run.......... . 3

Power Data

Cumulative MWd(t) at Start of Run . . . . 45,372

Cumulative MWd(t) at End of Run . . . . . 45,444

MWd $(t)$ Generated During Run . . . . . . 72

Types of Subassemblies in Core

Blanket . . . . . . . . . . . 20

Core ................ . . 41

Contro1 Rod .............. . . 9

Safety Rod . . . . . . . . . . . 2

Experimentals (Rows 1-6) . . . . . . . 15

Experimental Subassemblies in Row 7 . . . . . 12

Key to Types of Subassemblies Shown in Loading Diagram

B -- Depleted Uranium

BETH -- Beryllium Thimble

C -- Control Rod

D -- Driver Fuel

OSC -- Oscillator Rod

P -- 1/2 Driver Fuel - 1/2 Stainless Steel

R -- Stainless Steel Reflector

S -- Safety Rod

SSCR -- Stainless Steel Control Rod

SSTH -- Stainless Steel Thimble

$\mathrm{X}$-- Experimental Subassembly 
RUN $50 \mathrm{C}$

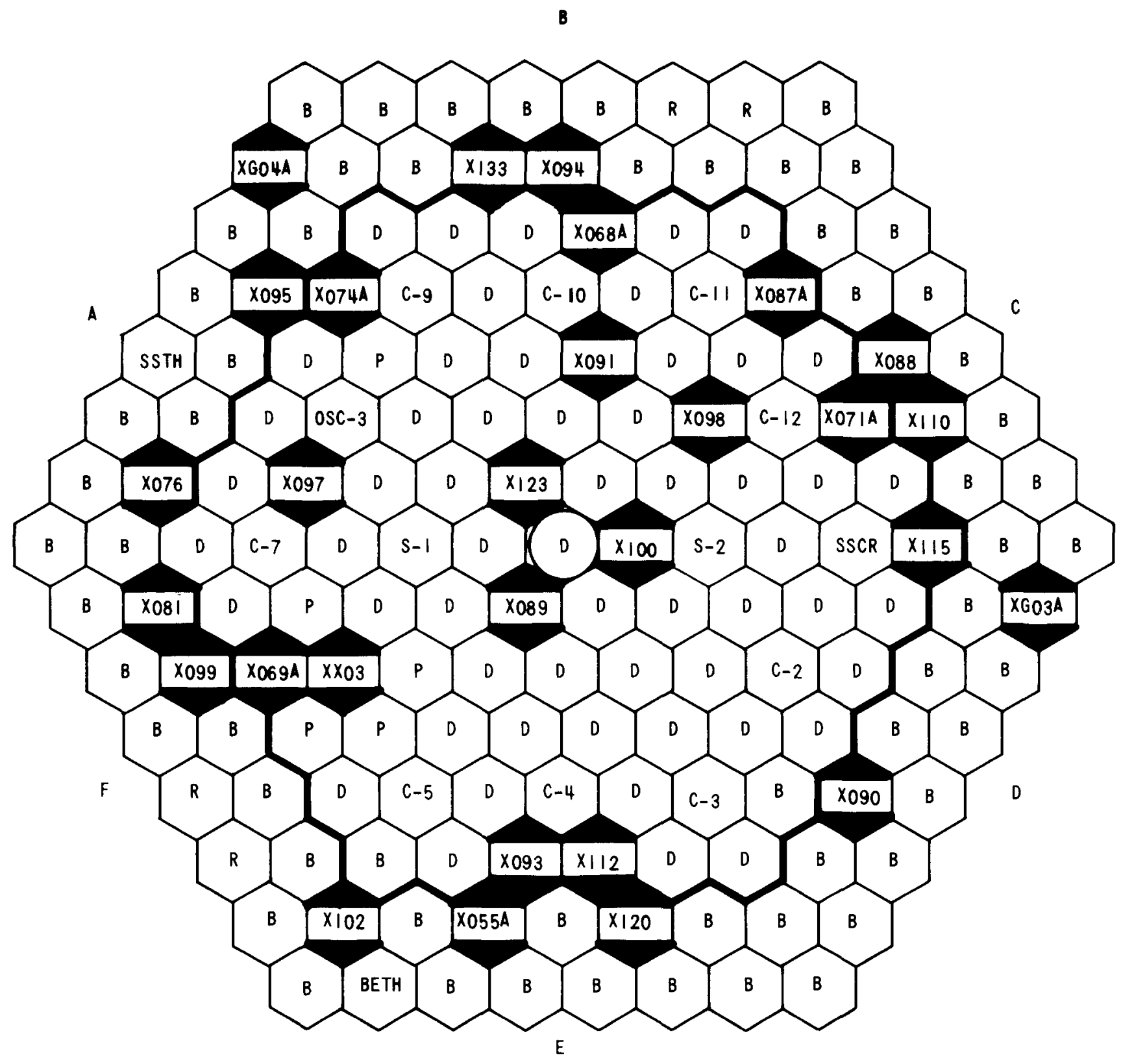




\section{Chronology}

Date Started ............. . $7 / 11 / 71$

Date Ended . . . . . . . . . . . 7/13/71

Number of Days for Run ........... 2

Power Data

Cumulative MWd(t) at Start of Run . . . . 45,444

Cumulative MWd(t) at End of Run ..... . 45,514

MWd $(t)$ Generated During Run ....... 70

Types of Subassemblies in Core

Blanket . . . . . . . . . . 20

Core . . . . . . . . . . . . 41

Control Rod .............. . 9

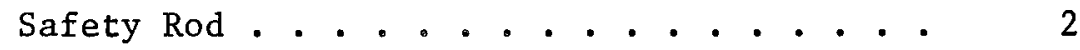

Experimentals (Rows 1-6) . . . . . . . 15

Experimenta1 Subassemblies in Row 7 . . . . . 11

Key to Types of Subassemb1ies. Shown in Loading Diagram

B -- Depleted Uranium

BETH -- Beryllium Thimble

C -- Control Rod

D -- Driver Fuel

OSC -- Oscillator Rod

P -- 1/2 Driver Fuel - 1/2 Stainless Stee1

R -- Stainless Steel Reflector

S -- Safety Rod

SSCR -- Stainless Steel Control Rod

SSTH -- Stainless Steel Thimble

$\mathrm{X}$-- Experimental Subassembly 


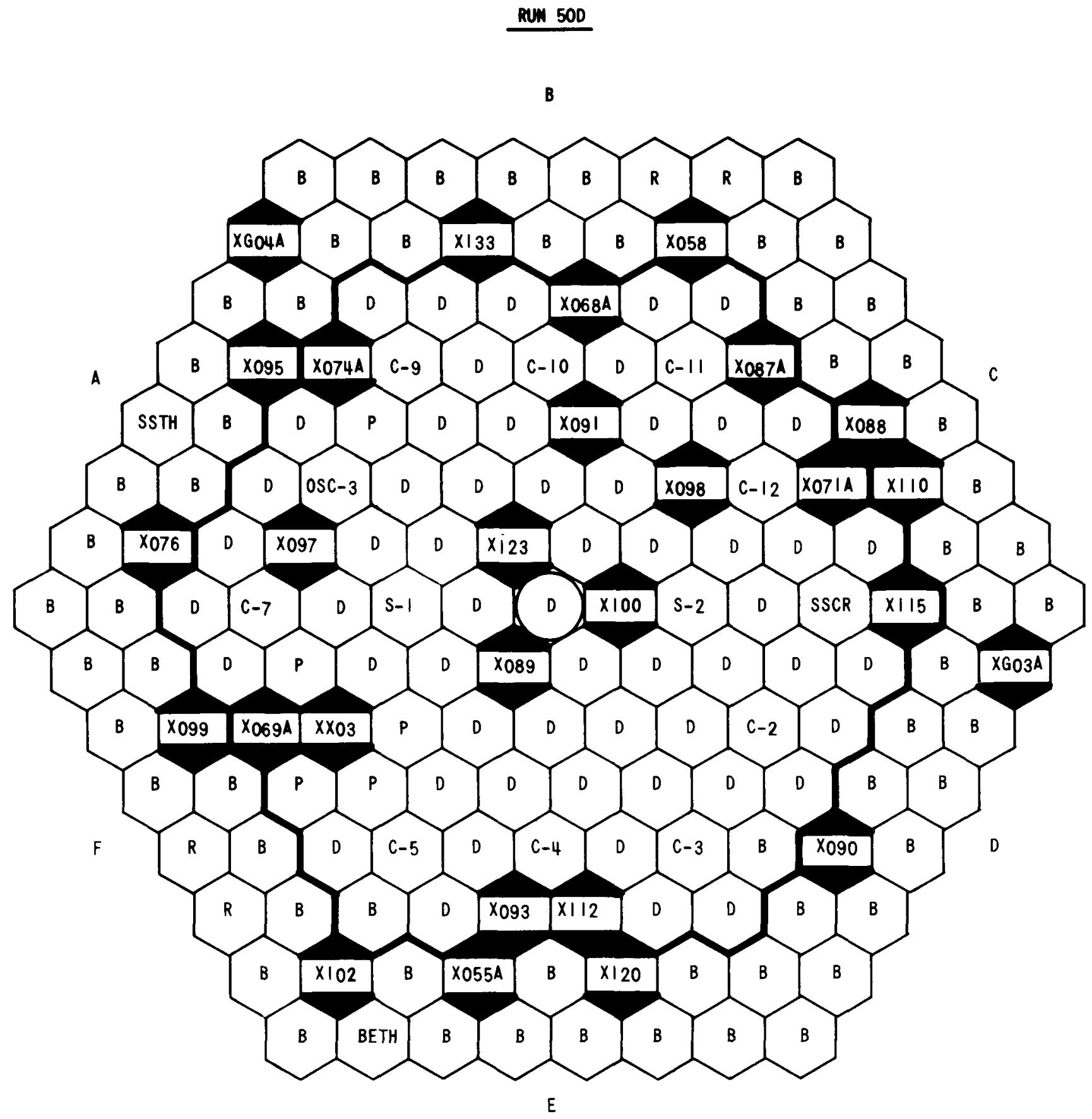


RUN 50E

(89-Subassemb1y Core Size)

Chronology

Date Started ............. . . $7 / 13 / 71$

Date Ended .............. . $7 / 14 / 71$

Number of Days for Run . . . . . . . . 1

Power Data

Cumulative MWd(t) at Start of Run . . . . 45,514

Cumulative MWd( $t$ ) at End of Run . . . . . 45,54Q

MWd $(t)$ Generated During Run ........ 26

Types of Subassemblies in Core

Blanket ............. 20

Core ................ 40

Control Rod .............. . 9

Safety Rod . . . . . . . . . . . . 2

Experimentals (Rows 1-6) ........ 16

Experimental Subassemblies in Row 7 . . . . . 10

Key to Types of Subassemblies Shown in Loading Diagram

B -- Depleted Uranium

BETH -- Bery1lium Thimble

C -- Control Rod

D -- Driver Fue1

OSC -- Oscillator Rod

P -- 1/2 Driver Fuel - 1/2 Stainless Steel

R -- Stainless Steel Reflector

S -- Safety Rod

SSCR -- Stainless Steel Control Rod

SSTH -- Stainless Steel Thimble

X -- Experimental Subassembly 
RUN 50E

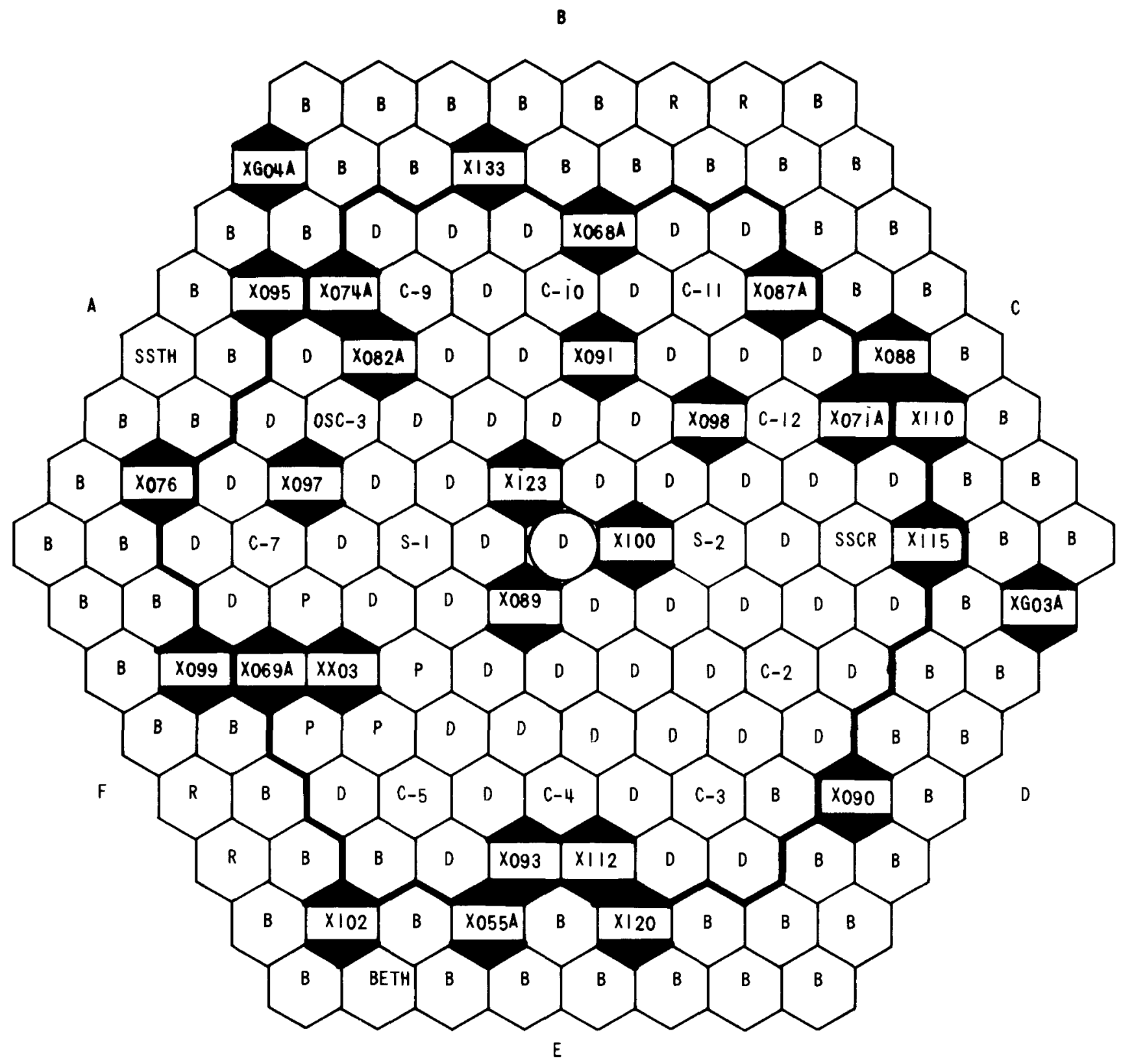


$\underline{\text { RUN 50F }}$

(89-Subassembly Core Size)

Chronology

Date Started ............. $7 / 16 / 71$

Date Ended . . . . . . . . . . . $7 / 29 / 71$

Number of Days for Run . . . . . . . . 13

\section{Power Data}

Cumulative MWd(t) at Start of Run . . . . 45,540

Cumulative MWd ( $t$ ) at End of Run ... . . 46,317

MWd $(t)$ Generated During Run . . . . . . 777

Types of Subassemblies in Core

Blanket . . . . . . . . . . 19

Core ............... . 40

Control Rod ............... 9

Safety Rod ............... 2

Experimentals (Rows 1-6) . . . . . . . . 17

Experimental Subassemblies in Row 7 . . . . . . 13

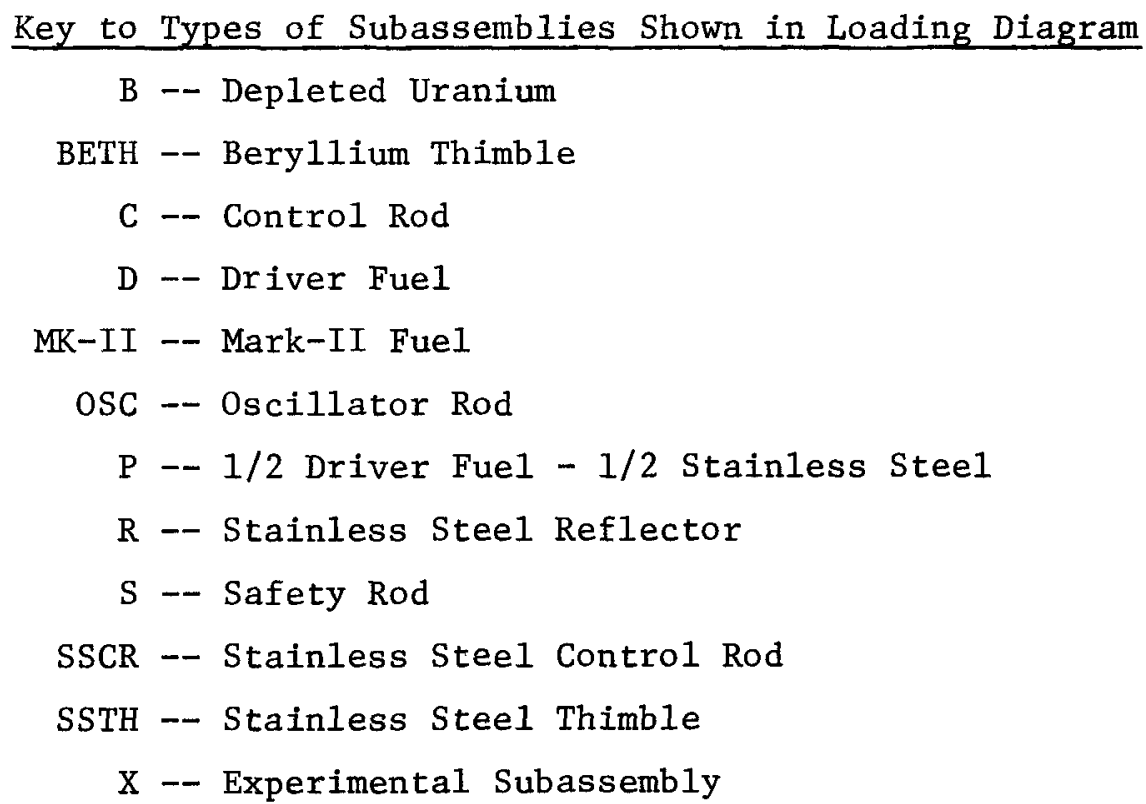




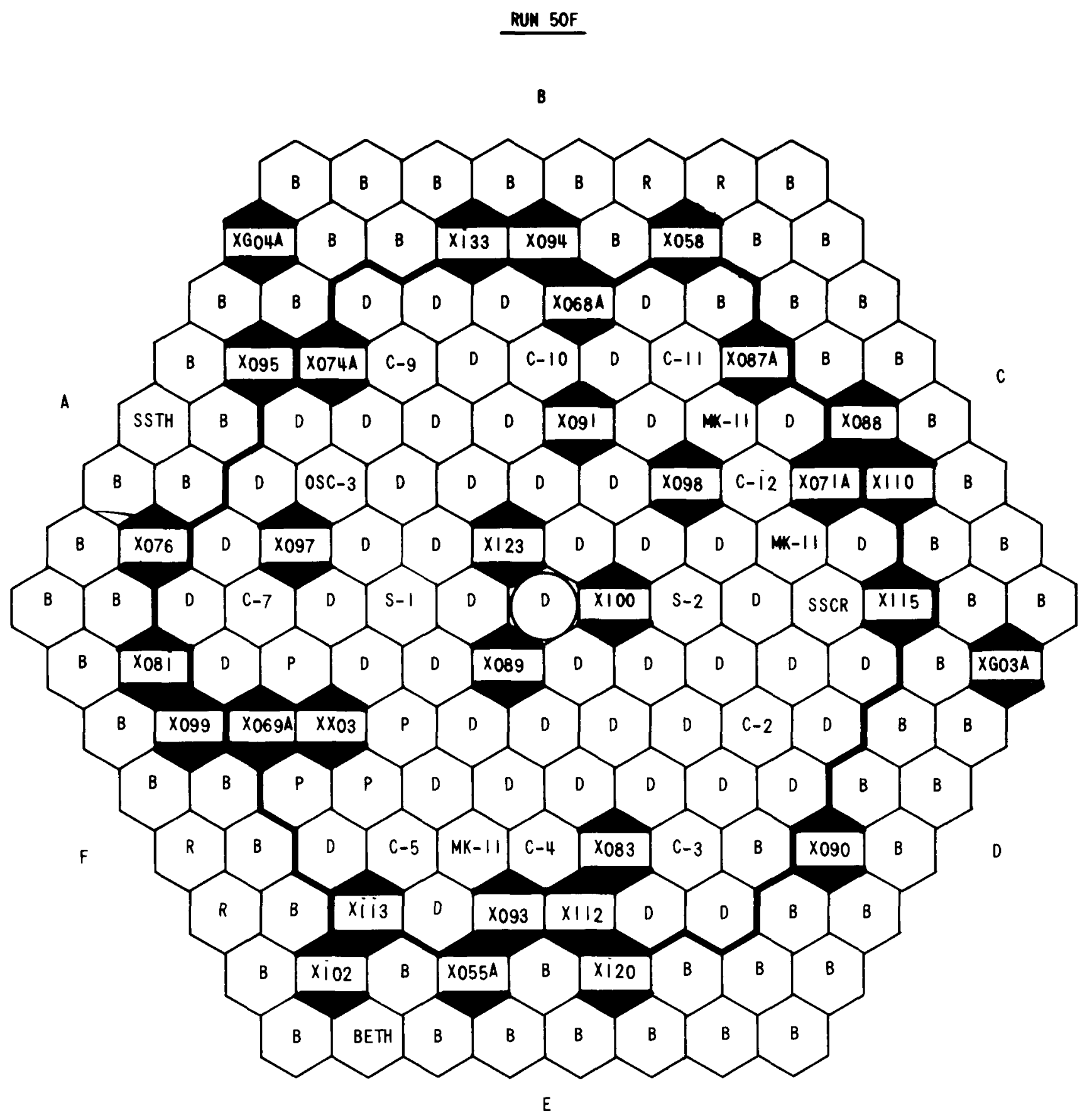


RUN 50G

(90-Subassemb1y Core Size)

\section{Chronology \\ Date Started .............. . $8 / 3 / 71$ \\ Date Ended .............. 8/3/71 \\ Number of Days for Run .......... $1 \mathrm{hr}$}

\section{Power Data}

Cumulative MWd(t) at Start of Run . . . . 46,317

Cumulative MWd(t) at End of Run . . . . . 46,317

MWd $(t)$ Generated During Run . . . . . . 0

\section{Types of Subassemblies in Core}

Blanket ............. 16

Core ................. 33

Control Rod . . . . . . . . . . 9

Safety Rod . . . . . . . . . . . . 2

Experimentals (Rows 1-6) . . . . . . . 21

Experimental Subassemblies in Row 7 . . . . 13

Key to Types of Subassemblies Shown in Loading Diagram

B -- Depleted Uranium

BETH -- Beryllium Thimble

C -- Control Rod

D -- Driver Fue1

MK-II -- Mark-II Fuel

OSC -- Oscillator Rod

P -- 1/2 Driver Fuel - 1/2 Stainless Steel

R -- Stainless Steel Reflector

S -- Safety Rod

SSCR -- Stainless Steel Control Rod

SSTH -- Stainless Steel Thimble

W -- F1ux-wire Subassembly

$\mathrm{X}$-- Experimental Subassembly 
RUN 506

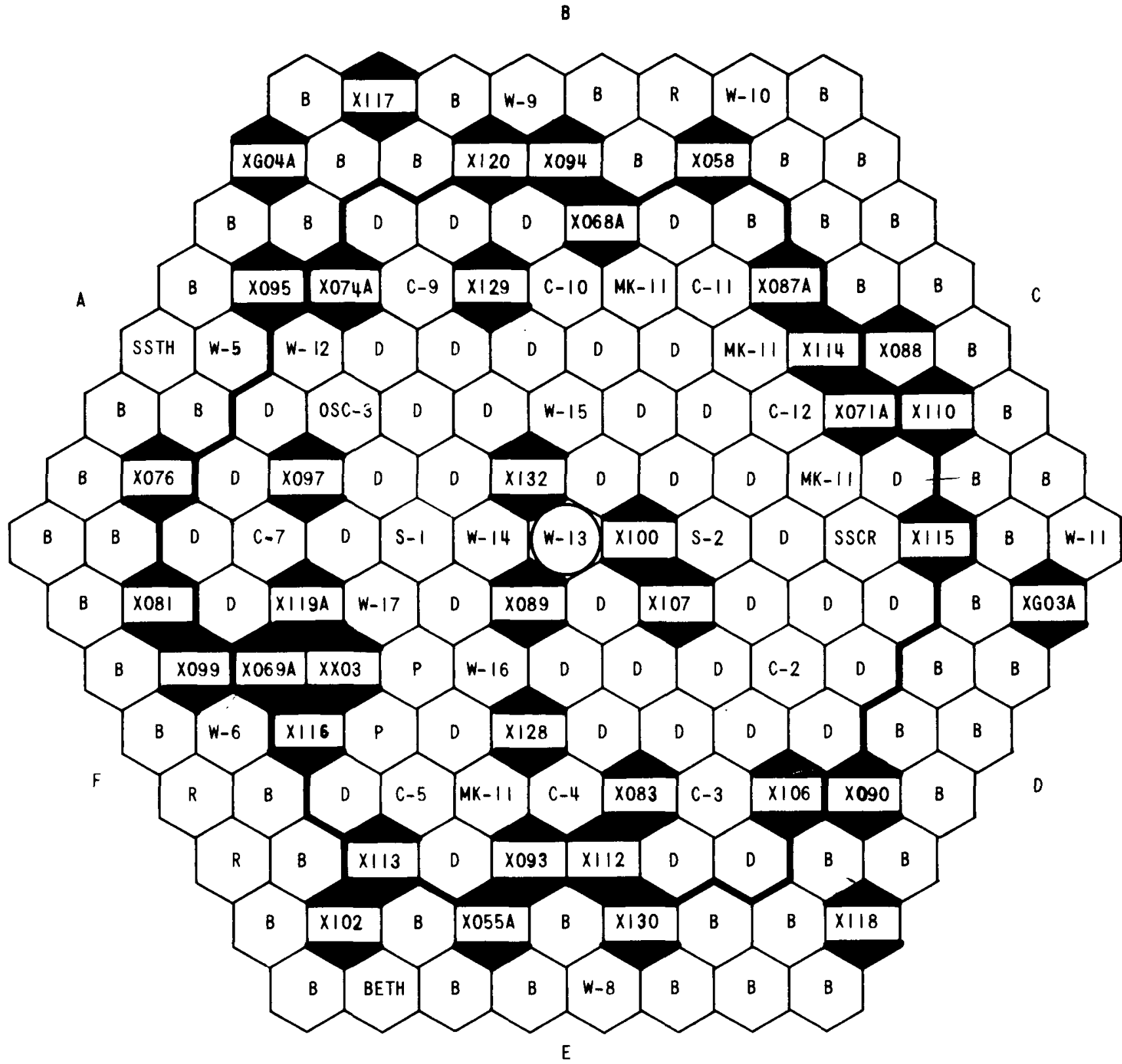


RUN $50 \mathrm{H}$

(90-Subassemb1y Core Size)

Chronology

Date Started............. $8 / 6 / 71$

Date Ended ............ . . 8/15/71

Number of Days for Run ......... . . 9

Power Data

Cumulative MWd(t) at Start of Run . . . . 46,317

Cumulative MWd( $t$ ) at End of Run . . . . . 46,863

MWd $(t)$ Generated During Run . . . . . . 546

Types of Subassemblies in Core

Blanket . . . . . . . . . . 17

Core ................ . 37

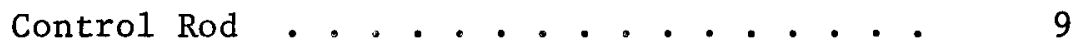

Safety Rod .............. . 2

Experimentals (Rows 1-6)......... . 23

Experimental Subassemblies in Row 7 . . . . 13

Key to Types of Subassemblies Shown in Loading Diagram

B -- Depleted Uranium

BETH -- Beryllium Thimble

C -- Control Rod

D -- Driver Fuel

MK-II -- Mark-II Fue1

OSC -- Oscillator Rod

P -- 1/2 Driver Fuel - 1/2 Stainless Steel

R -- Stainless Steel Reflector

S -- Safety Rod

SSCR -- Stainless Steel Control Rod

SSTH -- Stainless Steel Thimble

$X$-- Experimental Subassembly 
RLN 50H

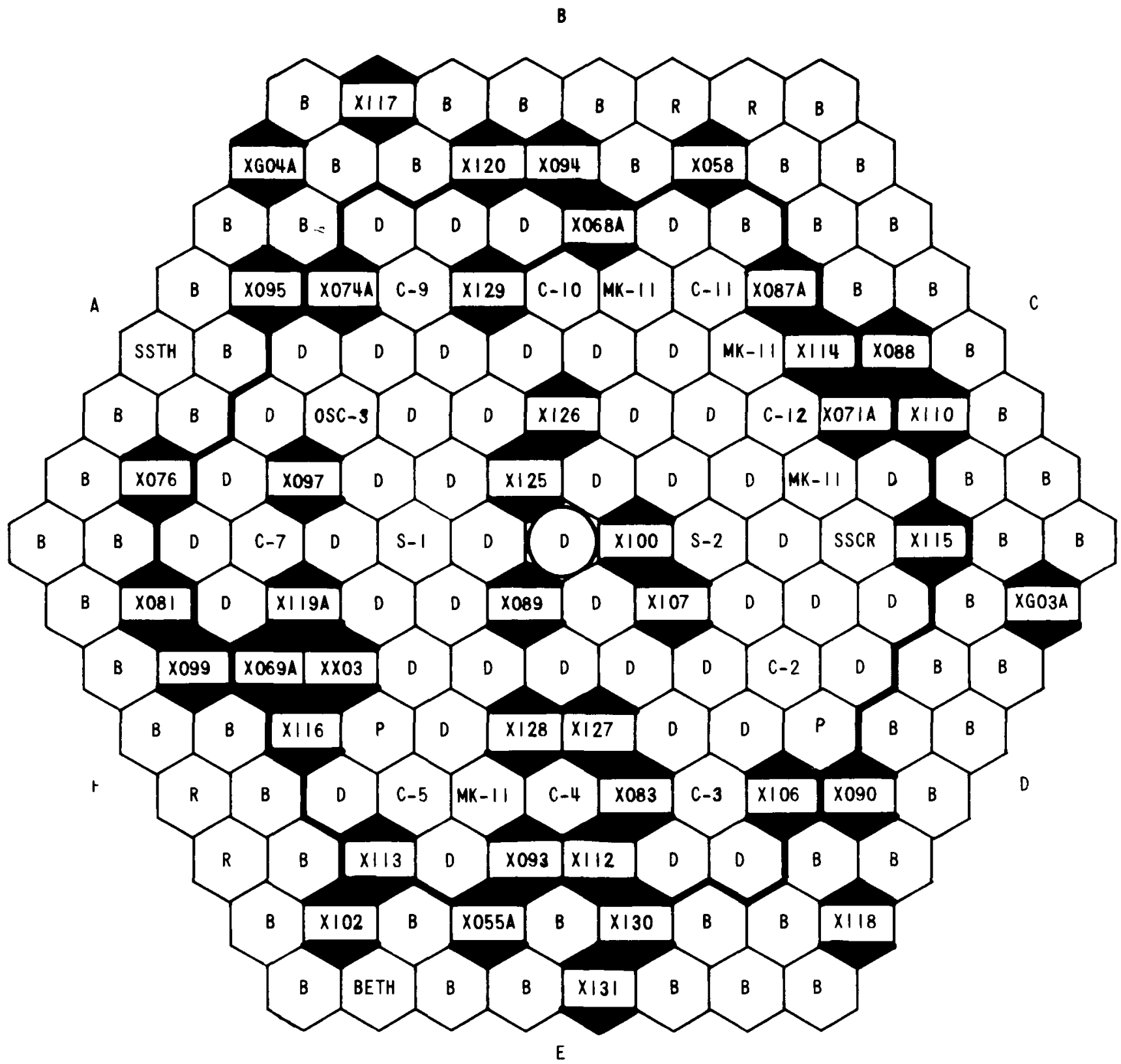


- $76-$

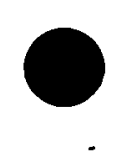


EBR-II Power-run Information: Runs 1-50

\begin{tabular}{|c|c|c|c|c|c|c|c|c|c|c|c|c|c|}
\hline \multirow{2}{*}{$\begin{array}{l}\text { Run } \\
\text { No. }\end{array}$} & \multicolumn{3}{|c|}{ Megawatt-Days } & \multicolumn{3}{|c|}{ Date } & \multirow{2}{*}{$\begin{array}{l}\text { Core } \\
\text { Size }\end{array}$} & \multicolumn{4}{|c|}{ Subassemb1y Type } & \multicolumn{2}{|c|}{ Experimentals } \\
\hline & Start & Stop & Incr. & Start & Stop & Days & & B & $\mathrm{C}$ & $\mathrm{L}$ & $\mathrm{S}$ & Rows $1-6$ & Row 7 \\
\hline 1 & 0 & 343 & 343 & $8 / 1 / 64$ & $8 / 23 / 64$ & 22 & 73 & 12 & -.7 & 11 & 2 & & \\
\hline 2 & 343 & 705 & 362 & $10 / 2 / 64$ & $10 / 19 / 64$ & 17 & 73 & 12 & 47 & 11 & 2 & & \\
\hline 3 & 705 & 1,280 & 575 & $3 / 15 / 65$ & $4 / 10 / 65$ & $\angle 6$ & 72 & 11. & 47 & 12 & 2 & & \\
\hline 4 & 1,280 & 1,631 & 351 & $4 / 24 / 65$ & $5 / 4 / 65$ & 10 & 72 & 11 & 47 & 12 & 2 & & \\
\hline 5 & 1,631 & 2,003 & 372 & $5 / 10 / 65$ & $5 / 21 / 65$ & 11 & 76 & 14 & 46 & 12 & 2 & 2 & \\
\hline 6 & 2,003 & 2,502 & 499 & $5 / 24 / 65$ & $6 / 25 / 65$ & 32 & 75 & 13 & 47 & 11 & 2 & 1 & \\
\hline 7 & 2,502 & 2,927 & 425 & $7 / 29 / 65$ & $8 / 15 / 65$ & 17 & 74 & 12 & 47 & 11 & 2 & 1 & 3 \\
\hline 8 & 2,927 & 3,320 & 393 & $9 / 7 / 65$ & $9 / 19 / 65$ & 12 & 74 & 12 & 47 & 11 & 2 & 1 & 3 \\
\hline 9 & 3,320 & 3,665 & 345 & $10 / 13 / 65$ & $10 / 21 / 65$ & 8 & 76 & 15 & 46 & 11 & 2 & 3 & 3 \\
\hline 10 & 3,665 & 3,980 & 315 & $10 / 28 / 65$ & $11 / 7 / 65$ & 10 & 79 & 17 & 44 & 11 & 2 & 4 & 3 \\
\hline 11 & 3,980 & 4,280 & 300 & $11 / 18 / 65$ & $11 / 27 / 65$ & 9 & 79 & 17 & 44 & 11 & 2 & 4 & 3 \\
\hline 12 & 4,280 & 4,750 & 470 & $11 / 30 / 65$ & $12 / 11 / 65$ & 11 & 78 & 16 & 44 & 11 & 2 & 4 & 3 \\
\hline 13 & 4,750 & 5,050 & 300 & $12 / 17 / 65$ & $12 / 24 / 65$ & 7 & 80 & 18 & 43 & 11 & 2 & 5 & 3 \\
\hline
\end{tabular}


EBR-II Power-run Information: Runs 1-50 (contd)

\begin{tabular}{|c|c|c|c|c|c|c|c|c|c|c|c|c|c|}
\hline \multirow{2}{*}{$\begin{array}{l}\text { Run } \\
\text { No. }\end{array}$} & \multicolumn{3}{|c|}{ Megawatt-Days } & \multicolumn{3}{|c|}{ Date } & \multirow{2}{*}{$\begin{array}{l}\text { Core } \\
\text { Size } \\
\end{array}$} & \multicolumn{4}{|c|}{ Subassembly Type } & \multicolumn{2}{|c|}{ Experimentals } \\
\hline & Start & Stop & Incr. & Start & Stop & Days & & B & $\mathrm{C}$ & $\mathrm{L}$ & $S$ & Rows $1-6$ & Row 7 \\
\hline 14 & 5,050 & 5,570 & 520 & $1 / 10 / 66$ & $1 / 22 / 66$ & 12 & 80 & 18 & 43 & 11 & 2 & 5 & 3 \\
\hline 15 & 5,570 & 6,365 & 795 & $3 / 28 / 66$ & $4 / 14 / 66$ & 17 & 77 & 16 & 42 & 11 & 2 & 5 & 4 \\
\hline 16 & 6,365 & 7,140 & 775 & $4 / 17 / 66$ & $5 / 8 / 66$ & 21 & 75 & 14 & 42 & 11 & 2 & 5 & 4 \\
\hline 17 & 7,140 & 7,615 & 475 & $5 / 11 / 66$ & $5 / 23 / 66$ & 12 & 77 & 16 & 41 & 11 & 2 & 6 & 4 \\
\hline 18 & 7,615 & 8,482 & 867 & $5 / 26 / 66$ & $6 / 15 / 66$ & 20 & 76 & 15 & 41 & 11 & 2 & 6 & 4 \\
\hline 19 & 8,482 & 8,571 & 89 & $6 / 27 / 66$ & $6 / 29 / 66$ & 2 & 74 & 13 & 41 & 11 & 2 & 6 & 4 \\
\hline 20 & 8,571 & 9,270 & 699 & $7 / 19 / 66$ & $8 / 7 / 66$ & 19 & 80 & 19 & 39 & 11 & 2 & 8 & 4 \\
\hline 21 & 9,270 & 9,880 & 610 & $8 / 12 / 66$ & $8 / 27 / 66$ & 15 & 80 & 19 & 38 & 11 & 2 & 9 & 4 \\
\hline 22 & 9,880 & 10,925 & 1,045 & $10 / 20 / 66$ & $11 / 14 / 66$ & 25 & 78 & 17 & 39 & 11 & 2 & 8 & 4 \\
\hline 23 & 10,925 & 11,615 & 690 & $11 / 16 / 66$ & $12 / 3 / 66$ & 17 & 79 & 18 & 38 & 11 & 2 & 9 & 4 \\
\hline 24 & 11,615 & 12,245 & 630 & $12 / 6 / 66$ & $12 / 31 / 66$ & 25 & 81 & 20 & 38 & 11 & 2 & 9 & 4 \\
\hline 25 & 12,245 & 13,806 & 1,561 & $4 / 17 / 67$ & $8 / 17 / 67$ & 122 & 88 & 25 & 37 & 11 & 2 & 11 & 5 \\
\hline $26 \mathrm{~A}$ & 13,806 & 13,893 & 87 & $9 / 22 / 67$ & $9 / 29 / 67$ & 7 & 88 & 25 & 38 & 11 & 2 & 10 & 5 \\
\hline
\end{tabular}




\section{EBR-II Power-run Information: Runs 1-50 (contd)}

\begin{tabular}{|c|c|c|c|c|c|c|c|c|c|c|c|c|c|}
\hline \multirow{2}{*}{$\begin{array}{l}\text { Run } \\
\text { No. }\end{array}$} & \multicolumn{3}{|c|}{ Megawatt-Days } & \multicolumn{3}{|c|}{ Date } & \multirow{2}{*}{$\begin{array}{l}\text { Core } \\
\text { Size }\end{array}$} & \multicolumn{4}{|c|}{ Subassembly Type } & \multicolumn{2}{|c|}{ Experimentals } \\
\hline & Start & Stop & Incr. & Start & Stop & Days & & $\mathrm{B}$ & $\mathrm{C}$ & $\mathrm{L}$ & $S$ & Rows $1-6$ & Row 7 \\
\hline $26 B$ & 13,893 & 14,922 & 1,029 & $10 / 11 / 67$ & $11 / 20 / 67$ & 40 & 91 & 27 & 38 & 10 & 2 & 12 & 6 \\
\hline $26 \mathrm{C}$ & 14,922 & 15,541 & 619 & $11 / 22 / 67$ & $12 / 12 / 67$ & 21 & 91 & 25 & 36 & 10 & 2 & 16 & 6 \\
\hline $27 \mathrm{~A}$ & 15,541 & 15,824 & 283 & $2 / 2 / 68$ & $2 / 29 / 68$ & 27 & 91 & 25 & 36 & 10 & 2 & 16 & 6 \\
\hline $27 \mathrm{~B}$ & 15,824 & 16,001 & 177 & $3 / 1 / 68$ & $3 / 5 / 68$ & 4 & 91 & 25 & 35 & 10 & 2 & 17 & 6 \\
\hline $27 \mathrm{C}$ & 16,001 & 16,063 & 62 & $3 / 7 / 68$ & $3 / 11 / 68$ & 4 & 90 & 25 & 35 & 10 & 2 & 16 & 6 \\
\hline $27 D$ & 16,063 & 16,349 & 286 & $3 / 30 / 68$ & $4 / 6 / 68$ & 7 & 90 & 25 & 36 & 10 & 2 & 15 & 7 \\
\hline $27 \mathrm{E}$ & 16,349 & 16,439 & 90 & $4 / 7 / 68$ & $4 / 11 / 68$ & 4 & 87 & 22 & 39 & 10 & 2 & 12 & 7 \\
\hline $27 \mathrm{~F}$ & 16,439 & 16,507 & 68 & $4 / 13 / 68$ & $4 / 16 / 68$ & 3 & 86 & 24 & 39 & 10 & 2 & 9 & 8 \\
\hline $27 G$ & 16,507 & 16,556 & 49 & $4 / 17 / 68$ & $4 / 19 / 68$ & 2 & 86 & 25 & 40 & 10 & 2 & 7 & 4 \\
\hline $27 \mathrm{H}$ & 16,556 & 16,762 & 206 & $4 / 25 / 68$ & $5 / 2 / 68$ & 7 & 83 & 22 & 42 & 10 & 2 & 5 & 4 \\
\hline $27 I$ & 16,762 & 16,858 & 96 & $5 / 3 / 68$ & $5 / 6 / 68$ & 3 & 85 & 24 & 41 & 10 & 2 & 6 & 4 \\
\hline $28 \mathrm{~A}$ & 16,858 & 17,012 & 154 & $5 / 9 / 68$ & $5 / 13 / 68$ & 4 & 86 & 25 & 41 & 10 & 2 & 6 & 6 \\
\hline $28 \mathrm{~B}$ & 17,012 & 17,315 & 303 & $5 / 15 / 68$ & $5 / 27 / 68$ & 12 & 88 & 23 & 41 & 10 & 2 & 10 & 10 \\
\hline
\end{tabular}


EBR-II Power-run Information: Runs 1-50 (contd)

\begin{tabular}{|c|c|c|c|c|c|c|c|c|c|c|c|c|c|}
\hline \multirow{2}{*}{$\begin{array}{l}\text { Run } \\
\text { No. }\end{array}$} & \multicolumn{3}{|c|}{ Megawatt-Days } & \multicolumn{3}{|c|}{ Date } & \multirow{2}{*}{$\begin{array}{l}\text { Core } \\
\text { Size }\end{array}$} & \multicolumn{4}{|c|}{ Subassembly Type } & \multicolumn{2}{|c|}{ Experimentals } \\
\hline & Start & Stop & Incr. & Start & Stop & Days & & $\mathrm{B}$ & $\mathrm{C}$ & $\mathrm{L}$ & $\mathrm{S}$ & Rows $1-6$ & Row 7 \\
\hline $28 \mathrm{C}$ & 17,315 & 17,984 & 669 & $5 / 29 / 68$ & $6 / 15 / 68$ & 17 & 88 & 23 & 35 & 10 & 2 & 16 & 10 \\
\hline $29 A$ & 17,984 & 18,172 & 188 & $6 / 26 / 68$ & $7 / 5 / 68$ & 9 & 87 & 23 & 35 & 11 & 2 & 15 & 5 \\
\hline $29 B$ & 18,172 & 18,196 & 24 & $7 / 9 / 68$ & $7 / 11 / 68$ & 2 & 89 & 25 & 35 & 11 & 2 & 15 & 5 \\
\hline $29 \mathrm{C}$ & 18,196 & 18,401 & 205 & $7 / 15 / 68$ & $7 / 22 / 68$ & 7 & 91 & 27 & 35 & 11 & 2 & 15 & 5 \\
\hline $29 D$ & 18,401 & 19,111 & 710 & $7 / 25 / 68$ & $8 / 16 / 68$ & 22 & 91 & 27 & 35 & 11 & 2 & 15 & 11 \\
\hline $30 \mathrm{~A}$ & 19,111 & 19,763 & 652 & $8 / 21 / 68$ & $9 / 5 / 68$ & 15 & 91 & 27 & 34 & 11 & 2 & 16 & 9 \\
\hline $30 \mathrm{~B}$ & 19,763 & 19,929 & 166 & $9 / 7 / 68$ & $9 / 11 / 68$ & 4 & 91 & 27 & 34 & 11 & 2 & 16 & 9 \\
\hline $30 \mathrm{C}$ & 19,929 & 19,981 & 52 & $9 / 11 / 68$ & $9 / 15 / 68$ & 4 & 91 & 27 & 35 & 11 & 2 & 15 & 9 \\
\hline $30 D$ & 19,981 & 20,060 & 79 & $9 / 17 / 68$ & $9 / 21 / 68$ & 4 & 90 & 28 & 40 & 11 & 2 & 8 & 6 \\
\hline $30 \mathrm{E}$ & 20,060 & 20,139 & 79 & $9 / 22 / 68$ & $9 / 25 / 68$ & 3 & 89 & 28 & 42 & 11 & 2 & 5 & 5 \\
\hline $31 \mathrm{~A}$ & 20,139 & 20,228 & 89 & $9 / 30 / 68$ & $10 / 4 / 68$ & 4 & 90 & 26 & 36 & 11 & 2 & 14 & 9 \\
\hline $31 B$ & 20,228 & 20,387 & 159 & $10 / 11 / 68$ & $10 / 18 / 68$ & 7 & 90 & 26 & 37 & 11 & 2 & 13 & 9 \\
\hline $31 C$ & 20,387 & 20,471 & 84 & $10 / 19 / 68$ & $10 / 22 / 68$ & 3 & 90 & 26 & 37 & 11 & 2 & 13 & 9 \\
\hline
\end{tabular}


EBR-II Power-run Information: Runs $1-50$ (contd)

\begin{tabular}{|c|c|c|c|c|c|c|c|c|c|c|c|c|c|}
\hline \multirow{2}{*}{$\begin{array}{l}\text { Run } \\
\text { No. }\end{array}$} & \multicolumn{3}{|c|}{ Megawatt-Days } & \multicolumn{3}{|c|}{ Date } & \multirow{2}{*}{$\begin{array}{l}\text { Core } \\
\text { Size }\end{array}$} & \multicolumn{4}{|c|}{ Subassemb1y Type } & \multicolumn{2}{|c|}{ Experimentals } \\
\hline & Start & Stop & Incr. & Start & Stop & Days & & B & $\mathrm{C}$ & $\mathrm{L}$ & $\mathrm{S}$ & Rows $1-6$ & Row 7 \\
\hline $31 D$ & 20,471 & 20,843 & 372 & $10 / 26 / 68$ & $11 / 3 / 68$ & 8 & 90 & 26 & 37 & 11 & 2 & 13 & 9 \\
\hline $31 E$ & 20,843 & 20,843 & 0 & $11 / 4 / 68$ & $11 / 4 / 68$ & $1 \mathrm{hr}$ & 90 & 26 & 36 & 11 & 2 & 14 & 9 \\
\hline $31 F$ & 20,843 & 21,256 & 413 & $11 / 5 / 68$ & $11 / 14 / 68$ & 9 & 90 & 26 & 36 & 11 & 2 & 14 & 9 \\
\hline $31 G$ & 21,256 & 21,367 & 111 & $11 / 15 / 68$ & $11 / 17 / 68$ & 2 & 90 & 26 & 36 & 12 & 2 & 14 & 9 \\
\hline $32 \mathrm{~A}$ & 12,367 & 22,219 & 852 & $11 / 27 / 68$ & $12 / 17 / 68$ & 20 & 90 & 26 & 34 & 11 & 2 & 16 & 9 \\
\hline $32 B$ & 22,219 & 22,468 & 249 & $12 / 18 / 68$ & $12 / 24 / 68$ & 6 & 90 & 26 & 34 & 11 & 2 & 16 & 9 \\
\hline $32 \mathrm{C}$ & 22,468 & 22,540 & 72 & $12 / 25 / 68$ & $12 / 27 / 68$ & 2 & 90 & 26 & 35 & 11 & 2 & 15 & 9 \\
\hline $32 D$ & 22,540 & 22,764 & 224 & $12 / 28 / 68$ & $1 / 2 / 69$ & 5 & 89 & 27 & 37 & 11 & 2 & 11 & 9 \\
\hline $33 \mathrm{~A}$ & 22,764 & 23,364 & 600 & $2 / 25 / 69$ & $3 / 29 / 69$ & 32 & 90 & 25 & 33 & 11 & 2 & 18 & 8 \\
\hline $33 B$ & 23,364 & 23,940 & 576 & $4 / 7 / 69$ & $4 / 22 / 69$ & 15 & 91 & 26 & 32 & 11 & 2 & 19 & 10 \\
\hline $34 \mathrm{~A}$ & 23,940 & 24,762 & 822 & $4 / 25 / 69$ & $5 / 13 / 69$ & 18 & 91 & 26 & 30 & 11 & 2 & 21 & 11 \\
\hline $34 \mathrm{~B}$ & 24,762 & 25,072 & 310 & $5 / 14 / 69$ & $5 / 21 / 69$ & 7 & 91 & 26 & 30 & 11 & 2 & 21 & 11 \\
\hline 35 & 25,072 & 26,274 & 1,202 & $5 / 29 / 69$ & $6 / 26 / 69$ & 28 & 91 & 25 & 30 & 11 & 2 & 22 & 11 \\
\hline
\end{tabular}


EBR-II Power-run Information: Runs 1-50 (contd)

\begin{tabular}{|c|c|c|c|c|c|c|c|c|c|c|c|c|c|}
\hline \multirow{2}{*}{$\begin{array}{l}\text { Run } \\
\text { No. }\end{array}$} & \multicolumn{3}{|c|}{ Megawatt-Days } & \multicolumn{3}{|c|}{ Date } & \multirow{2}{*}{$\begin{array}{l}\text { Core } \\
\text { Size }\end{array}$} & \multicolumn{4}{|c|}{ Subassembly Type } & \multicolumn{2}{|c|}{ Experimentals } \\
\hline & Start & Stop & Incr. & Start & Stop & Days & & $B$ & $\mathrm{C}$ & $\mathrm{L}$ & $s$ & Rows $1-6$ & Row 7 \\
\hline $36 \mathrm{~A}$ & 26,274 & 27,074 & 800 & $6 / 30 / 69$ & $7 / 20 / 69$ & 20 & 91 & 24 & 32 & 11 & 2 & 21 & 12 \\
\hline $36 \mathrm{~B}$ & 27,074 & 27,474 & 400 & $7 / 22 / 69$ & $7 / 31 / 69$ & 9 & 91 & 24 & 32 & 11 & 2 & 21 & 12 \\
\hline 37 & 27,474 & 28,670 & 1,196 & $8 / 2 / 69$ & $8 / 30 / 69$ & 28 & 89 & 23 & 34 & 11 & 2 & 18 & 10 \\
\hline $38 \mathrm{~A}$ & 28,670 & 29,440 & 770 & $9 / 10 / 69$ & $9 / 28 / 69$ & 18 & 89 & 28 & 42 & 11 & 2 & 5 & 7 \\
\hline $38 \mathrm{~B}$ & 29,440 & 30,040 & 600 & $10 / 3 / 69$ & $10 / 16 / 69$ & 13 & 90 & 25 & 32 & 11 & 2 & 19 & 10 \\
\hline $39 \mathrm{~A}$ & 30,040 & 30,811 & 771 & $12 / 16 / 69$ & $1 / 10 / 70$ & 25 & 91 & 25 & 32 & 10 & 2 & 21 & 8 \\
\hline $39 B$ & 30,811 & 30,996 & 185 & $1 / 14 / 70$ & $1 / 19 / 70$ & 5 & 90 & 23 & 30 & 10 & 2 & 24 & 8 \\
\hline $39 \mathrm{C}$ & 30,996 & 31,411 & 415 & $1 / 19 / 70$ & $1 / 28 / 70$ & 9 & 90 & 23 & 30 & 10 & 2 & 24 & 8 \\
\hline $40 \mathrm{~A}$ & 31,411 & 31,854 & 443 & $1 / 31 / 70$ & $2 / 10 / 70$ & 10 & 91 & 26 & 27 & 10 & 2 & 25 & 10 \\
\hline $40 B$ & 31,854 & 32,778 & 924 & $2 / 10 / 70$ & $3 / 1 / 70$ & 19 & 91 & 26 & 27 & 10 & 2 & 25 & 10 \\
\hline $41 \mathrm{~A}$ & 32,778 & 33,739 & 961 & $3 / 5 / 70$ & $3 / 25 / 70$ & 20 & 91 & 26 & 28 & 10 & 2 & 23 & 9 \\
\hline $41 B$ & 33,739 & 33,896 & 157 & $3 / 27 / 70$ & $4 / 1 / 70$ & 5 & 91 & 26 & 28 & 10 & 2 & 23 & 10 \\
\hline 42 & 33,896 & 35,240 & 1,344 & $4 / 18 / 70$ & $5 / 18 / 70$ & 30 & 91 & 26 & 26 & 10 & 2 & 26 & 11 \\
\hline
\end{tabular}


EBR-II Power-run Information: Runs 1-50 (contd)

\begin{tabular}{|c|c|c|c|c|c|c|c|c|c|c|c|c|c|}
\hline \multirow{2}{*}{$\begin{array}{l}\text { Run } \\
\text { No. }\end{array}$} & \multicolumn{3}{|c|}{ Megawatt-Days } & \multicolumn{3}{|c|}{ Date } & \multirow{2}{*}{$\begin{array}{l}\text { Core } \\
\text { Size }\end{array}$} & \multicolumn{4}{|c|}{ Subassembly Type } & \multicolumn{2}{|c|}{ Experimentals } \\
\hline & Start & Stop & Incr. & Start & Stop & Days & & B & $\mathrm{C}$ & L & $S$ & Rows $1-6$ & Row 7 \\
\hline 43 & 35,240 & 36,590 & 1,350 & $5 / 26 / 70$ & $6 / 23 / 70$ & 28 & 91 & 25 & 29 & 10 & 2 & 24 & 10 \\
\hline $44 \mathrm{~A}$ & 36,590 & 37,490 & 900 & $6 / 26 / 70$ & $7 / 15 / 70$ & 19 & 91 & 24 & 30 & 10 & 2 & 24 & 9 \\
\hline $44 B$ & 37,490 & 37,941 & 451 & $7 / 25 / 70$ & $8 / 4 / 70$ & 10 & 91 & 24 & 32 & 10 & 2 & 22 & 9 \\
\hline $45 \mathrm{~A}$ & 37,941 & 38,586 & 645 & $8 / 9 / 70$ & $8 / 23 / 70$ & 14 & 90 & 23 & 35 & 10 & 2 & 17 & 6 \\
\hline $45 B$ & 38,586 & 39,291 & 705 & $8 / 26 / 70$ & $9 / 10 / 70$ & 15 & 91 & 24 & 35 & 10 & 2 & 17 & 5 \\
\hline $46 \mathrm{~A}$ & 39,291 & 40,042 & 751 & $9 / 20 / 70$ & $10 / 6 / 70$ & 16 & 88 & 26 & 39 & 10 & 2 & 7 & 11 \\
\hline $46 \mathrm{~B}$ & 40,042 & 40,841 & 799 & $10 / 8 / 70$ & $10 / 22 / 70$ & 14 & 88 & 26 & 39 & 11 & 2 & 9 & 10 \\
\hline $47 \mathrm{~A}$ & 40,841 & 41,816 & 975 & $10 / 28 / 70$ & $11 / 15 / 70$ & 18 & 89 & 23 & 38 & 10 & 2 & 13 & 11 \\
\hline $47 B$ & 41,816 & 42,321 & 505 & $3 / 2 / 71$ & $3 / 13 / 71$ & 11 & 91 & 22 & 37 & 10 & 2 & 19 & 15 \\
\hline $48 \mathrm{~A}$ & 42,321 & 42,340 & 19 & $3 / 31 / 71$ & $4 / 2 / 71$ & 3 & 88 & 20 & 38 & 9 & 2 & 16 & 13 \\
\hline $48 \mathrm{~B}$ & 42,340 & 42,400 & 60 & $4 / 3 / 71$ & $4 / 4 / 71$ & 1 & 88 & 20 & 38 & 9 & 2 & 16 & 13 \\
\hline $48 \mathrm{C}$ & 42,400 & 43,126 & 726 & $4 / 6 / 71$ & $4 / 20 / 71$ & 14 & 91 & 21 & 35 & 9 & 2 & 20 & 9 \\
\hline 48D & 43,126 & 43,776 & 650 & $4 / 22 / 71$ & $5 / 4 / 71$ & 12 & 91 & 16 & 36 & 9 & 2 & 25 & 9 \\
\hline
\end{tabular}


EBR-II Power-run Information: Runs 1-50 (contd)

\begin{tabular}{|c|c|c|c|c|c|c|c|c|c|c|c|c|c|}
\hline \multirow{2}{*}{$\begin{array}{l}\text { Run } \\
\text { No. }\end{array}$} & \multicolumn{3}{|c|}{ Megawatt-Days } & \multicolumn{3}{|c|}{ Date } & \multirow{2}{*}{$\begin{array}{l}\text { Core } \\
\text { Size }\end{array}$} & \multicolumn{4}{|c|}{ Subassemb1y Type } & \multicolumn{2}{|c|}{ Experimentals } \\
\hline & Start & Stop & Incr. & Start & Stop & Days & & $B$ & $\mathrm{C}$ & $\mathrm{L}$ & $\mathrm{S}$ & Rows $1-6$ & Row 7 \\
\hline $48 \mathrm{E}$ & 43,776 & 43,788 & 12 & $5 / 6 / 71$ & $5 / 7 / 71$ & $12.5 \mathrm{hr}$ & 91 & 16 & 36 & 9 & 2 & 26 & 9 \\
\hline $49 \mathrm{~A}$ & 43,788 & 43,852 & 64 & $5 / 18 / 71$ & $5 / 19 / 71$ & 1 & 91 & 16 & 35 & 9 & 2 & 27 & 14 \\
\hline $49 \mathrm{~B}$ & 43,852 & 44,442 & 590 & $5 / 20 / 71$ & $5 / 31 / 71$ & 11 & 91 & 16 & 35 & 9 & 2 & 27 & 14 \\
\hline $49 C$ & 44,442 & 44,716 & 274 & $6 / 3 / 71$ & $6 / 8 / 71$ & 5 & 91 & 16 & 36 & 9 & 2 & 26 & 14 \\
\hline 49D & 44,716 & 44,721 & 5 & $6 / 10 / 71$ & $6 / 10 / 71$ & $10 \mathrm{hr}$ & 90 & 19 & 37 & 9 & 2 & 21 & 13 \\
\hline $49 E$ & 44,721 & 44,734 & 13 & $6 / 14 / 71$ & $6 / 15 / 71$ & 1 & 89 & 21 & 38 & 9 & 2 & 17 & 12 \\
\hline $49 F$ & 44,734 & 45,299 & 565 & $6 / 20 / 71$ & $6 / 30 / 71$ & 10 & 89 & 20 & 42 & 9 & 2 & 14 & 11 \\
\hline $50 \mathrm{~A}$ & 45,299 & 45,361 & 62 & $7 / 4 / 71$ & $7 / 5 / 71$ & 1 & 90 & 20 & 39 & 9 & 2 & 18 & 10 \\
\hline $50 \mathrm{~B}$ & 45,361 & 45,372 & 11 & $7 / 6 / 71$ & $7 / 7 / 71$ & 1 & 89 & 20 & 40 & 9 & 2 & 16 & 13 \\
\hline $50 \mathrm{C}$ & 45,372 & 45,444 & 72 & $7 / 8 / 71$ & $7 / 11 / 71$ & 3 & 89 & 20 & 41 & 9 & 2 & 15 & 12 \\
\hline $50 \mathrm{D}$ & 45,444 & 45,514 & 70 & $7 / 11 / 71$ & $7 / 13 / 71$ & 2 & 89 & 20 & 41 & 9 & 2 & 15 & 11 \\
\hline $50 \mathrm{E}$ & 45,514 & 45,540 & 26 & $7 / 13 / 71$ & $7 / 14 / 71$ & 1 & 89 & 20 & 40 & 9 & 2 & 16 & 10 \\
\hline $50 F$ & 45,540 & 46,317 & 777 & $7 / 16 / 71$ & $7 / 29 / 71$ & 13 & 89 & 19 & 40 & 9 & 2 & 17 & 13 \\
\hline
\end{tabular}


EBR-II Power-run Information: Runs 1-50 (contd)

\begin{tabular}{|c|c|c|c|c|c|c|c|c|c|c|c|c|c|}
\hline \multirow{2}{*}{$\begin{array}{l}\text { Run } \\
\text { No. }\end{array}$} & \multicolumn{3}{|c|}{ Megawatt-Days } & \multicolumn{3}{|c|}{ Date } & \multirow{2}{*}{$\begin{array}{l}\text { Core } \\
\text { Size }\end{array}$} & \multicolumn{4}{|c|}{ Subassembly Type } & \multicolumn{2}{|c|}{ Experimentals } \\
\hline & Start & Stop & Incr. & Start & Stop & Days & & B & $\mathrm{C}$ & $\mathrm{L}$ & S & Rows $1-6$ & Row 7 \\
\hline $50 \mathrm{G}$ & 46,317 & 46,317 & 0 & $8 / 3 / 71$ & $8 / 3 / 71$ & $1 \mathrm{hr}$ & 90 & 16 & 33 & 9 & 2 & 21 & 13 \\
\hline $50 \mathrm{H}$ & 46,317 & 46,863 & 546 & $8 / 6 / 71$ & $8 / 15 / 71$ & 9 & 90 & 17 & 37 & 9 & 2 & 23 & 13 \\
\hline & & & & & & & & & & & & & \\
\hline & & & & & & & & & & & & & \\
\hline & & & & & & & & & & & & & \\
\hline & & & & & & & & & & & & & \\
\hline & & & & & & & & & & & & & \\
\hline & & & & & & & & & & & & & \\
\hline & & & & & & & & & & & & & \\
\hline & & & & & & & & & & & & & \\
\hline & & & & & & & & & & & & & \\
\hline & & & & & & & & & & & & & \\
\hline & & & & & & & & & & & & & \\
\hline
\end{tabular}

